\title{
HIGHER ORDER EXPANSIONS FOR THE PROBABILISTIC LOCAL CAUCHY THEORY OF THE CUBIC NONLINEAR SCHRÖDINGER EQUATION ON $\mathbb{R}^{3}$
}

\author{
ÁRPÁD BÉNYI, TADAHIRO OH, AND OANA POCOVNICU
}

\begin{abstract}
We consider the cubic nonlinear Schrödinger equation (NLS) on $\mathbb{R}^{3}$ with randomized initial data. In particular, we study an iterative approach based on a partial power series expansion in terms of the random initial data. By performing a fixed point argument around the second order expansion, we improve the regularity threshold for almost sure local well-posedness from our previous work. We further investigate a limitation of this iterative procedure. Finally, we introduce an alternative iterative approach, based on a modified expansion of arbitrary length, and prove almost sure local well-posedness of the cubic NLS in an almost optimal regularity range with respect to the original iterative approach based on a power series expansion.
\end{abstract}

\section{Contents}

1. Introduction

1.1. Nonlinear Schrödinger equation

1.2. Improved almost sure local well-posedness

1.3. Partial power series expansion and the associated critical regularity

2. Strichartz estimates and function spaces

2.1. Probabilistic Strichartz estimates

2.2. Function spaces and their properties

3. On the second order term $z_{3}$

4. On the higher order terms

4.1. On the third order term $z_{5}$

4.2. On the fourth order term $z_{7}$

4.3. On the "unbalanced" higher order terms $\zeta_{2 k-1}$

5. Proof of Theorem 1.3

6. Proof of Theorem 1.8

Appendix A. On the deterministic non-smoothing of the Duhamel integral operator

References

Received by the editors February 10, 2018, and, in revised form, August 20, 2018.

2010 Mathematics Subject Classification. Primary 35Q55.

Key words and phrases. Nonlinear Schrödinger equation, almost sure well-posedness, power series expansion.

This research was partially supported by Research in Groups at International Centre for Mathematical Sciences, Edinburgh, United Kingdom.

The first author was partially supported by a grant from the Simons Foundation (No. 246024).

The second author was supported by the European Research Council (grant no. 637995 "ProbDynDispEq"). 


\section{INTRODUCTION}

1.1. Nonlinear Schrödinger equation. We consider the Cauchy problem of the cubic nonlinear Schrödinger equation (NLS) on $\mathbb{R}^{3}[$

$$
\left\{\begin{array}{l}
i \partial_{t} u+\Delta u=|u|^{2} u, \\
\left.u\right|_{t=0}=u_{0} \in H^{s}\left(\mathbb{R}^{3}\right),
\end{array} \quad(t, x) \in \mathbb{R} \times \mathbb{R}^{3} .\right.
$$

The cubic NLS (1.1) has been studied extensively from both the theoretical and applied points of view. In this paper, we continue our study in [1,2] and further investigate the probabilistic well-posedness of (1.1) with random and rough initial data.

Recall that the equation (1.1) is scaling critical in $\dot{H}^{\frac{1}{2}}\left(\mathbb{R}^{3}\right)$ in the sense that the scaling symmetry

$$
u(t, x) \longmapsto \lambda u\left(\lambda^{2} t, \lambda x\right)
$$

preserves the homogeneous $\dot{H}^{\frac{1}{2}}$-norm (when it is applied to functions only of $x$ ). It is known that the Cauchy problem (1.1) is locally well-posed in $H^{s}\left(\mathbb{R}^{3}\right)$ for $s \geq \frac{1}{2}$ [10. See also [13, 18,26,29] for partial2 ${ }^{2}$ global well-posedness and scattering results. On the other hand, (1.1) is known to be ill-posed in $H^{s}\left(\mathbb{R}^{3}\right)$ for $s<$ $\frac{1}{2}$ [12]. In [2], we studied the probabilistic well-posedness property of (1.1) below the scaling critical regularity $s_{\text {crit }}=\frac{1}{2}$ under a suitable randomization of initial data; see (1.3) below. In particular, we proved that (1.1) is almost surely locally well-posed in $H^{s}\left(\mathbb{R}^{3}\right), s>\frac{1}{4}$. Our main goal in this paper is to introduce an iterative procedure to improve this regularity threshold for almost sure local well-posedness. Furthermore, we study a critical regularity associated with this iterative procedure. By introducing a modified iterative approach, we then prove almost sure local wellposedness of (1.1) in an almost optimal range with respect to the original iterative procedure (Theorem 1.8). Beyond the concrete results in this paper, we believe that the iterative procedures based on (modified) partial power series expansions are themselves of interest for further development in the well-posedness theory of dispersive equations with random initial data and/or random forcing.

Such a probabilistic construction of solutions to dispersive PDEs first appeared in the works by McKean [34 and Bourgain [5] in the study of invariant Gibbs measures for the cubic NLS on $\mathbb{T}^{d}, d=1,2$. In particular, they established almost sure local well-posedness with respect to particular random initial data, basically corresponding to the Brownian motion. (These local-in-time solutions were then extended globally in time by invariance of the Gibbs measures. In the following, however, we restrict our attention to local-in-time solutions.) In [8], Burq-Tzvetkov further elaborated this idea and considered a randomization for any rough initial condition via the Fourier series expansion. More precisely, they studied the cubic nonlinear wave equation (NLW) on a three-dimensional compact Riemannian manifold below the scaling critical regularity. By introducing a randomization via the multiplication of the Fourier coefficients by independent random variables, they

\footnotetext{
${ }^{1}$ Our discussion in this paper can be easily adapted to the cubic NLS on $\mathbb{R}^{d}$ (and to other nonlinear dispersive PDEs). For the sake of concreteness, however, we only consider the $d=3$ case in the following. See also the comment after Theorem 1.3 for our particular interest in the three-dimensional problem.

${ }^{2}$ Namely, either for smoother initial data or under an extra hypothesis.
} 
established almost sure local well-posedness below the critical regularity. Such randomization via the Fourier series expansion is natural on compact domains [15, 35. and more generally in situations where the associated elliptic operators have discrete spectra [45,47]. See [2,3] for more references therein.

In the following, we go over a randomization suitable for our problem on $\mathbb{R}^{3}$. Recall the Wiener decomposition of the frequency space [48]:

$$
\mathbb{R}_{\xi}^{3}=\bigcup_{n \in \mathbb{Z}^{3}}\left(n+\left(-\frac{1}{2}, \frac{1}{2}\right]^{3}\right) .
$$

We employ a randomization adapted to this Wiener decomposition. Let $\psi \in \mathcal{S}\left(\mathbb{R}^{3}\right)$ satisfy

$$
\operatorname{supp} \psi \subset[-1,1]^{3} \quad \text { and } \quad \sum_{n \in \mathbb{Z}^{3}} \psi(\xi-n)=1 \quad \text { for any } \xi \in \mathbb{R}^{3} .
$$

Then, given a function $\phi$ on $\mathbb{R}^{3}$, we have

$$
\phi=\sum_{n \in \mathbb{Z}^{3}} \psi(D-n) \phi
$$

We define the Wiener randomization $\phi^{\omega}$ of $\phi$ by

$$
\phi^{\omega}:=\sum_{n \in \mathbb{Z}^{3}} g_{n}(\omega) \psi(D-n) \phi,
$$

where $\left\{g_{n}\right\}_{n \in \mathbb{Z}^{3}}$ is a sequence of independent mean-zero complex-valued random variables on a probability space $(\Omega, \mathcal{F}, P)$. The randomization (1.3) based on the Wiener decomposition of the frequency space is natural in view of time-frequency analysis; see [1] for a further discussion. Almost simultaneously with [1, LührmannMendelson [32] also considered a randomization of the form (1.3) (with cubes being substituted by appropriately localized balls) in the study of NLW on $\mathbb{R}^{3}$. For a similar randomization used in the study of the Navier-Stokes equations, see also the work of Zhang and Fang [50, preceding [1, 32. In [50], the decomposition of the frequency space is not explicitly provided, but their randomization certainly includes randomization based on the decomposition of the frequency space by unit cubes or balls.

In the following, we assume that the probability distribution $\mu_{n}$ of $g_{n}$ satisfies the exponential moment bound

$$
\int_{\mathbb{R}^{2}} e^{\kappa \cdot x} d \mu_{n}(x) \leq e^{c|\kappa|^{2}}
$$

for all $\kappa \in \mathbb{R}^{2}$ and $n \in \mathbb{Z}^{3}$. This condition is satisfied by the standard complexvalued Gaussian random variables and the uniform distribution on the circle in the complex plane.

We now recall the almost sure local well-posedness result from [2] which is of interest to us 3

Theorem A. Let $\frac{1}{4}<s<\frac{1}{2}$. Given $\phi \in H^{s}\left(\mathbb{R}^{3}\right)$, let $\phi^{\omega}$ be its Wiener randomization defined in (1.3). Then, the Cauchy problem (1.1) is almost surely locally well-posed with respect to the random initial data $\phi^{\omega}$. More precisely, there exists

\footnotetext{
${ }^{3}$ For simplicity, we only consider positive times. By the time reversibility of the equation, the same analysis applies to negative times.
} 
a set $\Sigma=\Sigma(\phi) \subset \Omega$ with $P(\Sigma)=1$ such that, for any $\omega \in \Sigma$, there exists a unique function $u=u^{\omega}$ in the class

$$
S(t) \phi^{\omega}+X^{\frac{1}{2}}([0, T]) \subset S(t) \phi^{\omega}+C\left([0, T] ; H^{\frac{1}{2}}\left(\mathbb{R}^{3}\right)\right) \subset C\left([0, T] ; H^{s}\left(\mathbb{R}^{3}\right)\right)
$$

with $T=T(\phi, \omega)>0$ such that $u$ is a solution to (1.1) on $[0, T]$.

Here, $S(t):=e^{i t \Delta}$ denotes the linear Schrödinger operator, and the function space $X^{\frac{1}{2}}([0, T]) \subset C\left([0, T] ; H^{\frac{1}{2}}\left(\mathbb{R}^{3}\right)\right)$ is defined in Section 2 below. Note that the uniqueness statement of Theorem $\mathrm{A}$ in the class

$$
S(t) \phi^{\omega}+X^{\frac{1}{2}}([0, T])
$$

is to be interpreted as follows: by setting $v=u-S(t) \phi^{\omega}$ (see (1.7) below), uniqueness for the residual term $v$ holds in $X^{\frac{1}{2}}([0, T])$. See also Remark 1.1 below.

Recall that while the Wiener randomization (1.3) does not improve differentiability, it improves integrability (see Lemma 4 in [1]). See [8, 27, 42 for the corresponding statements in the context of the random Fourier series. The main idea for proving Theorem $\mathrm{A}$ is to exploit this gain of integrability. More precisely, let

$$
z_{1}(t):=S(t) \phi^{\omega}
$$

denote the random linear solution with $\phi^{\omega}$ as initial data and write

$$
u=z_{1}+v \text {. }
$$

Then, we see that $v:=u-z_{1}$ satisfies the following perturbed NLS:

$$
\left\{\begin{array}{l}
i \partial_{t} v+\Delta v=\left|v+z_{1}\right|^{2}\left(v+z_{1}\right), \\
\left.v\right|_{t=0}=0,
\end{array}\right.
$$

where $z_{1}$ is viewed as a given (random) source term. The main point is that the gain of space-time integrability of the random linear solution $z_{1}$ (Lemma 2.1) makes this problem subcritical $\Delta^{4}$ and hence we can solve it by a standard fixed point argument. Over the last several years, there have been many results on probabilistic wellposedness of nonlinear dispersive PDEs, using this change of viewpoint 5 See, for example, 1, 2, 5, 7, 9, 15, 25, 30, 32, 35, 37, 38, 43. In 2], we studied the Duhamel formulation for (1.8),

$$
v(t)=-i \int_{0}^{t} S\left(t-t^{\prime}\right)\left|v+z_{1}\right|^{2}\left(v+z_{1}\right)\left(t^{\prime}\right) d t^{\prime},
$$

by carrying out case-by-case analysis on terms of the form $v \bar{v} v, v \bar{v} z_{1}, v \bar{z}_{1} z_{1}$, etc., in $X^{\frac{1}{2}}([0, T])$. The main tools were (i) the gain of space-time integrability of $z_{1}$ and (ii) the bilinear refinement of the Strichartz estimate (Lemma 2.7). This yields Theorem $\mathrm{A}$,

\footnotetext{
${ }^{4}$ The scaling critical Sobolev regularity $s_{\text {crit }}=\frac{1}{2}$ for 11.1 is defined by the fact that the $\dot{H}^{\frac{1}{2}}$ norm remains invariant under the scaling symmetry (1.2). Given $1 \leq r \leq \infty$, we can also define the scaling critical Sobolev regularity $s_{\text {crit }}(r)$ in terms of the $L^{r}$-based Sobolev space $\dot{W}^{s, r}$. A direct computation gives $s_{\text {crit }}(r)=\frac{3}{r}-1(\rightarrow-1$ as $r \rightarrow \infty)$. In particular, the gain of integrability of the random linear solution $z_{1}$ stated in Lemma 2.1 implies that $z_{1}$ in (1.8) gives rise only to a subcritical perturbation. See [3] for a further discussion on this issue.

Note, however, that if we consider a non-zero initial condition for $v$, then the critical nature of the equation comes into play through the initial condition. See Remark 1.4 This plays an important role in studying the global-in-time behavior of solutions. See, for example, 37.

${ }^{5}$ In the field of stochastic parabolic PDEs, this change of viewpoint and solving the fixed point problem for the residual term $v$ is called the Da Prato-Debussche trick [16] 17.
} 
By examining the case-by-case analysis in [2], we see that the regularity restriction $s>\frac{1}{4}$ in Theorem $\mathrm{A}$ comes from the cubic interaction of the random linear solution:

$$
z_{3}(t):=-i \int_{0}^{t} S\left(t-t^{\prime}\right)\left|z_{1}\right|^{2} z_{1}\left(t^{\prime}\right) d t^{\prime} .
$$

See Proposition 1.2 below. In the following, we discuss an iterative approach to lower this regularity threshold by studying further expansions in terms of the random linear solution $z_{1}$. We will also discuss the limitation of this iterative procedure.

Remark 1.1.

(i) The proof of Theorem $\mathrm{A}$, presented in 2, is based on a standard contraction argument for the residual term $v=u-S(t) \phi^{\omega}$ in a ball $B$ of radius $O(1)$ in $X^{\frac{1}{2}}([0, T])$. As such, the argument in [2] only yields uniqueness of $v$ in the ball $B$. Noting that $v \in C\left([0, T] ; H^{\frac{1}{2}}\left(\mathbb{R}^{3}\right)\right) \cap X^{\frac{1}{2}}([0, T]) 6$ it follows from Lemma A.8 in [2] that the $X^{\frac{1}{2}}([0, t])$-norm of $v$ is continuous in $t \in[0, T]$. Then, by possibly shrinking the local existence time $T>0$, we can easily upgrade the uniqueness of $v$ in the ball $B$ to uniqueness of $v$ in the entire $X^{\frac{1}{2}}([0, T])$. Namely, uniqueness of $u$ holds in the class (1.5).

(ii) With the exponential moment assumption (1.4), the proof of Theorem A, presented in [2, allows us to conclude that the set $\Sigma$ of full probability in Theorem A has the following decomposition:

$$
\Sigma=\bigcup_{0<T \ll 1} \Sigma_{T}
$$

such that

(a) there exist $c, C>0$ and $C_{0}=C_{0}\left(\|\phi\|_{H^{s}}\right)>0$ such that

$$
P\left(\Sigma_{T}^{c}\right)<C \exp \left(-\frac{C_{0}\left(\|\phi\|_{H^{s}}\right)}{T^{c}}\right)
$$

for each $0<T \ll 1$,

(b) for each $\omega \in \Sigma_{T} \subset \Sigma, 0<T \ll 1$, the function $u=u^{\omega}$ constructed in Theorem $\mathrm{A}$ is a solution to (1.1) on $[0, T]$.

See the statement of Theorem 1.1 in [2]. A similar decomposition of the set $\Sigma$ of full probability applies to Theorems 1.3 and 1.8 below. We, however, do not state it in an explicit manner in the following.

1.2. Improved almost sure local well-posedness. Let us first state the following proposition on the regularity property of the cubic term $z_{3}$ defined above.

Proposition 1.2. Given $0 \leq s<1$, let $\phi^{\omega}$ be the Wiener randomization of $\phi \in$ $H^{s}\left(\mathbb{R}^{3}\right)$ defined in (1.3) and set $z_{1}=S(t) \phi^{\omega}$.

(i) For any $\sigma<2 s$, we have

$$
z_{3} \in X_{\mathrm{loc}}^{\sigma} \subset C\left(\mathbb{R} ; H^{\sigma}\left(\mathbb{R}^{3}\right)\right)
$$

\footnotetext{
${ }^{6} \mathrm{By}$ convention, our definition of the $X^{s}([0, T])$-space already assumes that functions in $X^{s}([0, T])$ belong to $C\left([0, T] ; H^{s}\left(\mathbb{R}^{3}\right)\right)$. See Definition 2.4 below.
} 
almost surely. More precisely, there exist an almost surely finite constant $C\left(\omega,\|\phi\|_{H^{s}}\right)>0$ and $\theta>0$ such that

$$
\left\|z_{3}\right\|_{X^{\sigma}([0, T])}=\left\|\int_{0}^{t} S\left(t-t^{\prime}\right)\left|z_{1}\right|^{2} z_{1}\left(t^{\prime}\right) d t^{\prime}\right\|_{X^{\sigma}([0, T])} \leq T^{\theta} C\left(\omega,\|\phi\|_{H^{s}}\right)
$$

for any $T>0$. In particular, by taking $\sigma=2 s-\varepsilon$ for small $\varepsilon>0$, (1.11) shows that the second order term $z_{3}$ is smoother $($ by $s-\varepsilon)$ than the first order term $z_{1}$, provided $s>0$.

(ii) When $s=0$, there is no smoothing in the second order term $z_{3}$ in general; there exists $\phi \in L^{2}\left(\mathbb{R}^{3}\right)$ such that the estimate (1.11) with $\sigma=\varepsilon>0$ fails for any $\varepsilon>0$.

In [2], we already proved (1.11) when $\sigma=\frac{1}{2}$ and $s>\frac{1}{4}$, giving the regularity restriction in Theorem A. See Section 3 for the proof of Proposition 1.2 for a general value of $\sigma$. In the proof of Theorem $\mathrm{A}$, in order to carry out the case-by-case analysis for (1.9), we need to have $z_{3} \in X_{\text {loc }}^{\frac{1}{2}}$ (where we have a deterministic local well-posedness for (1.1)). In view of Proposition 1.2, this imposes the regularity restriction $s>\frac{1}{4}$. Note, however, that even when $s \leq \frac{1}{4}, z_{3}$ is still a well defined space-time function of spatial regularity $2 s-<\frac{1}{2}$. This motivates us to consider the second order expansion

$$
u=z_{1}+z_{3}+v
$$

and remove the second order interaction $z_{1} \bar{z}_{1} z_{1}$. Indeed, the residual term $v:=$ $u-z_{1}-z_{3}$ satisfies the following equation:

$$
\left\{\begin{array}{l}
i \partial_{t} v+\Delta v=\mathcal{N}\left(v+z_{1}+z_{3}\right)-\mathcal{N}\left(z_{1}\right), \\
\left.v\right|_{t=0}=0
\end{array}\right.
$$

where $\mathcal{N}(u)=|u|^{2} u$. In terms of the Duhamel formulation, we have

$$
v(t)=-i \int_{0}^{t} S\left(t-t^{\prime}\right)\left\{\mathcal{N}\left(v+z_{1}+z_{3}\right)-\mathcal{N}\left(z_{1}\right)\right\}\left(t^{\prime}\right) d t^{\prime} .
$$

Then, by studying the fixed point problem (1.14) for $v$, we have the following improved almost sure local well-posedness of (1.1).

Theorem 1.3. Given $\frac{1}{2} \leq \sigma \leq 1$, let $\frac{2}{5} \sigma<s<\frac{1}{2}$. Given $\phi \in H^{s}\left(\mathbb{R}^{3}\right)$, let $\phi^{\omega}$ be its Wiener randomization defined in (1.3). Then, the cubic NLS (1.1) on $\mathbb{R}^{3}$ is almost surely locally well-posed with respect to the random initial data $\phi^{\omega}$. More precisely, there exists a set $\Sigma=\Sigma(\phi, \sigma) \subset \Omega$ with $P(\Sigma)=1$ such that, for any $\omega \in \Sigma$, there exists a unique function $u=u^{\omega}$ in the class

$$
\begin{aligned}
z_{1}+z_{3}+X^{\sigma}([0, T]) & \subset z_{1}+z_{3}+C\left([0, T] ; H^{\sigma}\left(\mathbb{R}^{3}\right)\right) \\
& \subset C\left([0, T] ; H^{s}\left(\mathbb{R}^{3}\right)\right)
\end{aligned}
$$

with $T=T(\phi, \omega)>0$ such that $u$ is a solution to (1.1) on $[0, T]$.

\footnotetext{
${ }^{7}$ Here, we referred to $z_{3}$ as the second order term since it corresponds to the second order term appearing in the (formal) power series expansion of solutions to (1.1) in terms of the random initial data. For the same reason, we refer to $z_{5}$ in (1.19) and $z_{7}$ in (1.22) as the third and fourth order terms in the following. See Subsection 1.3 below.
} 
As in Theorem A the uniqueness of $u$ in the class $z_{1}+z_{3}+X^{\sigma}([0, T])$ is to be interpreted as uniqueness of the residual term $v=u-z_{1}-z_{3}$ in $X^{\sigma}([0, T])$. See also Remark 1.1

By taking $\sigma=\frac{1}{2}$, Theorem 1.3 states that (1.1) is almost surely locally well-posed in $H^{s}\left(\mathbb{R}^{3}\right)$, provided $s>\frac{1}{5}$. This in particular improves the almost sure local wellposedness in Theorem $\mathrm{A}$. On the other hand, by taking $\sigma=1$, Theorem 1.3 allows us to construct a solution $v=u-z_{1}-z_{3} \in X^{1}([0, T]) \subset C\left([0, T] ; H^{1}\left(\mathbb{R}^{3}\right)\right)$, provided that $s>\frac{2}{5}$. In particular, we can take random initial data below the scaling critical regularity $s_{\text {crit }}=\frac{1}{2}$, while we construct the residual part $v$ in $X^{1}([0, T])$. This opens up a possibility of studying the global-in-time behavior of $v$, using the (nonconserved) energy of $v$ :

$$
E(v)(t)=\frac{1}{2} \int_{\mathbb{R}^{3}}|\nabla v(t, x)|^{2} d x+\frac{1}{4} \int_{\mathbb{R}^{3}}|v(t, x)|^{4} d x
$$

with random initial data below the scaling critical regularity. We remark that by inspecting the argument in [2], a modification of (the proof of) Theorem A yields almost sure local well-posedness of (1.1) in the class

$$
S(t) \phi^{\omega}+X^{1}([0, T]) \subset S(t) \phi^{\omega}+C\left([0, T] ; H^{1}\left(\mathbb{R}^{3}\right)\right)
$$

only for $s>\frac{1}{2}$. (This restriction on $s$ can be easily seen by setting $\sigma=1$ in Proposition [1.2, ) In particular, Theorem $\mathrm{A}$ does not allow us to take random initial data below the scaling critical regularity $s_{\text {crit }}=\frac{1}{2}$ in studying the global-intime behavior of $v \in X^{1}([0, T])$, namely at the level of the energy $E(v)$. As our focus in this paper is the local-in-time analysis, we do not pursue further this issue on almost sure global well-posedness of (1.1) below the scaling critical regularity in this paper. We, however, point out two recent results [30, 37] on almost sure global well-posedness below the energy space for the defocusing energy-critical NLS in higher dimensions.

The main strategy for proving Theorem 1.3 is to study the fixed point problem (1.14) by carrying out case-by-case analysis on

$$
w_{1} \overline{w_{2}} w_{3} \text { for } w_{i}=v, z_{1} \text {, or } z_{3}, i=1,2,3 \text {, but not all } w_{i} \text { equal to } z_{1}
$$

in $N^{\sigma}([0, T])$, where the dual norm is defined by

$$
\|F\|_{N^{\sigma}([0, T])}=\left\|\int_{0}^{t} S\left(t-t^{\prime}\right) F\left(t^{\prime}\right) d t^{\prime}\right\|_{X^{\sigma}([0, T])} .
$$

Note that the number of cases has increased from the case-by-case analysis in the proof of Theorem $\mathrm{A}$, where we had $w_{i}=v$ or $z_{1}$. One of the main ingredients is the smoothing on $z_{3}$ stated in Proposition 1.2 above. Note, however, that in order to exploit this smoothing, we need to measure $z_{3}$ in the $X^{2 s-}([0, T])$-norm, which imposes a certain rigidity on the space-time integrability 8 In order to prove Theorem 1.3. we also need to exploit a gain of integrability on $z_{3}$. In Lemma 3.3, we use the dispersive estimate (see (3.10) below) and the gain of integrability on each $z_{1}$ of the three factors in (1.10) and show that $z_{3}$ also enjoys a gain of integrability by giving up some differentiability.

\footnotetext{
${ }^{8}$ Namely, we need to measure $z_{3}$ in $L_{t}^{q}\left([0, T] ; W^{2 s-, r}\left(\mathbb{R}^{3}\right)\right)$ for admissible pairs $(q, r)$. See Lemma 2.6
} 
Remark 1.4. Given $\phi \in H^{s}\left(\mathbb{R}^{3}\right)$, let $\phi^{\omega}$ be its Wiener randomization defined in (1.3). Given $v_{0} \in H^{\frac{1}{2}}\left(\mathbb{R}^{3}\right)$, we can also consider (1.1) with the random initial data of the form $u_{0}^{\omega}=v_{0}+\phi^{\omega}$ :

$$
\left\{\begin{array}{l}
i \partial_{t} u+\Delta u=|u|^{2} u, \\
\left.u\right|_{t=0}=v_{0}+\phi^{\omega} .
\end{array}\right.
$$

Then, by slightly modifying the proofs, we see that the analogues of Theorems A and 1.3 (with $\sigma=\frac{1}{2}$ ) also hold for (1.16). Namely, (1.16) is almost surely locally well-posed, provided $s>\frac{1}{5}$. This amounts to considering the following Cauchy problems:

$$
\left\{\begin{array}{l}
i \partial_{t} v+\Delta v=\left|v+z_{1}\right|^{2}\left(v+z_{1}\right), \\
\left.v\right|_{t=0}=v_{0}
\end{array}\right.
$$

when $\frac{1}{4}<s<\frac{1}{2}$ and

$$
\left\{\begin{array}{l}
i \partial_{t} v+\Delta v=\mathcal{N}\left(v+z_{1}+z_{3}\right)-\mathcal{N}\left(z_{1}\right), \\
\left.v\right|_{t=0}=v_{0}
\end{array}\right.
$$

when $\frac{1}{5}<s \leq \frac{1}{4}$. In this case, the critical nature of the problem appears through the $v \bar{v} v$ interaction in the case-by-case analysis (1.15) due to the deterministic (non-zero) initial data $v_{0}$ at the critical regularity. The required modification is straightforward and thus we omit details. See Proposition 6.3 in [2] and Lemma 6.2 in 37 . We point out that the discussion in the next subsection also applies to (1.16).

\section{Remark 1.5 .}

(i) Let $\mathcal{I}\left(u_{1}, u_{2}, u_{3}\right)$ denote the trilinear operator defined by

$$
\mathcal{I}\left(u_{1}, u_{2}, u_{3}\right)(t):=-i \int_{0}^{t} S\left(t-t^{\prime}\right) u_{1} \overline{u_{2}} u_{3}\left(t^{\prime}\right) d t^{\prime} .
$$

Then, for $\sigma>3 s-1$, there is no finite constant $C>0$ such that

$$
\left\|\mathcal{I}\left(u_{1}, u_{2}, u_{3}\right)\right\|_{X^{\sigma}([0,1])} \leq C \prod_{j=1}^{3}\left\|\phi_{j}\right\|_{H^{s}}
$$

for all $\phi_{j} \in H^{s}\left(\mathbb{R}^{3}\right)$, where $u_{j}=S(t) \phi_{j}$. See Appendix A In particular, this shows that when $s \leq \frac{1}{2}$, there is no deterministic smoothing for $\mathcal{I}$; i.e., (1.18) does not hold for $\sigma>s$.

(ii) The proof of Proposition 1.2 only exploits "the randomization at the linear level". Given $s \in \mathbb{R}$, let $\mathcal{R}^{s}$ denote the class of functions defined by

$$
\begin{aligned}
\mathcal{R}^{s}=\left\{u \text { on } \mathbb{R} \times \mathbb{R}^{3}:\right. & i \partial_{t} u+\Delta u=0 \\
& \text { and } \left.u \in L_{t, \text { loc }}^{q} W_{x}^{s, r}\left(\mathbb{R}^{3}\right) \text { for any } 2 \leq q, r<\infty\right\} .
\end{aligned}
$$

Under the regularity assumption $\sigma<2 s$ and $0 \leq s<1$, it follows from the proof of Proposition 1.2 that the left-hand side of (1.18) is finite for any $u_{1}, u_{2}, u_{3} \in \mathcal{R}^{s} 9$ In other words, the proof of Proposition 1.2 only uses the fact that the random linear solution $z_{1}=S(t) \phi^{\omega}$ belongs to $\mathcal{R}^{s}$ almost surely; see the probabilistic Strichartz estimate (Lemma 2.1). The multilinear random structure of $z_{3}$ in terms of the

\footnotetext{
${ }^{9}$ Here, we only need finiteness of the $A_{3}^{s}$-norm defined in (3.3) for each $u_{j}, j=1,2,3$. See Remark 3.2
} 
random linear solution $z_{1}$ yields further cancellation. See, for example, Lemma 3.6 in [15. Such extra cancellation seems to improve only space-time integrability, and we do not know how to use it to improve the regularity threshold (i.e., differentiability) at this point. A similar comment applies to the unbalanced higher order terms $\zeta_{2 k-1}$ (including $z_{5}$ below) studied in Proposition 1.7 below.

1.3. Partial power series expansion and the associated critical regularity. In this subsection, we discuss possible improvements over Theorem 1.3 by considering further expansions. For this purpose, we fix $\sigma=\frac{1}{2}$ in the following. By examining the proof of Theorem 1.3. we see that the regularity restriction $s>\frac{1}{5}$ comes from the following third order term:

$$
z_{5}(t):=-i \sum_{\substack{j_{1}+j_{2}+j_{3}=5 \\ j_{1}, j_{2}, j_{3} \in\{1,3\}}} \int_{0}^{t} S\left(t-t^{\prime}\right) z_{j_{1}} \overline{z_{j_{2}}} z_{j_{3}}\left(t^{\prime}\right) d t^{\prime} .
$$

Namely, we have $\left(j_{1}, j_{2}, j_{3}\right)=(1,1,3)$ up to permutations. In Lemma 4.1 we show that given $0<s<\frac{1}{2}$, we have $z_{5} \in X_{\mathrm{loc}}^{\frac{5}{2} s-}$. In particular, we have $z_{5} \in X_{\mathrm{loc}}^{\frac{1}{2}}$, provided $s>\frac{1}{5}$, yielding the regularity threshold in Theorem 1.3 .

A natural next step is to remove this non-desirable third order interaction

$$
\sum_{\substack{j_{1}+j_{2}+j_{3}=5 \\ j_{1}, j_{2}, j_{3} \in\{1,3\}}} z_{j_{1}} \overline{z_{2}} z_{j_{3}}
$$

in the case-by-case analysis in (1.15) by considering the following third order expansion:

$$
u=z_{1}+z_{3}+z_{5}+v .
$$

In this case, the residual term $v:=u-z_{1}-z_{3}-z_{5}$ satisfies the following equation:

$$
\left\{\begin{array}{l}
i \partial_{t} v+\Delta v=\mathcal{N}\left(v+z_{1}+z_{3}+z_{5}\right)-\sum_{\substack{j_{1}+j_{2}+j_{3} \in\{3,5\} \\
j_{1}, j_{2}, j_{3} \in\{1,3\}}} z_{j_{1}} \overline{z_{j_{2}}} z_{j_{3}}, \\
\left.v\right|_{t=0}=0 .
\end{array}\right.
$$

We expect that (1.21) is almost surely locally well-posed for $s>\frac{2}{11}$, which would be an improvement over Theorem [1.3. The proof will be once again based on case-by-case analysis:

$$
\begin{aligned}
w_{1} \overline{w_{2}} w_{3} & \text { for } w_{i}=v, z_{1}, z_{3} \text {, or } z_{5}, i=1,2,3, \text { such that } \\
& \text { it is not of the form } z_{j_{1}} \overline{z_{j_{2}}} z_{j_{3}} \text { with } j_{1}+j_{2}+j_{3} \in\{3,5\}
\end{aligned}
$$

in $N^{\frac{1}{2}}([0, T])$. Note the increasing number of combinations. In the following, however, we do not discuss details of this particular improvement over Theorem 1.3 , Instead, we consider further iterative steps and discuss a possible limitation of this procedure.

Remark 1.6. We point out that the expansions (1.7), (1.12), and (1.20) correspond to partial power series expansions of the first, second, and third orders 10 respectively, of a solution to (1.1) in terms of the random initial data. Then, by considering

\footnotetext{
${ }^{10} \mathrm{~A}$ (partial) power series expansion of a solution to (1.1) in terms of the random initial data can be expressed as a summation of certain multilinear operators over ternary trees. See, for example, [1],36]. Here, the term "order" in our context corresponds to "generation (of the associated trees) +1 " with the convention that the trivial tree consisting only of the root node is
} 
the associated equations (1.8), (1.13), and (1.21) for the residual term $v$, we are recasting the original problem (1.1) as a fixed point problem centered at the partial power series expansions of the first, second, and third orders, respectively.

By drawing an analogy to the previous steps, we expect that the worst contribution comes from the following fourth order terms:

$$
z_{7}(t):=-i \sum_{\substack{j_{1}+j_{2}+j_{3}=7 \\ j_{1}, j_{2}, j_{3} \in\{1,3,5\}}} \int_{0}^{t} S\left(t-t^{\prime}\right) z_{j_{1}} \overline{z_{j_{2}}} z_{j_{3}}\left(t^{\prime}\right) d t^{\prime} .
$$

There are basically two contributions to (1.22): $\left(j_{1}, j_{2}, j_{3}\right)=(1,3,3)$ or $(1,1,5)$ up to permutations. In Lemma 4.2, we show that the contribution from $\left(j_{1}, j_{2}, j_{3}\right)=$ $(1,1,5)$ is worse, being responsible for the expected regularity restriction $s>\frac{2}{11}$. In order to remove this term, we can consider the fourth order expansion

$$
u=z_{1}+z_{3}+z_{5}+z_{7}+v
$$

as in the previous steps and try to solve the following equation for the residual term $v=u-z_{1}-z_{3}-z_{5}-z_{7}$ :

$$
\left\{\begin{array}{l}
i \partial_{t} v+\Delta v=\mathcal{N}\left(v+z_{1}+z_{3}+z_{5}+z_{7}\right)-\sum_{\substack{j_{1}+j_{2}+j_{3} \in\{3,5,7\} \\
j_{1}, j_{2}, j_{3} \in\{1,3,5\}}} z_{j_{1}} \overline{z_{j_{2}}} z_{j_{3}}, \\
\left.v\right|_{t=0}=0 .
\end{array}\right.
$$

We can obviously iterate this argument and consider the following $k$ th order expansion:

$$
u=\sum_{\ell=1}^{k} z_{2 \ell-1}+v .
$$

In this case, we need to consider the following equation for the residual term $v:=$ $u-\sum_{\ell=1}^{k} z_{2 \ell-1}$ :

$$
\left\{\begin{array}{l}
i \partial_{t} v+\Delta v=\mathcal{N}\left(v+\sum_{\ell=1}^{k} z_{2 \ell-1}\right)-\sum_{\substack{j_{1}+j_{2}+j_{3} \in\{3,5, \ldots, 2 k-1\} \\
j_{1}, j_{2}, j_{3} \in\{1,3, \ldots, 2 k-3\}}} z_{j_{1}} \overline{z_{j_{2}}} z_{j_{3}}, \\
\left.v\right|_{t=0}=0
\end{array}\right.
$$

and hope to construct a solution $v \in X^{\frac{1}{2}}([0, T])$ by carrying out the following case-by-case analysis:

$$
\begin{aligned}
w_{1} \overline{w_{2}} w_{3}, & \text { for } w_{i}=v, z_{j}, j \in\{1,3, \ldots, 2 k-1\}, i=1,2,3, \text { such that } \\
& \text { it is not of the form } z_{j_{1}} \overline{z_{j_{2}}} z_{j_{3}} \text { with } j_{1}+j_{2}+j_{3} \in\{3,5, \ldots, 2 k-1\}
\end{aligned}
$$

of the zeroth generation. For example, the third order term $z_{5}$ in (1.19) appears as the summation over all the multilinear operators associated to the ternary trees of the third generation. In terms of the graphical representation in [36], we have

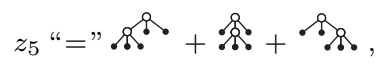

where "•" denotes the random linear solution $z_{1}=S(t) \phi^{\omega}$ and "॰" denotes the trilinear Duhamel integral operator $\mathcal{I}\left(u_{1}, u_{2}, u_{3}\right)$ defined in (1.17) with its three children as its arguments, $u_{1}, u_{2}$, and $u_{3}$. 
in $N^{\frac{1}{2}}([0, T])$. Then, a natural question to ask is, Does this iterative procedure work indefinitely, allowing us to arbitrarily lower the regularity threshold for almost sure local well-posedness for (1.1)? Or is there any limitation to it?

We now consider a "critical" regularity $s_{*}<\frac{1}{2}$ with respect to this iterative procedure for proving almost sure local well-posedness of (1.1). We simply define the critical regularity $s_{*}<\frac{1}{2}$ for this problem to be the infimum of the values of $s<\frac{1}{2}$ such that given any $\phi \in H^{s}\left(\mathbb{R}^{3}\right)$, the above iterative procedure1 shows that (1.1) is almost surely locally well-posed with respect to the Wiener randomization $\phi^{\omega}$ of $\phi$. This is an empirical notion of criticality; unlike the scaling criticality, we cannot a priori compute this critical regularity $s_{*}$. Moreover, our discussion will be based on the estimates on the stochastic multilinear terms (Propositions 1.2 and 1.7 below). In the following, we discuss a (possible) lower bound on $s_{*}$, presenting a limitation to our iterative procedure based on partial power series expansions.

Within the framework of the iterative procedure discussed above, a necessary condition for carrying out the case-by-case analysis (1.25) to study (1.24) in $X^{\frac{1}{2}}([0, T])$ is that the $(k+1)$ st order term $z_{2 k+1}=z_{2(k+1)-1}$ defined in a recursive manner,

$$
z_{2 k+1}(t):=-i \sum_{\substack{j_{1}+j_{2}+j_{3}=2 k+1 \\ j_{1}, j_{2}, j_{3} \in\{1,3, \ldots, 2 k-1\}}} \int_{0}^{t} S\left(t-t^{\prime}\right) z_{j_{1}} \overline{z_{j_{2}}} z_{j_{3}}\left(t^{\prime}\right) d t^{\prime}
$$

belongs to $X^{\frac{1}{2}}([0, T])$. By the nature of this iterative procedure, we may assume that the lower order terms $z_{2 \ell-1}, \ell \in\{1,3, \ldots, k\}$, belong to $X^{s_{\ell}}([0, T])$ for some $s_{\ell}<\frac{1}{2}$ but not in $X^{\frac{1}{2}}([0, T])$, since if any of the lower order terms, say $z_{2 \ell-1}$ for some $\ell \in\{1,3, \ldots, k\}$, were in $X^{\frac{1}{2}}([0, T])$, then we would have stopped the iterative procedure at the $(\ell-1)$ th step. As in the previous steps, we expect that $z_{2 k+1}$ is responsible for a regularity restriction at the $k$ th step of this iterative approach.

It could be a cumbersome task to study the regularity property of $z_{2 k+1}$ due to the increasing number of combinations for $z_{j_{1}} \overline{z_{j_{2}}} z_{j_{3}}$ satisfying $j_{1}+j_{2}+j_{3}=2 k+1$, $j_{1}, j_{2}, j_{3} \in\{1,3, \ldots, 2 k-1\}$. In the following, we instead study the regularity property of the $(k+1)$ st order term 12 of a particular form, corresponding to $\left(j_{1}, j_{2}, j_{3}\right)=(1,1,2 k-1)$ up to permutations in (1.26). Given an integer $k \geq 0$, define $\zeta_{2 k+1}$ by setting $\zeta_{1}:=z_{1}=S(t) \phi^{\omega}$ and

$$
\zeta_{2 k+1}(t):=-i \sum_{\substack{j_{1}+j_{2}+j_{3}=2 k+1 \\ j_{1}, j_{2}, j_{3} \in\{1,2 k-1\}}} \int_{0}^{t} S\left(t-t^{\prime}\right) \zeta_{j_{1}} \overline{\zeta_{j_{2}}} \zeta_{j_{3}}\left(t^{\prime}\right) d t^{\prime} .
$$

As mentioned above, there are only three terms in this sum: $\left(j_{1}, j_{2}, j_{3}\right)=(1,1,2 k-1)$ up to permutations. Hence, $\zeta_{2 k+1}$ consists of the "unbalanced" $13(2 k+1)$-linear terms appearing in the definition (1.26) of $z_{2 k+1}$. We claim that the $(k+1)$ st order term $\zeta_{2 k+1}$ is responsible for the regularity restriction at the $k$ th step of the iterative procedure. See Theorem 1.8 below.

\footnotetext{
${ }^{11}$ Namely, we solve (1.24) for $v \in X^{\frac{1}{2}}([0, T])$ with some finite (or infinite) number of steps.

${ }^{12}$ In fact, we study the $k$ th order term $\zeta_{2 k-1}$ in Proposition 1.7

${ }^{13} \mathrm{By}$ associating the $(2 k+1)$-linear terms appearing in the summation in (1.26) with ternary trees of the $k$ th generation as in [36], the summands in (1.27) correspond to the "unbalanced" trees of the $k$ th generation, where two of the three children of the root node are terminal.
} 
In anticipating the alternative expansion (1.33) below, let us study the regularity property of the unbalanced $k$ th order term $\zeta_{2 k-1}$ :

$$
\zeta_{2 k-1}(t):=-i \sum_{\substack{j_{1}+j_{2}+j_{3}=2 k-1 \\ j_{1}, j_{2}, j_{3} \in\{1,2 k-3\}}} \int_{0}^{t} S\left(t-t^{\prime}\right) \zeta_{j_{1}} \overline{\zeta_{j_{2}}} \zeta_{j_{3}}\left(t^{\prime}\right) d t^{\prime} .
$$

For $k=2,3,4$, we have (with appropriate restrictions on the range of $s$ )

$$
\zeta_{3}=z_{3} \in X_{\mathrm{loc}}^{2 s-}, \quad \zeta_{5}=z_{5} \in X_{\mathrm{loc}}^{\frac{5}{2} s-}, \quad \text { and } \quad \zeta_{7} \in X_{\mathrm{loc}}^{\frac{11}{4} s-} .
$$

See Proposition 1.2 and Lemmas 4.1 and 4.2 . In general, we have the following proposition.

Proposition 1.7. Define a sequence $\left\{\alpha_{k}\right\}_{k \in \mathbb{N}}$ of positive real numbers by the recursive relation

$$
\alpha_{k}=\frac{\alpha_{k-1}+3}{2}
$$

with $\alpha_{1}=1$. Given $0<s<\alpha_{k-1}^{-1}$, let $\phi^{\omega}$ be the Wiener randomization of $\phi \in$ $H^{s}\left(\mathbb{R}^{3}\right)$ defined in (1.3). Then, we have

$$
\zeta_{2 k-1} \in X_{\mathrm{loc}}^{\sigma}
$$

for $\sigma<\alpha_{k} \cdot s$, almost surely.

By solving the recursive relation (1.30), we have

$$
\alpha_{k}=2\left\{1-\left(\frac{1}{2}\right)^{k-1}\right\}+1
$$

and thus we have $\alpha_{2}=2, \alpha_{3}=\frac{5}{2}$, and $\alpha_{4}=\frac{11}{4}$. In particular, Proposition 1.7 agrees with (1.29). Moreover, since $\alpha_{k}$ is increasing and $\lim _{k \rightarrow \infty} \alpha_{k}=3$, the regularity restriction $s<\alpha_{k-1}^{-1}$ in Proposition 1.7 does not cause any issue since our main focus is to study the probabilistic local well-posedness of (1.1) in the range of $s$ that is not covered by Theorem 1.3. Namely, we may assume $s \leq \frac{1}{5}\left(<\frac{1}{3}\right)$ in the following.

In view of Propositions 1.2 and 1.7, one obvious lower bound for the critical regularity $s_{*}$ for this iterative procedure is given by $s_{0}:=0$ since there is no gain of regularity when $s=0$ (even in moving from $z_{1}$ to $z_{3}$ ). On the other hand, in order to prove almost sure local well-posedness of (1.1) by carrying out the case-by-case analysis (1.25) for the equation (1.24), we need to show that the $(k+1)$ st order term $\zeta_{2 k+1}$ belongs to $X^{\frac{1}{2}}([0, T])$. This gives rise to a regularity restriction $s_{k}:=\frac{1}{2 \alpha_{k+1}}$ at the $k$ th step of the iterative procedure. By taking $k \rightarrow \infty$, we obtain another "lower" bound $s_{\infty}:=\frac{1}{6}$ on this critical regularity $s_{*}$.

As mentioned above, the case-by-case analysis (1.25) for general $k \in \mathbb{N}$ may be a combinatorially overwhelming task due to (i) the number of the increasing combinations in (1.25) and (ii) the random multilinear terms $z_{j}, j \in\{3,5, \ldots, 2 k-1\}$, themselves having non-trivial combinatorial structures which makes it difficult to

\footnotetext{
${ }^{14}$ This "lower" bound is based on the upper bounds obtained in Propositions 1.2 and 1.7 In other words, if one can improve the bounds in Propositions 1.2 and 1.7 then one can lower the value of $s_{\infty}$. See Remark 1.10
} 
establish nonlinear estimates; see (1.26). In the following, we instead consider an alternative iterative procedure based on the expansion

$$
u=\sum_{\ell=1}^{k} \zeta_{2 \ell-1}+v
$$

in place of (1.23). This expansion allows us to prove the following almost sure local well-posedness of (1.1) for $s>s_{\infty}=\frac{1}{6}$.

Theorem 1.8. Let $\frac{1}{6}<s<\frac{1}{2}$. Given $\phi \in H^{s}\left(\mathbb{R}^{3}\right)$, let $\phi^{\omega}$ be its Wiener randomization defined in (1.3). Then, the cubic NLS (1.1) on $\mathbb{R}^{3}$ is almost surely locally well-posed with respect to the random initial data $\phi^{\omega}$. More precisely, there exists a set $\Sigma=\Sigma(\phi) \subset \Omega$ with $P(\Sigma)=1$ such that, for any $\omega \in \Sigma$, there exists a unique function $u=u^{\omega}$ in the class:

$$
\begin{aligned}
\zeta_{1}+\zeta_{3}+\cdots+\zeta_{2 k-1}+X^{\frac{1}{2}}([0, T]) & \subset \zeta_{1}+\zeta_{3}+\cdots+\zeta_{2 k-1}+C\left([0, T] ; H^{\frac{1}{2}}\left(\mathbb{R}^{3}\right)\right) \\
& \subset C\left([0, T] ; H^{s}\left(\mathbb{R}^{3}\right)\right)
\end{aligned}
$$

with $T=T(\phi, \omega)>0$ such that $u$ is a solution to (1.1) on $[0, T]$. Here, $k \in \mathbb{N}$ is a unique positive integer such that $\frac{1}{2 \alpha_{k+1}}<s \leq \frac{1}{2 \alpha_{k}}$.

As before, the uniqueness of $u$ in the class

$$
\zeta_{1}+\zeta_{3}+\cdots+\zeta_{2 k-1}+X^{\frac{1}{2}}([0, T])
$$

is to be interpreted as uniqueness of the residual term $v=u-\sum_{\ell=1}^{k} \zeta_{2 \ell-1}$ in $X^{\frac{1}{2}}([0, T])$. See also Remark 1.1

In view of the discussion above, Theorem 1.8 proves almost sure local wellposedness of (1.1) in an almost "optimal" 15 regularity range $s>s_{\infty}=\frac{1}{6}$ with respect to the original iterative procedure based on the partial power series expansion (1.23). The proof of Theorem 1.8 is analogous to that of Theorem 1.3. Given $\frac{1}{6}<s<\frac{1}{2}$, fix $k \in \mathbb{N}$ such that $\frac{1}{2 \alpha_{k+1}}<s \leq \frac{1}{2 \alpha_{k}} 16$ Write a solution $u$ as in (1.33). Note that as $s$ gets closer and closer to the critical value $s_{\infty}=\frac{1}{6}$, the expansion (1.33) gets arbitrarily long. In view of (1.28), the residual term $v:=u-\sum_{\ell=1}^{k} \zeta_{2 \ell-1}$ satisfies the following equation:

$$
\left\{\begin{array}{l}
i \partial_{t} v+\Delta v=\mathcal{N}\left(v+\sum_{\ell=1}^{k} \zeta_{2 \ell-1}\right)-\sum_{\ell=2}^{k} \sum_{\substack{j_{1}+j_{2}+j_{3}=2 \ell-1 \\
j_{1}, j_{2}, j_{3} \in\{1,2 \ell-3\}}} \zeta_{j_{1}} \overline{\zeta_{j_{2}}} \zeta_{j_{3}}, \\
\left.v\right|_{t=0}=0 .
\end{array}\right.
$$

\footnotetext{
${ }^{15}$ Once again, this is based on the estimates in Propositions 1.2 and 1.7 In particular, the "optimality" of the regularity threshold in Theorem 1.8 is with respect to Propositions 1.2 and 1.7 If one can improve the bounds in Propositions 1.2 and 1.7 then one can lower the regularity threshold in Theorem 1.8

${ }^{16} \mathrm{In}$ view of Proposition 1.7 the lower bound on $s$ guarantees that $\zeta_{2 k+1} \in X^{\frac{1}{2}}([0, T])$ almost surely, while the upper bound on $s$ states that we cannot use Proposition 1.7 to conclude $\zeta_{2 k-1} \in$ $X^{\frac{1}{2}}([0, T])$ almost surely.
} 
Hence, we need to carry out the following case-by-case analysis:

$$
\begin{aligned}
w_{1} \overline{w_{2}} w_{3}, & \text { for } w_{i}=v, \zeta_{j}, j \in\{1,3, \ldots, 2 k-1\}, i=1,2,3, \text { such that } \\
& \text { it is not of the form } \zeta_{j_{1}} \overline{\zeta_{j_{2}}} \zeta_{j_{3}} \text { with }\left(j_{1}, j_{2}, j_{3}\right)=(1,1,2 \ell-3) \\
& \text { (up to permutations) for } \ell=2,3, \ldots, k
\end{aligned}
$$

in $N^{\frac{1}{2}}([0, T])$. While Theorem 1.8 yields almost sure local well-posedness in an almost optimal range with respect to the original iterative procedure based on the partial power series expansion (1.23), the required analysis is much simpler than that required for the original iterative procedure based on the expansion (1.23). First, note that while the case-by-case analysis (1.25) based on the original iterative procedure involves combinatorially non-trivial $z_{2 k-1}$, the case-by-case analysis (1.35) only involves the unbalanced $k$ th order term $\zeta_{2 k-1}$ which has a much simpler structure than $z_{2 k-1}$. In particular, Proposition 1.7 shows that $\zeta_{2 k-1}, k \geq 3$, has a better regularity property than the second order term $\zeta_{3}=z_{3}$ in (1.10). In terms of space-time integrability, we show that $\zeta_{2 k-1}$ also enjoys a gain of integrability by giving up control on derivatives (Lemma 4.3). Finally, by inspecting the proof of Theorem 1.3 (see Lemma 4.2 and Proposition 5.1 below), we see that, except for $z_{j_{1}} \overline{z_{j_{2}}} z_{j_{3}}$ with $\left(j_{1}, j_{2}, j_{3}\right)=(1,1,3)$ up to permutations 17 we can bound all the terms $w_{1} \overline{w_{2}} w_{3}$ appearing in the case-by-case analysis (1.15) in $N^{\frac{1}{2}}([0, T])$. Hence, we can basically apply the result of the case-by-case analysis (1.15) to our problem at hand. More precisely, by rewriting the case-by-case analysis (1.35) as

$$
w_{i}=v, \quad \zeta_{1}=z_{1}, \quad \text { or } \quad \zeta_{j}, j \in\{3,5, \ldots, 2 k-1\}
$$

(with the restriction in (1.35) ) and using the fact that the terms $\zeta_{2 \ell-1}, \ell=3, \ldots, k$, behave better than $\zeta_{3}=z_{3}$, the proof of Theorem 1.3 (in particular, Proposition 5.1) can be used to control all the terms $w_{1} \overline{w_{2}} w_{3}$ in (1.35) except for

$$
\zeta_{j_{1}} \overline{\zeta_{j_{2}}} \zeta_{j_{3}} \text { with }\left(j_{1}, j_{2}, j_{3}\right)=(1,1,2 k-1)
$$

(up to permutations). Note that the contribution to (1.36) under the Duhamel integral is precisely given by $\zeta_{2 k+1}$ in (1.27). In particular, Proposition 1.7 with $\sigma=\frac{1}{2}$ yields the regularity restriction $\alpha_{k+1} \cdot s>\frac{1}{2}$, i.e., the lower bound $s>\frac{1}{2 \alpha_{k+1}}$ stated in Theorem 1.8. This allows us to construct a solution $v \in X^{\frac{1}{2}}([0, T])$ by the standard fixed point argument. See Section 6 for details.

We previously conjectured that the $(k+1)$ st order term $z_{2 k+1}$ in (1.26) would be responsible for a regularity restriction at the $k$ th step of the original iterative procedure. Combining Proposition 1.7 and Theorem 1.8, we confirmed this claim; the regularity restriction indeed comes only from the unbalanced $(k+1)$ st order term $\zeta_{2 k+1}$ in (1.27).

We conclude this introduction with several remarks.

Remark 1.9. Let $v_{0} \in H^{\frac{1}{2}}\left(\mathbb{R}^{3}\right)$. Then, by slightly modifying the proof of Theorem 1.8 based on the modified iterative approach (1.33), we can prove almost sure local well-posedness of the cubic NLS (1.16) with the random initial data of the form $v_{0}+\phi^{\omega}$ for the same range of $s$. See Remark 1.4 .

Remark 1.10. In this paper, we exploit randomness only at the linear level in estimating the stochastic terms. See Remarks 1.5 and 3.2. It may be possible to lower the regularity thresholds in Theorems 1.3 and 1.8 by exploiting randomness at

\footnotetext{
${ }^{17}$ Namely, the terms constituting the third order term $\zeta_{5}=z_{5}$ in (1.19).
} 
the multilinear level. We, however, do not pursue this direction in this paper since (i) our main purpose is to present the iterative procedures in their simplest forms and (ii) estimating the higher order stochastic terms by exploiting randomness at the multilinear level would require a significant amount of additional work, which would blur the main focus of this paper.

Remark 1.11. The ill-posedness result in [12] shows that the solution map

$$
\Phi: u_{0} \in H^{s}\left(\mathbb{R}^{3}\right) \longmapsto u \in C\left([-T, T] ; H^{s}\left(\mathbb{R}^{3}\right)\right)
$$

is not continuous for (1.1) when $s<\frac{1}{2}$. In proving Theorem A, we studied the perturbed NLS (1.8) for $v=u-z_{1}$. In particular, the proof shows that we can factorize the solution map for (1.1) a: 18

$$
\phi^{\omega} \in H^{s}\left(\mathbb{R}^{3}\right) \longmapsto z_{1} \stackrel{\Psi}{\longmapsto} v \in X^{\frac{1}{2}}([0, T]) \subset C\left([0, T] ; H^{\frac{1}{2}}\left(\mathbb{R}^{3}\right)\right),
$$

where the first map can be viewed as a universal lift map and the second map $\Psi$ is the solution map to (1.8), which is in fact continuous in $z_{1} \in S_{1}^{s}([0, T]) 19$

In the case of Theorem 1.3 (with $\sigma=\frac{1}{2}$ ), we have the following factorization of the solution map for (1.1):

$$
\phi^{\omega} \in H^{s}\left(\mathbb{R}^{3}\right) \longmapsto\left(z_{1}, z_{3}\right) \stackrel{\Psi}{\longmapsto} v \in X^{\frac{1}{2}}([0, T]) \subset C\left([0, T] ; H^{\frac{1}{2}}\left(\mathbb{R}^{3}\right)\right),
$$

where the second map $\Psi$ is the solution map to (1.13), which is continuous from $\left(z_{1}, z_{3}\right) \in S_{1}^{s}([0, T]) \times S_{3}^{2 s-}([0, T])$ to $v \in X^{\frac{1}{2}}([0, T])$.

In the case of Theorem 1.8, we create $k$ stochastic objects in the first step, where $k=k(s) \in \mathbb{N}:$

$$
\phi^{\omega} \in H^{s}\left(\mathbb{R}^{3}\right) \longmapsto\left(\zeta_{1}, \zeta_{3}, \ldots, \zeta_{2 k-1}\right) \stackrel{\Psi}{\longmapsto} v \in X^{\frac{1}{2}}([0, T]) \subset C\left([0, T] ; H^{\frac{1}{2}}\left(\mathbb{R}^{3}\right)\right) .
$$

Once again, the second map $\Psi$ (which is the solution map to (1.34) ) is continuous from $\left(\zeta_{1}, \zeta_{3}, \ldots, \zeta_{2 k-1}\right) \in S_{1}^{s}([0, T]) \times \prod_{\ell=2}^{k} S_{\ell}^{\alpha_{\ell} s^{-}}([0, T])$ to $v \in X^{\frac{1}{2}}([0, T])$. We point out an analogy between these factorizations (1.37), (1.38), and (1.39) of the ill-posed solution maps into

(i) the first step, involving stochastic analysis, and

(ii) the second step, where purely deterministic analysis is performed in constructing a continuous map $\Psi$,

and similar factorizations for studying rough differential equations via the rough path theory [19] and singular stochastic parabolic PDEs [21,23.

In the proof of Theorem 1.8, we could consider the following expansion of an infinite order:

$$
u=\sum_{\ell=1}^{\infty} \zeta_{2 \ell-1}+v .
$$

\footnotetext{
${ }^{18}$ Similarly, we can factorize the solution map for 1.16 as

$$
\left.u\right|_{t=0}=v_{0}+\phi^{\omega} \in H^{s}\left(\mathbb{R}^{3}\right) \longmapsto\left(v_{0}, z_{1}\right) \stackrel{\Psi}{\longmapsto} v \in X^{\frac{1}{2}}([0, T]) \subset C\left([0, T] ; H^{\frac{1}{2}}\left(\mathbb{R}^{3}\right)\right)
$$
}

such that the second map $\Psi$ is continuous in $\left(v_{0}, z_{1}\right) \in H^{\frac{1}{2}}\left(\mathbb{R}^{3}\right) \times S_{1}^{s}([0, T])$.

${ }^{19}$ Here, $S_{1}^{S}([0, T]) \subset C\left([0, T] ; H^{s}\left(\mathbb{R}^{3}\right)\right)$ denotes the intersection of suitable space-time function spaces of differentiability at most $s$. In the following, we use $S_{j}^{\sigma}([0, T])$ in a similar manner. 
This would allow us to present a single argument that works for all $\frac{1}{6}<s<\frac{1}{3}$. In this case, the residual part $v:=u-\sum_{\ell=1}^{\infty} \zeta_{2 \ell-1}$ satisfies

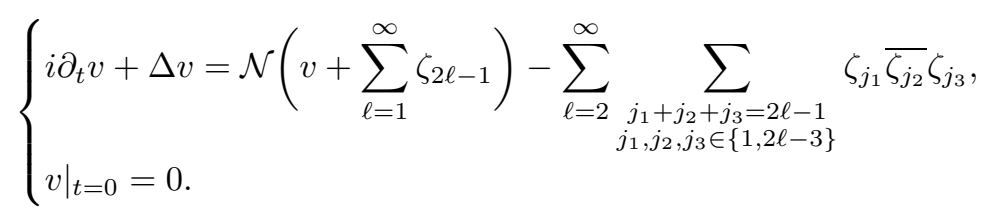

In particular, we would need to worry about the convergence issue of infinite series, and hence there seems to be no simplification in considering the infinite order expansion (1.40).

Another strategy would be to treat $\zeta_{\infty}:=\sum_{\ell=1}^{\infty} \zeta_{2 \ell-1}$ as one stochastic object and write

$$
u=\zeta_{\infty}+v
$$

It follows from (1.28) that $\zeta_{\infty}$ satisfies the following equation:

$$
\left\{\begin{array}{l}
i \partial_{t} \zeta_{\infty}+\Delta \zeta_{\infty}=\sum_{\ell=2}^{\infty} \sum_{\substack{j_{1}+j_{2}+j_{3}=2 \ell-1 \\
j_{1}, j_{2}, j_{3} \in\{1,2 \ell-3\}}} \zeta_{j_{1}} \overline{\zeta_{j_{2}}} \zeta_{j_{3}} \\
\left.\zeta_{\infty}\right|_{t=0}=\phi^{\omega}
\end{array}\right.
$$

Noting that

$$
\sum_{\substack{j_{1}+j_{2}+j_{3}=2 \ell-1 \\ j_{1}, j_{2}, j_{3} \in\{1,2 \ell-3\}}} \zeta_{j_{1}} \overline{\zeta_{j_{2}}} \zeta_{j_{3}}= \begin{cases}\left|z_{1}\right|^{2} z_{1} & \text { when } \ell=2, \\ 2\left|z_{1}\right|^{2} \zeta_{2 \ell-3}+z_{1}^{2} \overline{\zeta_{2 \ell-3}} & \text { when } \ell \geq 3\end{cases}
$$

we can rewrite (1.41) as

$$
\left\{\begin{array}{l}
i \partial_{t} \zeta_{\infty}+\Delta \zeta_{\infty}=2\left|z_{1}\right|^{2} \zeta_{\infty}+z_{1}^{2} \overline{\zeta_{\infty}}-2\left|z_{1}\right|^{2} z_{1} \\
\left.\zeta_{\infty}\right|_{t=0}=\phi^{\omega}
\end{array}\right.
$$

and thus $v:=u-\zeta_{\infty}$ satisfies

$$
\left\{\begin{array}{l}
i \partial_{t} v+\Delta v=\mathcal{N}\left(v+\zeta_{\infty}\right)-2\left|z_{1}\right|^{2} \zeta_{\infty}-z_{1}^{2} \overline{\zeta_{\infty}}+2\left|z_{1}\right|^{2} z_{1}, \\
\left.v\right|_{t=0}=0
\end{array}\right.
$$

The equations (1.42) and (1.43) do not particularly appear to be in such a friendly format. Namely, studying (1.42) and (1.43) does not seem to provide a simplification over the case-by-case analysis (1.35) for the equation (1.34) for each fixed $k \in \mathbb{N}$.

In a recent work [40, the second author (with Tzvetkov and Y. Wang) proved invariance of the white noise for the (renormalized) cubic fourth order NLS on the circle. One novelty of this work is that we introduced an infinite sequence $\left\{z_{2 \ell-1}^{\text {res }}\right\}_{\ell \in \mathbb{N}}$ of stochastic $(2 \ell-1)$-linear objects (depending only on the random initial data) and considered the following expansion of an infinite order:

$$
u=\sum_{\ell=1}^{\infty} z_{2 \ell-1}^{\mathrm{res}}+v
$$


For this problem, it turned out that $z_{\infty}^{\text {res }}:=\sum_{\ell=1}^{\infty} z_{2 \ell-1}^{\text {res }}$ satisfies a particularly simple equation 20 Then by treating $z_{\infty}^{\text {res }}$ as one stochastic object, we wrote a solution $u$ as $u=z_{\infty}^{\text {res }}+v$, which led to the factorization

$$
\phi^{\omega} \in H^{s}(\mathbb{T}) \longmapsto z_{\infty}^{\mathrm{res}}=\sum_{\ell=1}^{\infty} z_{2 \ell-1}^{\mathrm{res}} \longmapsto v \in C\left(\mathbb{R} ; L^{2}(\mathbb{T})\right)
$$

for $s<-\frac{1}{2}$, where $\phi^{\omega}$ denotes the Gaussian white noise on the circle.

Remark 1.12. In 44, the third author (with Y. Wang) recently studied probabilistic local well-posedness of NLS on $\mathbb{R}^{d}$ within the framework of the $L^{p}$-based Sobolev spaces, using the dispersive estimate. In the context of the cubic NLS (1.1) on $\mathbb{R}^{3}$, their result yields almost sure local existence of a unique solution $u$ for the randomized initial data $\phi^{\omega} \in H^{s}\left(\mathbb{R}^{3}\right)$, provided $s \geq 0$. In particular, the argument in 44] allows us to consider random initial data of lower regularities than Theorems A, 1.3, and 1.8. Note that, in 44, it was shown that the solution $u$ only belongs to $C\left([0, T] ; W^{s, 4}\left(\mathbb{R}^{3}\right)\right)$, almost surely. We point out that a slight adaptation of the work [39] by the second and third authors (with Y. Wang) shows that the solution $u$ indeed lies in $C\left([0, T] ; H^{s}\left(\mathbb{R}^{3}\right)\right)$.

As compared to [4], the argument presented in this paper provides extra regularity information, namely the decomposition (1.33) of the solution $u$ with the terms of increasing regularities: $\zeta_{2 \ell-1} \in X_{\mathrm{loc}}^{\alpha_{\ell} \cdot s-}$ and $v \in X^{\frac{1}{2}}([0, T])$. In particular, the residual term $v$ lies in the (sub)critical regularity leaving us a possibility of adapting deterministic techniques to study its further properties.

This paper is organized as follows. In Section 2, we recall probabilistic and deterministic lemmas along with the definitions of the basic function spaces. In Section 3, we study the regularity properties of the second order term $z_{3}$ in (1.10). In Section 4, we further investigate the regularity properties of the higher order terms $z_{5}$ and $z_{7}$ and the unbalanced higher order terms $\zeta_{2 k-1}$. Note that the analysis on $z_{5}$ and $z_{7}$ contains part22 of the case-by-case analysis (1.15) needed for proving Theorem 1.3. In Section [5] we then carry out the rest of the case-by-case analysis (1.15) and prove Theorem 1.3. In Section 6, we briefly describe the proof of Theorem 1.8 by indicating how the analysis in the previous sections can lead to the proof. In Appendix A we prove the deterministic non-smoothing of the Duhamel integral operator discussed in Remark 1.5.

Notation. We use $a+($ and $a-$ ) to denote $a+\varepsilon$ (and $a-\varepsilon$, respectively) for arbitrarily small $\varepsilon \ll 1$, where an implicit constant is allowed to depend on $\varepsilon>0$ (and it usually diverges as $\varepsilon \rightarrow 0$ ).

Given a Banach space $B$ of temporal functions, we use the following shorthand notation: $B_{T}:=B([0, T])$. For example, $L_{T}^{q} L_{x}^{r}=L_{t}^{q}\left([0, T] ; L_{x}^{r}\left(\mathbb{R}^{3}\right)\right)$.

\footnotetext{
${ }^{20}$ In fact, the series $z_{\infty}^{\text {res }}=\sum_{\ell=1}^{\infty} z_{2 \ell-1}^{\text {res }}$ corresponds to the power series expansion of the resonant cubic fourth order NLS. We point out that $z_{\infty}^{\text {res }}$ does not belong to the span of Wiener homogeneous chaoses of any finite order.

${ }^{21}$ By slightly tweaking the argument, we can easily place $v$ in $X^{\frac{1}{2}+}([0, T])$.

${ }^{22}$ Note that $z_{7}$ defined in (1.22) contains the contribution from $z_{j_{1}} \overline{z_{j_{2}}} z_{j_{3}}$ with $\left(j_{1}, j_{2}, j_{3}\right)=$ $(1,3,3)$ up to permutations.
} 
Let $\eta: \mathbb{R} \rightarrow[0,1]$ be an even, smooth cutoff function supported on $\left[-\frac{8}{5}, \frac{8}{5}\right]$ such that $\eta \equiv 1$ on $\left[-\frac{5}{4}, \frac{5}{4}\right]$. Given a dyadic number $N \geq 1$, we set $\eta_{1}(\xi)=\eta(|\xi|)$ and

$$
\eta_{N}(\xi)=\eta\left(\frac{|\xi|}{N}\right)-\eta\left(\frac{2|\xi|}{N}\right)
$$

for $N \geq 2$. Then, we define the Littlewood-Paley projection operator $\mathbf{P}_{N}$ as the Fourier multiplier operator with symbol $\eta_{N}$. In the following, we use the convention that capital letters denote dyadic numbers. For example, $N=2^{n}$ for some $n \in$ $\mathbb{N}_{0}:=\mathbb{N} \cup\{0\}$.

Given dyadic numbers $N_{1}, \ldots, N_{4} \in 2^{\mathbb{N}_{0}}$, we set $N_{\max }:=\max _{j=1, \ldots, 4} N_{j}$. We also use the shorthand notation $f_{N}=\mathbf{P}_{N} f$. For example, we have $z_{1, N_{j}}=\mathbf{P}_{N_{j}} z_{1}$.

\section{StRichartz ESTIMATES AND FUNCTION SPACES}

2.1. Probabilistic Strichartz estimates. First, we recall the usual Strichartz estimates on $\mathbb{R}^{3}$ for the reader's convenience. We say that a pair $(q, r)$ is Schrödinger admissible if it satisfies

$$
\frac{2}{q}+\frac{3}{r}=\frac{3}{2}
$$

with $2 \leq q, r \leq \infty$. Then, the following Strichartz estimates are known to hold [20, 28, 46, 49]:

$$
\|S(t) \phi\|_{L_{t}^{q} L_{x}^{r}\left(\mathbb{R} \times \mathbb{R}^{3}\right)} \lesssim\|\phi\|_{L_{x}^{2}\left(\mathbb{R}^{3}\right)} .
$$

It follows from (2.1) and Sobolev's inequality that

$$
\|S(t) \phi\|_{L_{t, x}^{p}\left(\mathbb{R} \times \mathbb{R}^{3}\right)} \lesssim\left\||\nabla|^{\frac{3}{2}-\frac{5}{p}} \phi\right\|_{L_{x}^{2}\left(\mathbb{R}^{3}\right)}
$$

for $p \geq \frac{10}{3}$. We will use the following admissible pairs in this paper:

$$
(\infty, 2),\left(5, \frac{30}{11}\right),\left(\frac{10}{3} \frac{10}{3}\right),(2+, 6-) .
$$

In particular, by Sobolev's inequality, we have

$$
W^{\frac{1}{2}, \frac{30}{11}}\left(\mathbb{R}^{3}\right) \hookrightarrow L^{5}\left(\mathbb{R}^{3}\right) .
$$

One of the important key ingredients for probabilistic well-posedness is the probabilistic Strichartz estimates. Such probabilistic estimates were first exploited by McKean [34] and Bourgain [5]. In the following, we state the probabilistic Strichartz estimates under the Wiener randomization (1.3). See [1] for the proofs.

Lemma 2.1. Given $\phi$ on $\mathbb{R}^{3}$, let $\phi^{\omega}$ be its Wiener randomization defined in (1.3). Then, given finite $q \geq 2$ and $2 \leq r \leq \infty$, there exist $C, c>0$ such that

$$
P\left(\left\|S(t) \phi^{\omega}\right\|_{L_{t}^{q} L_{x}^{r}\left([0, T] \times \mathbb{R}^{3}\right)}>\lambda\right) \leq C \exp \left(-c \frac{\lambda^{2}}{T^{\frac{2}{q}\|\phi\|_{H^{s}}^{2}}}\right)
$$

for all $T>0$ and $\lambda>0$ with (i) $s=0$ if $r<\infty$ and (ii) $s>0$ if $r=\infty$.

A similar estimate holds when $q=\infty$ (with $s>0$ ), but we will not need it in this paper. See [38. We also need the following lemma on the control of the size of $H^{s}$-norm of $\phi^{\omega}$. 
Lemma 2.2. Given $\phi \in H^{s}\left(\mathbb{R}^{3}\right)$, let $\phi^{\omega}$ be its Wiener randomization defined in (1.3). Then, we have

$$
P\left(\left\|\phi^{\omega}\right\|_{H^{s}\left(\mathbb{R}^{3}\right)}>\lambda\right) \leq C \exp \left(-c \frac{\lambda^{2}}{\|\phi\|_{H^{s}}^{2}}\right)
$$

for all $\lambda>0$.

2.2. Function spaces and their properties. In this subsection, we go over the basic definitions and properties of the function spaces used for the Fourier restriction norm method (i.e., analysis involving the $X^{s, b}$-spaces introduced in [4]) adapted to the space of functions of bounded $p$-variation and its pre-dual, introduced and developed by Tataru, Koch, and their collaborators [22,24, 31. We refer readers to 22,24 for the proofs of the basic properties. See also [2].

Let $\mathcal{Z}$ be the set of finite partitions $-\infty<t_{0}<t_{1}<\cdots<t_{K} \leq \infty$ of the real line. By convention, we set $u\left(t_{K}\right):=0$ if $t_{K}=\infty$. We use $\mathbf{1}_{I}$ to denote the sharp characteristic function of a set $I \subset \mathbb{R}$.

Definition 2.3. Let $1 \leq p<\infty$.

(i) We define a $U^{p}$-atom to be a step function $a: \mathbb{R} \rightarrow L^{2}\left(\mathbb{R}^{3}\right)$ of the form

$$
a=\sum_{k=1}^{K} \phi_{k-1} \mathbf{1}_{\left[t_{k-1}, t_{k}\right)},
$$

where $\left\{t_{k}\right\}_{k=0}^{K} \in \mathcal{Z}$ and $\left\{\phi_{k}\right\}_{k=0}^{K-1} \subset L^{2}\left(\mathbb{R}^{3}\right)$ with $\sum_{k=0}^{K-1}\left\|\phi_{k}\right\|_{L^{2}}^{p}=1$. Furthermore, we define the atomic space $U^{p}=U^{p}\left(\mathbb{R} ; L^{2}\left(\mathbb{R}^{3}\right)\right)$ by

$$
U^{p}:=\left\{u: \mathbb{R} \rightarrow L^{2}\left(\mathbb{R}^{3}\right): u=\sum_{j=1}^{\infty} \lambda_{j} a_{j} \text { for } U^{p} \text {-atoms } a_{j},\left\{\lambda_{j}\right\}_{j \in \mathbb{N}} \in \ell^{1}(\mathbb{N} ; \mathbb{C})\right\}
$$

with the norm

$$
\|u\|_{U^{p}}:=\inf \left\{\sum_{j=1}^{\infty}\left|\lambda_{j}\right|: u=\sum_{j=1}^{\infty} \lambda_{j} a_{j} \text { for } U^{p} \text {-atoms } a_{j},\left\{\lambda_{j}\right\}_{j \in \mathbb{N}} \in \ell^{1}(\mathbb{N} ; \mathbb{C})\right\},
$$

where the infimum is taken over all possible representations for $u$.

(ii) We define $V^{p}=V^{p}\left(\mathbb{R} ; L^{2}\left(\mathbb{R}^{3}\right)\right)$ to be the space of functions $u: \mathbb{R} \rightarrow L^{2}\left(\mathbb{R}^{3}\right)$ of bounded $p$-variation with the standard $p$-variation norm:

$$
\|u\|_{V^{p}}:=\sup _{\left\{t_{k}\right\}_{k=0}^{K} \in \mathcal{Z}}\left(\sum_{k=1}^{K}\left\|u\left(t_{k}\right)-u\left(t_{k-1}\right)\right\|_{L^{2}}^{p}\right)^{\frac{1}{p}} .
$$

By convention, we impose that the limits $\lim _{t \rightarrow \pm \infty} u(t)$ exist in $L^{2}\left(\mathbb{R}^{3}\right)$.

(iii) Let $V_{\mathrm{rc}}^{p}$ be the closed subspace of $V^{p}$ of all right-continuous functions $u \in V^{p}$ with $\lim _{t \rightarrow-\infty} u(t)=0$.

(iv) We define $U_{\Delta}^{p}:=S(t) U^{p}$ (and $V_{\Delta}^{p}:=S(t) V^{p}$, respectively) to be the space of all functions $u: \mathbb{R} \rightarrow L^{2}\left(\mathbb{R}^{3}\right)$ such that $t \rightarrow S(-t) u(t)$ is in $U^{p}$ (and in $V^{p}$, respectively) with the norms

$$
\|u\|_{U_{\Delta}^{p}}:=\|S(-t) u\|_{U^{p}} \quad \text { and } \quad\|u\|_{V_{\Delta}^{p}}:=\|S(-t) u\|_{V^{p}} .
$$

The closed subspace $V_{\mathrm{rc}, \Delta}^{p}$ is defined in an analogous manner. 
Recall the following inclusion relation: for $1 \leq p<q<\infty$,

$$
U^{p} \hookrightarrow V_{\mathrm{rc}}^{p} \hookrightarrow U^{q} \hookrightarrow L^{\infty}\left(\mathbb{R} ; L^{2}\left(\mathbb{R}^{3}\right)\right) .
$$

The space $V^{p}$ is the classical space of functions of bounded $p$-variation, and the space $U^{p}$ appears as the pre-dual of $V^{p^{\prime}}$ with $\frac{1}{p}+\frac{1}{p^{\prime}}=1,1<p<\infty$. Their duality relation and the atomic structure of the $U^{p}$-space turned out to be very effective in studying dispersive PDEs in critical settings.

We are now ready to define the solution spaces.

\section{Definition 2.4.}

(i) Let $s \in \mathbb{R}$. We define $X^{s}(\mathbb{R})$ to be the closure of $C\left(\mathbb{R} ; H^{s}\left(\mathbb{R}^{3}\right)\right) \cap U_{\Delta}^{2}$ with respect to the $X^{s}$-norm defined by

$$
\|u\|_{X^{s}(\mathbb{R})}:=\left(\sum_{\substack{N \geq 1 \\ \text { dyadic }}} N^{2 s}\left\|\mathbf{P}_{N} u\right\|_{U_{\Delta}^{2} L^{2}}^{2}\right)^{\frac{1}{2}} .
$$

(ii) Let $s \in \mathbb{R}$. We define $Y^{s}(\mathbb{R})$ to be the space of all functions $u \in C\left(\mathbb{R} ; H^{s}\left(\mathbb{R}^{3}\right)\right)$ such that the map $t \mapsto \mathbf{P}_{N} u$ lies in $V_{\mathrm{rc}, \Delta}^{2} H^{s}$ for any $N \in 2^{\mathbb{N}_{0}}$ and $\|u\|_{Y^{s}(\mathbb{R})}<\infty$, where the $Y^{s}$-norm is defined by

$$
\|u\|_{Y^{s}(\mathbb{R})}:=\left(\sum_{\substack{N \geq 1 \\ \text { dyadic }}} N^{2 s}\left\|\mathbf{P}_{N} u\right\|_{V_{\Delta}^{2} L^{2}}^{2}\right)^{\frac{1}{2}} .
$$

Recall the following embeddings:

$$
U_{\Delta}^{2} H^{s} \hookrightarrow X^{s} \hookrightarrow Y^{s} \hookrightarrow V_{\Delta}^{2} H^{s} \hookrightarrow U_{\Delta}^{p} H^{s}
$$

for $p>2$.

Given an interval $I \subset \mathbb{R}$, we define the local-in-time versions $X^{s}(I)$ and $Y^{s}(I)$ of these spaces as restriction norms. For example, we define the $X^{s}(I)$-norm by

$$
\|u\|_{X^{s}(I)}=\inf \left\{\|v\|_{X^{s}(\mathbb{R})}:\left.v\right|_{I}=u\right\} .
$$

We also define the norm for the non-homogeneous term on an interval $I=\left[t_{0}, t_{1}\right)$ :

$$
\|F\|_{N^{s}(I)}=\left\|\int_{t_{0}}^{t} S\left(t-t^{\prime}\right) F\left(t^{\prime}\right) d t^{\prime}\right\|_{X^{s}(I)} .
$$

We conclude this section by presenting some basic estimates involving these function spaces. See [2,22,24] for the proofs.

Lemma 2.5. Let $s \in \mathbb{R}$ and $T \in(0, \infty]$. Then, the following linear estimates hold:

$$
\begin{aligned}
\|S(t) \phi\|_{X^{s}([0, T])} & \leq\|\phi\|_{H^{s}}, \\
\|F\|_{N^{s}([0, T])} & \leq \sup _{\substack{w \in Y^{-s}([0, T]) \\
\|w\|_{Y^{-s}([0, T])}}}\left|\int_{0}^{T}\langle F(t), w(t)\rangle_{L_{x}^{2}} d t\right|
\end{aligned}
$$

for any $\phi \in H^{s}\left(\mathbb{R}^{3}\right)$ and $F \in L^{1}\left([0, T] ; H^{s}\left(\mathbb{R}^{3}\right)\right)$.

The transference principle [22, Proposition 2.19] and the interpolation lemma [22, Proposition 2.20] applied on the Strichartz estimates (2.1) and (2.2) imply the following estimates. 
Lemma 2.6. Given any admissible pair $(q, r)$ with $q>2$ and $p \geq \frac{10}{3}$, we have

$$
\begin{aligned}
\|u\|_{L_{t}^{q} L_{x}^{r}} & \lesssim\|u\|_{Y^{0}}, \\
\|u\|_{L_{t, x}^{p}} & \lesssim\left\|\left.\nabla\right|^{\frac{3}{2}-\frac{5}{p}} u\right\|_{Y^{0}} .
\end{aligned}
$$

Similarly, the bilinear refinement of the Strichartz estimate [6, 14, 41] implies the following bilinear estimate.

Lemma 2.7. Let $N_{1}, N_{2} \in 2^{\mathbb{N}_{0}}$ with $N_{1} \leq N_{2}$. Then, we have

$$
\left\|\mathbf{P}_{N_{1}} u_{1} \mathbf{P}_{N_{2}} u_{2}\right\|_{L_{t}^{2}\left([0, T] ; L_{x}^{2}\right)} \lesssim T^{0+} N_{1}^{1-} N_{2}^{-\frac{1}{2}+}\left\|\mathbf{P}_{N_{1}} u_{1}\right\|_{Y^{0}([0, T])}\left\|\mathbf{P}_{N_{2}} u_{2}\right\|_{Y^{0}([0, T])}
$$

for any $T>0$ and $u_{1}, u_{2} \in Y^{0}([0, T])$.

Proof. From the bilinear refinement of the Strichartz estimate [6, 14 and the transference principle, we have

$$
\left\|\mathbf{P}_{N_{1}} u_{1} \mathbf{P}_{N_{2}} u_{2}\right\|_{L_{t, x}^{2}} \lesssim N_{1}^{1-} N_{2}^{-\frac{1}{2}+}\left\|\mathbf{P}_{N_{1}} u_{1}\right\|_{Y^{0}}\left\|\mathbf{P}_{N_{2}} u_{2}\right\|_{Y^{0}}
$$

for all $u_{1}, u_{2} \in Y^{0}$. See 2 for the proof of (2.5). On the other hand, it follows from Hölder's and Sobolev's inequalities that

$$
\begin{aligned}
\left\|\mathbf{P}_{N_{1}} u_{1} \mathbf{P}_{N_{2}} u_{2}\right\|_{L_{T}^{2} L_{x}^{2}} & \lesssim T^{\frac{1}{2}}\left\|\mathbf{P}_{N_{1}} u_{1}\right\|_{L_{T}^{\infty} L_{x}^{4}}\left\|\mathbf{P}_{N_{2}} u_{2}\right\|_{L_{T}^{\infty} L_{x}^{4}} \\
& \lesssim T^{\frac{1}{2}} N_{1}^{\frac{3}{4}} N_{2}^{\frac{3}{4}}\left\|\mathbf{P}_{N_{1}} u_{1}\right\|_{Y_{T}^{0}}\left\|\mathbf{P}_{N_{2}} u_{2}\right\|_{Y_{T}^{0}} .
\end{aligned}
$$

Then, the estimate (2.4) follows from interpolating (2.5) and (2.6).

\section{ON THE SECOND ORDER TERM $z_{3}$}

In this and the next sections, we study the regularity properties of the various stochastic terms that appear in the iterative procedures. Given $\phi \in H^{s}\left(\mathbb{R}^{3}\right)$, let $\phi^{\omega}$ be the Wiener randomization of $\phi$ defined in (1.3) and set

$$
z_{1}=S(t) \phi^{\omega} .
$$

In this section, we study the regularity properties of the second order term:

$$
z_{3}=-i \int_{0}^{t} S\left(t-t^{\prime}\right)\left|z_{1}\right|^{2} z_{1}\left(t^{\prime}\right) d t^{\prime}
$$

We first present the proof of Proposition 1.2. We follow closely the argument in [2]. Proof of Proposition 1.2. (i) By Lemma 2.5, the estimate (1.11) follows once we prove

$$
\left|\int_{0}^{T} \int_{\mathbb{R}^{3}}\langle\nabla\rangle^{\sigma}\left(z_{1} \overline{z_{1}} z_{1}\right) \bar{w} d x d t\right| \leq T^{\theta} C\left(\omega,\|\phi\|_{H^{s}}\right)
$$

for some almost surely finite constant $C\left(\omega,\|\phi\|_{H^{s}}\right)>0$ and $\theta>0$, where $\|w\|_{Y_{T}^{0}} \leq 1$. In the following, we drop the complex conjugate when it does not play any role.

Define $A_{3}^{s}(T)$ by

$$
\left\|z_{1}\right\|_{A_{3}^{s}(T)}:=\max \left(\left\|\langle\nabla\rangle^{s} z_{1}\right\|_{L_{T}^{\frac{30}{7}}+{ }_{L_{x}^{7}}^{\frac{30}{7}}},\left\|\langle\nabla\rangle^{s} z_{1}\right\|_{L_{T, x}^{4}},\left\|z_{1}(0)\right\|_{H^{s}}\right) .
$$


Then, by applying the dyadic decomposition, it suffices to prove that

$$
\left|\int_{0}^{T} \int_{\mathbb{R}^{3}} \mathbf{P}_{N_{1}} z_{1} \cdot \mathbf{P}_{N_{2}} z_{1} \cdot N_{3}^{\sigma} \mathbf{P}_{N_{3}} z_{1} \cdot \mathbf{P}_{N_{4}} w d x d t\right| \leq T^{\theta} N_{\max }^{0-} C\left(\left\|z_{1}\right\|_{A_{3}^{s}(T)}\right)
$$

for all $N_{1}, \ldots, N_{4} \in 2^{\mathbb{N}_{0}}$ with $N_{3} \geq N_{2} \geq N_{1}$. Once we prove (3.4), the desired estimate (3.2) follows from summing (3.4) over dyadic blocks and applying Lemmas 2.1 and 2.2. Recall our shorthand notation: $z_{1, N_{j}}=\mathbf{P}_{N_{j}} z_{1}$ and $w_{N_{4}}=\mathbf{P}_{N_{4}} w$.

In the following, we assume that

$$
\sigma<2 s \quad \text { and } \quad 0 \leq s<1 .
$$

Case $1\left(N_{2} \sim N_{3}\right)$. By Hölder's inequality and Lemma 2.6, we have

$$
\begin{aligned}
\operatorname{LHS} \text { of }(3.4) & \lesssim\left\|z_{1, N_{1}}\right\|_{L_{T, x}^{\frac{30}{7}}}\left(\prod_{j=2}^{3}\left\|\langle\nabla\rangle^{\frac{\sigma}{2}} z_{1, N_{j}}\right\|_{L_{T, x}^{\frac{30}{7}}}\right)\left\|w_{N_{4}}\right\|_{L_{T, x}^{\frac{10}{3}}} \\
& \leq T^{0+} N_{\max }^{0-} C\left(\left\|z_{1}\right\|_{A_{3}^{s}(T)}\right)\left\|w_{N_{4}}\right\|_{Y_{T}^{0}},
\end{aligned}
$$

provided that (3.5) holds.

Case $2\left(N_{3} \sim N_{4} \gg N_{1}, N_{2}\right)$.

Subcase 2.a $\left(N_{1}, N_{2} \ll N_{3}^{\frac{1}{2}}\right)$. By Cauchy-Schwarz' inequality and Lemma 2.7 followed by Lemma $2.5 \sqrt{23}$ we have

$$
\begin{aligned}
\text { LHS of (3.4) } & \leq N_{3}^{\sigma}\left\|z_{1, N_{1}} z_{1, N_{3}}\right\|_{L_{T, x}^{2}}\left\|z_{1, N_{2}} w_{N_{4}}\right\|_{L_{T, x}^{2}} \\
& \lesssim T^{0+} N_{1}^{1-s-} N_{2}^{1-s-} N_{3}^{\sigma-s-1+}\left(\prod_{j=1}^{3}\left\|\mathbf{P}_{N_{j}} \phi^{\omega}\right\|_{H^{s}}\right)\left\|w_{N_{4}}\right\|_{Y_{T}^{0}} \\
& \lesssim T^{0+} N_{3}^{\sigma-2 s+} \prod_{j=1}^{3}\left\|\mathbf{P}_{N_{j}} \phi^{\omega}\right\|_{H^{s}} \\
& \leq T^{0+} N_{\max }^{0-} C\left(\left\|z_{1}\right\|_{A_{3}^{s}(T)}\right),
\end{aligned}
$$

provided that (3.5) holds.

Subcase 2.b $\left(N_{1}, N_{2} \gtrsim N_{3}^{\frac{1}{2}}\right)$. By Hölder's inequality and Lemma 2.6, we have

$$
\begin{aligned}
\text { LHS of }(\underline{3.4)} & \leq N_{3}^{\sigma}\left(\prod_{j=1}^{3}\left\|z_{1, N_{j}}\right\|_{L_{T, x}^{\frac{30}{7}}}\right)\left\|w_{N_{4}}\right\|_{L_{T, x}^{\frac{10}{3}}} \\
& \leq T^{0+} N_{1}^{-s} N_{2}^{-s} N_{3}^{\sigma-s} C\left(\left\|z_{1}\right\|_{A_{3}^{s}(T)}\right) \\
& \leq T^{0+} N_{\max }^{0-} C\left(\left\|z_{1}\right\|_{A_{3}^{s}(T)}\right),
\end{aligned}
$$

provided that (3.5) holds.

\footnotetext{
${ }^{23}$ In the remaining part of this paper, we repeatedly apply this argument when there is a frequency separation. We shall simply refer to it as the "bilinear Strichartz estimate" argument.
} 
Subcase 2.c $\left(N_{2} \gtrsim N_{3}^{\frac{1}{2}} \gg N_{1}\right)$. By the bilinear Strichartz estimate, we have

$$
\begin{aligned}
\text { LHS of (13.4) } & \leq N_{3}^{\sigma}\left\|z_{1, N_{1}} w_{N_{4}}\right\|_{L_{T, x}^{2}} \prod_{j=2}^{3}\left\|z_{1, N_{j}}\right\|_{L_{T, x}^{4}} \\
& \lesssim T^{0+} N_{1}^{1-s-} N_{2}^{-s} N_{3}^{\sigma-s-\frac{1}{2}+}\left\|\mathbf{P}_{N_{1}} \phi^{\omega}\right\|_{H^{s}}\left(\prod_{j=2}^{3}\left\|\langle\nabla\rangle^{s} z_{1, N_{j}}\right\|_{L_{T, x}^{4}}\right)\left\|w_{N_{4}}\right\|_{Y_{T}^{0}} \\
& \leq T^{0+} N_{3}^{\sigma-2 s+} C\left(\left\|z_{1}\right\|_{A_{3}^{s}(T)}\right) \\
& \leq T^{0+} N_{\max }^{0-} C\left(\left\|z_{1}\right\|_{A_{3}^{s}(T)}\right),
\end{aligned}
$$

provided that (3.5) holds.

Therefore, putting all the cases together, we obtain (3.4).

(ii) Given $N \gg 1$ and small $\ell>0$, consider the following deterministic initial condition $\phi$ whose Fourier transform is given by

$$
\widehat{\phi}(\xi)=\mathbf{1}_{N e_{1}+\ell Q}(\xi)+\mathbf{1}_{(N+100 \ell) e_{1}+\ell Q}(\xi)+\mathbf{1}_{N e_{1}+100 \ell e_{2}+\ell Q}(\xi),
$$

where $Q=\left(-\frac{1}{2}, \frac{1}{2}\right]^{3}, e_{1}=(1,0,0)$, and $e_{2}=(0,1,0)$. By taking $\ell>0$ sufficiently small, we have supp $\widehat{\phi} \subset N e_{1}+Q$, and thus we can neglect the effect of the randomization in (1.3) since all three terms on the right-hand side will be multiplied by a common random number $g_{N e_{1}}$. Without loss of generality, we assume that $g_{N e_{1}}=1$ in the following.

We estimate from below the contribution to $\mathbf{1}_{Q_{N, \ell}}(\xi) \widehat{z}_{3}(t, \xi)$, where

$$
Q_{N, \ell}=N e_{1}+100 \ell\left(e_{1}+e_{2}\right)+\ell Q .
$$

From (3.1), we have

$$
\mathbf{1}_{Q_{N, \ell}}(\xi) \widehat{z}_{3}(t, \xi)
$$

$$
=-i \mathbf{1}_{Q_{N, \ell}}(\xi) e^{-i t|\xi|^{2}} \int_{0}^{t} \int_{\xi=\xi_{1}-\xi_{2}+\xi_{3}} e^{i t^{\prime} \Phi(\bar{\xi})} \widehat{\phi^{\omega}}\left(\xi_{1}\right) \widehat{\widehat{\phi}^{\omega}}\left(\xi_{2}\right) \widehat{\phi^{\omega}}\left(\xi_{3}\right) d \xi_{1} d \xi_{2} d t^{\prime},
$$

where the phase function $\Phi(\bar{\xi})$ is given by

$$
\Phi(\bar{\xi})=\Phi\left(\xi, \xi_{1}, \xi_{2}, \xi_{3}\right)=|\xi|^{2}-\left|\xi_{1}\right|^{2}+\left|\xi_{2}\right|^{2}-\left|\xi_{3}\right|^{2}=2\left\langle\xi-\xi_{1}, \xi-\xi_{3}\right\rangle_{\mathbb{R}^{3}} .
$$

Then, it follows that the only non-trivial contribution to (3.6) appears if

$$
\xi_{1} \in(N+100 \ell) e_{1}+\ell Q, \quad \xi_{2} \in N e_{1}+\ell Q, \quad \xi_{3} \in N e_{1}+100 \ell e_{2}+\ell Q
$$

(up to the permutation $\xi_{1} \leftrightarrow \xi_{3}$ ). In this case, we have $|\Phi(\bar{\xi})| \ll 1$ and thus

$$
\operatorname{Re} e^{i t^{\prime} \Phi(\bar{\xi})} \geq \frac{1}{2}
$$

for all $t^{\prime} \in[0,1]$.

Now, recall the following lemma on the convolution.

Lemma 3.1. There exists $c>0$ such that

$$
\mathbf{1}_{a+\ell Q} * \mathbf{1}_{b+\ell Q}(\xi) \geq c \ell^{3} \mathbf{1}_{a+b+\ell Q}(\xi)
$$

for all $a, b, \xi \in \mathbb{R}^{3}$. 
By applying Lemma 3.1 to (3.6) with (3.8) and (3.9), we obtain

$$
\left|\mathbf{1}_{Q_{N, \ell}}(\xi) \widehat{z}_{3}(t, \xi)\right| \gtrsim t \ell^{6} \mathbf{1}_{Q_{N, \ell}}(\xi) .
$$

Therefore, for any $\sigma>0$, we have

$$
\left\|z_{3}\right\|_{X^{\sigma}([0,1])} \gtrsim\left\|z_{3}\right\|_{L_{t}^{\infty}\left([0,1] ; H^{\sigma}\right)} \gtrsim \ell^{\frac{15}{2}} N^{\sigma} \longrightarrow \infty
$$

as $N \rightarrow \infty$, while $\|\phi\|_{L^{2}} \sim \ell^{\frac{3}{2}}$ remains bounded. This in particular implies that when $s=0$, the estimate (1.11) cannot hold for any $\sigma>0$. This proves part (ii).

Remark 3.2. It follows from the proof of Proposition 1.2(i) that

$$
\left\|\mathcal{I}\left(u_{1}, u_{2}, u_{3}\right)\right\|_{X^{\sigma}([0,1])} \lesssim \prod_{j=1}^{3}\left\|u_{j}\right\|_{A_{3}^{s}(T)},
$$

where $\mathcal{I}\left(u_{1}, u_{2}, u_{3}\right)$ is as in (1.17). In particular, the left-hand side of (1.18) is finite for $u_{1}, u_{2}, u_{3} \in \mathcal{R}^{s}$. The only probabilistic component in the proof of Proposition 1.2 (i) appears in applying Lemmas 2.1 and 2.2 to control the $A_{3}^{s}(T)$-norm of $z_{1}$ in terms of the $H^{s}$-norm of $\phi$. In this sense, we exploit the randomization only at the linear level.

On the one hand, Proposition 1.2 shows that $z_{3}$ controls almost $2 s$ derivatives. On the other hand, we need to measure $z_{3}$ in the $X^{2 s-}$-norm, which controls only the admissible space-time Lebesgue norms (with $2 s-$ derivatives) via Lemma 2.6. The following lemma breaks this rigidity by giving up a control on derivatives. In particular, it allows us to control a wider range of space-time Lebesgue norms of $z_{3}$. The main idea is to use the dispersive estimate for the linear Schrödinger operator:

$$
\|S(t) f\|_{L_{x}^{r}} \lesssim|t|^{-\frac{3}{2}\left(1-\frac{2}{r}\right)}\|f\|_{L_{x}^{r^{\prime}}}
$$

This allows us to reduce the analysis to a product of the random linear solution $z_{1}=S(t) \phi^{\omega}$ and apply Lemma 2.1.

Lemma 3.3. Let $s \geq 0$. Given $\phi \in H^{s}\left(\mathbb{R}^{3}\right)$, let $\phi^{\omega}$ be its Wiener randomization defined in (1.3). Then, for any finite $q, r \geq 1$, we have

$$
\left\|\mathbf{P}_{N} z_{3}\right\|_{L_{T}^{q} L_{x}^{r}} \lesssim \begin{cases}T^{\frac{3}{r}-\frac{1}{2}}\left\|z_{1}\right\|_{L_{T}^{3 q} L_{x}^{3 r^{\prime}}}^{3} & \text { when } 1 \leq r<6, \\ T^{0+} N^{\frac{1}{2}-\frac{3}{r}+}\left\|z_{1}\right\|_{L_{T}^{3 q} L_{x}^{\frac{18}{5}}+}^{3} & \text { when } r \geq 6\end{cases}
$$

for any $T>0$ and $N \in 2^{\mathbb{N}_{0}}$. Note that the right-hand side of (3.11) is almost surely finite thanks to the probabilistic Strichartz estimate (Lemma 2.1).

Proof. We first consider the case $r<6$. From (3.1) and (3.10), we have

$$
\begin{aligned}
\left\|\mathbf{P}_{N} z_{3}\right\|_{L_{T}^{q} L_{x}^{r}} & \leq\left\|\int_{0}^{t}\right\| \mathbf{P}_{N} S\left(t-t^{\prime}\right)\left|z_{1}\right|^{2} z_{1}\left(t^{\prime}\right)\left\|_{L_{x}^{r}} d t^{\prime}\right\|_{L_{T}^{q}} \\
& \lesssim\left\|\int_{0}^{t} \frac{1}{\left|t-t^{\prime}\right|^{\frac{3}{2}-\frac{3}{r}}}\right\| z_{1}\left(t^{\prime}\right)\left\|_{L_{x}^{3 r^{\prime}}}^{3} d t^{\prime}\right\|_{L_{T}^{q}} \\
& \lesssim T^{\frac{3}{r}-\frac{1}{2}}\left\|z_{1}\right\|_{L_{T}^{3 q} L_{x}^{3 r^{\prime}}}^{3}
\end{aligned}
$$


When $r \geq 6$, we proceed as in (3.12), but we apply Sobolev's inequality before applying (3.10):

$$
\begin{aligned}
\left\|\mathbf{P}_{N} z_{3}\right\|_{L_{T}^{q} L_{x}^{r}} & \lesssim\left\|\int_{0}^{t}\right\| \mathbf{P}_{N} S\left(t-t^{\prime}\right)\left|z_{1}\right|^{2} z_{1}\left(t^{\prime}\right)\left\|_{L_{x}^{r}} d t^{\prime}\right\|_{L_{T}^{q}} \\
& \lesssim N^{\frac{1}{2}-\frac{3}{r}+}\left\|\int_{0}^{t}\right\| \mathbf{P}_{N} S\left(t-t^{\prime}\right)\left|z_{1}\right|^{2} z_{1}\left(t^{\prime}\right)\left\|_{L_{x}^{6-}} d t^{\prime}\right\|_{L_{T}^{q}} \\
& \lesssim N^{\frac{1}{2}-\frac{3}{r}+}\left\|\int_{0}^{t} \frac{1}{\left|t-t^{\prime}\right|^{1-}}\right\| z_{1}\left(t^{\prime}\right)\left\|_{L_{x}^{\frac{18}{5}}+}^{3} d t^{\prime}\right\|_{L_{T}^{q}} \\
& \lesssim T^{0+} N^{\frac{1}{2}-\frac{3}{r}+}\left\|z_{1}\right\|_{L_{T}^{3 q} L_{x}^{\frac{18}{5}+}}^{3}
\end{aligned}
$$

This completes the proof of Lemma 3.3

\section{ON THE HIGHER ORDER TERMS}

In this section, we study the regularity properties of the higher order terms.

4.1. On the third order term $z_{5}$. In this subsection, we study the third order term:

$$
z_{5}=-i \sum_{\substack{j_{1}+j_{2}+j_{3}=5 \\ j_{1}, j_{2}, j_{3} \in\{1,3\}}} \int_{0}^{t} S\left(t-t^{\prime}\right) z_{j_{1}} \overline{z_{j_{2}}} z_{j_{3}}\left(t^{\prime}\right) d t^{\prime} .
$$

The following lemma shows that the third order term $z_{5}$ enjoys a gain of extra $\frac{1}{2} s$ derivative as compared to the second order term $z_{3}$ (Proposition 1.2).

Lemma 4.1. Given $0<s<\frac{1}{2}$, let $\phi^{\omega}$ be the Wiener randomization of $\phi \in H^{s}\left(\mathbb{R}^{3}\right)$ defined in (1.3). Then, for any $\sigma<\frac{5}{2} s$, we have

$$
z_{5} \in X_{\mathrm{loc}}^{\sigma}
$$

almost surely. In particular, there exist an almost surely finite constant $C\left(\omega,\|\phi\|_{H^{s}}\right)$ $>0$ and $\theta>0$ such that

$$
\left\|z_{5}\right\|_{X^{\sigma}([0, T])} \leq T^{\theta} C\left(\omega,\|\phi\|_{H^{s}}\right)
$$

for any $T>0$.

Proof. First, note that the only possible combination for $\left(j_{1}, j_{2}, j_{3}\right)$ in (4.1) is $(1,1,3)$ up to permutations. The complex conjugate does not play any role in the subsequent analysis, and hence we drop the complex conjugate sign and simply study

$$
z_{5} \sim \int_{0}^{t} S\left(t-t^{\prime}\right) z_{1} z_{1} z_{3}\left(t^{\prime}\right) d t^{\prime} .
$$

By Lemma 2.5, it suffices to prove that

$$
\left|\int_{0}^{T} \int_{\mathbb{R}^{3}}\langle\nabla\rangle^{\sigma}\left(z_{1} z_{1} z_{3}\right) w d x d t\right| \leq T^{\theta} C\left(\omega,\|\phi\|_{H^{s}}\right)
$$

for some almost surely finite constant $C\left(\omega,\|\phi\|_{H^{s}}\right)>0$ and $\theta>0$, where $\|w\|_{Y_{T}^{0}} \leq 1$. Define $A_{5}^{s}(T)$ by

$$
\left\|z_{1}\right\|_{A_{5}^{s}(T)}:=\max \left(\left\|\langle\nabla\rangle^{s} z_{1}\right\|_{L_{T}^{5+} L_{x}^{5}},\left\|z_{1}(0)\right\|_{H^{s}},\right) .
$$


Then, by applying the dyadic decomposition, it suffices to prove that

$$
\begin{aligned}
\mid \int_{0}^{T} \int_{\mathbb{R}^{3}} N_{\max }^{\sigma} \mathbf{P}_{N_{1}} z_{1} \cdot \mathbf{P}_{N_{2}} z_{1} \cdot & \mathbf{P}_{N_{3}} z_{3} \cdot \mathbf{P}_{N_{4}} w d x d t \mid \\
& \leq T^{\theta} N_{\max }^{0-} C\left(\left\|z_{1}\right\|_{A_{5}^{s}(T)},\left\|z_{3}\right\|_{X_{T}^{2 s-}}\right)
\end{aligned}
$$

for all $N_{1}, \ldots, N_{4} \in 2^{\mathbb{N}_{0}}$. Once we prove (4.4), the desired estimate (4.3) follows from summing over dyadic blocks and applying Lemmas 2.1 and 2.2 and Proposition 1.2. In the following, we fix

$$
0<s<\frac{1}{2}
$$

Without loss of generality, we assume that $N_{1} \geq N_{2}$.

Case $1\left(N_{1} \sim N_{2} \sim N_{\max }\right)$.

Subcase 1.a $\left(N_{3} \ll N_{1}^{\frac{1}{2}}\right)$. By the bilinear Strichartz estimate and Lemma 2.6, we have

$$
\begin{aligned}
\text { LHS of (4.4) } & \lesssim N_{1}^{\sigma}\left\|z_{1, N_{1}} z_{3, N_{3}}\right\|_{L_{T, x}^{2}}\left\|z_{1, N_{2}}\right\|_{L_{T, x}^{5}}\left\|w_{N_{4}}\right\|_{L_{T, x}^{\frac{10}{3}}} \\
& \lesssim T^{0+} N_{1}^{\sigma-s-\frac{1}{2}+} N_{2}^{-s} N_{3}^{1-2 s-}\left\|\mathbf{P}_{N_{1}} \phi^{\omega}\right\|_{H^{s}}\left\|\langle\nabla\rangle^{s} z_{1, N_{2}}\right\|_{L_{T, x}^{5}}\left\|z_{3, N_{3}}\right\|_{Y_{T}^{2 s-}} \\
& \leq T^{0+} N_{1}^{\sigma-3 s+} C\left(\left\|z_{1}\right\|_{A_{5}^{s}(T)},\left\|z_{3}\right\|_{X_{T}^{2 s-}}\right) .
\end{aligned}
$$

Hence, we obtain (4.4), provided that $\sigma<3 s$.

Subcase 1.b $\left(N_{3} \gtrsim N_{1}^{\frac{1}{2}}\right)$. By Hölder's inequality and Lemma 2.6 we have

$$
\begin{aligned}
\text { LHS of (4.4) } & \lesssim N_{1}^{\sigma}\left\|z_{1, N_{1}}\right\|_{L_{T, x}^{5}}\left\|z_{1, N_{2}}\right\|_{L_{T, x}^{5}}\left\|z_{3, N_{3}}\right\|_{L_{T, x}^{\frac{10}{3}}}\left\|w_{N_{4}}\right\|_{L_{T, x}^{\frac{10}{3}}} \\
& \leq T^{0+} N_{1}^{\sigma-s} N_{2}^{-s} N_{3}^{-2 s+} C\left(\left\|z_{1}\right\|_{A_{5}^{s}(T)},\left\|z_{3}\right\|_{X_{T}^{2 s-}}\right) \\
& \leq T^{0+} N_{1}^{\sigma-3 s+} C\left(\left\|z_{1}\right\|_{A_{5}^{s}(T)},\left\|z_{3}\right\|_{X_{T}^{2 s-}}\right) .
\end{aligned}
$$

Hence, we obtain (4.4), provided that $\sigma<3 s$.

Case $2\left(N_{1} \sim N_{3} \sim N_{\max } \gg N_{2}\right)$.

Subcase 2.a $\left(N_{2} \ll N_{1}^{\frac{1}{2}}\right)$. By the bilinear Strichartz estimate and Lemma 2.6, we have

LHS of (4.4) $\lesssim N_{1}^{\sigma}\left\|z_{1, N_{1}}\right\|_{L_{T, x}^{5}}\left\|z_{1, N_{2}} z_{3, N_{3}}\right\|_{L_{T, x}^{2}}\left\|w_{N_{4}}\right\|_{L_{T, x}^{\frac{10}{3}}}$

$$
\begin{aligned}
& \lesssim T^{0+} N_{1}^{\sigma-s} N_{2}^{1-s-} N_{3}^{-2 s-\frac{1}{2}+}\left\|\langle\nabla\rangle^{s} z_{1, N_{1}}\right\|_{L_{T, x}^{5}}\left\|\mathbf{P}_{N_{2}} \phi^{\omega}\right\|_{H^{s}}\left\|z_{3, N_{3}}\right\|_{Y_{T}^{2 s-}} \\
& \leq T^{0+} N_{1}^{\sigma-\frac{7}{2} s+} C\left(\left\|z_{1}\right\|_{A_{5}^{s}(T)},\left\|z_{3}\right\|_{X_{T}^{2 s-}}\right) .
\end{aligned}
$$

Hence, we obtain (4.4), provided that $\sigma<\frac{7}{2} s$.

Subcase 2.b $\left(N_{2} \gtrsim N_{1}^{\frac{1}{2}}\right)$. By Hölder's inequality and Lemma 2.6. we have

$$
\begin{aligned}
\text { LHS of (4.4) } & \lesssim N_{1}^{\sigma}\left\|z_{1, N_{1}}\right\|_{L_{T, x}^{5}}\left\|z_{1, N_{2}}\right\|_{L_{T, x}^{5}}\left\|z_{3, N_{3}}\right\|_{L_{T, x}^{\frac{10}{3}}\left\|w_{N_{4}}\right\|_{L_{T, x}^{\frac{10}{3}}}} \\
& \leq T^{0+} N_{1}^{\sigma-s} N_{2}^{-s} N_{3}^{-2 s+} C\left(\left\|z_{1}\right\|_{A_{5}^{s}(T)},\left\|z_{3}\right\|_{X_{T}^{2 s-}}\right) \\
& \leq T^{0+} N_{1}^{\sigma-\frac{7}{2} s+} C\left(\left\|z_{1}\right\|_{A_{5}^{s}(T)},\left\|z_{3}\right\|_{X_{T}^{2 s-}}\right) .
\end{aligned}
$$

Hence, we obtain (4.4), provided that $\sigma<\frac{7}{2} s$. 
Case $3\left(N_{1} \sim N_{4} \sim N_{\max } \gg N_{2}, N_{3}\right)$.

Subcase 3.a $\left(N_{2}, N_{3} \ll N_{1}^{\frac{1}{2}}\right)$. By the bilinear Strichartz estimate, we have LHS of (4.4) $\lesssim N_{1}^{\sigma}\left\|z_{1, N_{1}} z_{1, N_{2}}\right\|_{L_{T, x}^{2}}\left\|z_{3, N_{3}} w_{N_{4}}\right\|_{L_{T, x}^{2}}$

$$
\begin{aligned}
& \lesssim T^{0+} N_{1}^{\sigma-s-\frac{1}{2}+} N_{2}^{1-s-} N_{3}^{1-2 s-} N_{4}^{-\frac{1}{2}+}\left(\prod_{j=1}^{2}\left\|\mathbf{P}_{N_{j}} \phi^{\omega}\right\|_{H^{s}}\right)\left\|z_{3, N_{3}}\right\|_{Y_{T}^{2 s-}}\left\|w_{N_{4}}\right\|_{Y_{T}^{0}} \\
& \leq T^{0+} N_{1}^{\sigma-\frac{5}{2} s+} C\left(\left\|z_{1}\right\|_{A_{5}^{s}(T)},\left\|z_{3}\right\|_{X_{T}^{2 s-}}\right) .
\end{aligned}
$$

Hence, we obtain (4.4), provided that $\sigma<\frac{5}{2} s$.

Subcase 3.b $\left(N_{2}, N_{3} \gtrsim N_{1}^{\frac{1}{2}}\right)$. By Hölder's inequality and Lemma 2.6, we have

$$
\begin{aligned}
\text { LHS of (4.4) } & \lesssim N_{1}^{\sigma}\left\|z_{1, N_{1}}\right\|_{L_{T, x}^{5}}\left\|z_{1, N_{2}}\right\|_{L_{T, x}^{5}}\left\|z_{3, N_{3}}\right\|_{L_{T, x}^{\frac{10}{3}}\left\|w_{N_{4}}\right\|_{L_{T, x}^{\frac{10}{3}}}} \\
& \leq T^{0+} N_{1}^{\sigma-s} N_{2}^{-s} N_{3}^{-2 s+} C\left(\left\|z_{1}\right\|_{A_{5}^{s}(T)},\left\|z_{3}\right\|_{X_{T}^{2 s-}}\right) \\
& \leq T^{0+} N_{1}^{\sigma-\frac{5}{2} s+} C\left(\left\|z_{1}\right\|_{A_{5}^{s}(T)},\left\|z_{3}\right\|_{X_{T}^{2 s-}}\right) .
\end{aligned}
$$

Hence, we obtain (4.4), provided that $\sigma<\frac{5}{2} s$.

Subcase 3.c $\left(N_{2} \gtrsim N_{1}^{\frac{1}{2}} \gg N_{3}\right)$. By the bilinear Strichartz estimate and Lemma 2.6. we have

$$
\begin{aligned}
\text { LHS of (4.4) } & \lesssim N_{1}^{\sigma}\left\|z_{1, N_{1}} z_{3, N_{3}}\right\|_{L_{T, x}^{2}}\left\|z_{1, N_{2}}\right\|_{L_{T, x}^{5}}\left\|w_{N_{4}}\right\|_{L_{T, x}^{\frac{10}{3}}} \\
& \leq T^{0+} N_{1}^{\sigma-s-\frac{1}{2}+} N_{2}^{-s} N_{3}^{1-2 s-} C\left(\left\|z_{1}\right\|_{A_{5}^{s}(T)},\left\|z_{3}\right\|_{X_{T}^{2 s-}}\right) \\
& \leq T^{0+} N_{1}^{\sigma-\frac{5}{2} s+} C\left(\left\|z_{1}\right\|_{A_{5}^{s}(T)},\left\|z_{3}\right\|_{X_{T}^{2 s-}}\right) .
\end{aligned}
$$

Hence, we obtain (4.4), provided that $\sigma<\frac{5}{2} s$.

Subcase 3.d $\left(N_{3} \gtrsim N_{1}^{\frac{1}{2}} \gg N_{2}\right)$. By the bilinear Strichartz estimate and Lemma 2.6. we have

$$
\begin{aligned}
\text { LHS of (4.4) } & \lesssim N_{1}^{\sigma}\left\|z_{1, N_{1}}\right\|_{L_{T, x}^{5}}\left\|z_{3, N_{3}}\right\|_{L_{T, x}^{\frac{10}{3}}\left\|z_{1, N_{2}} w_{N_{4}}\right\|_{L_{T, x}^{2}}} \\
& \leq T^{0+} N_{1}^{\sigma-s} N_{2}^{1-s-} N_{3}^{-2 s+} N_{4}^{-\frac{1}{2}+} C\left(\left\|z_{1}\right\|_{A_{5}^{s}(T)},\left\|z_{3}\right\|_{X_{T}^{2 s-}}\right) \\
& \leq T^{0+} N_{1}^{\sigma-\frac{5}{2} s+} C\left(\left\|z_{1}\right\|_{A_{5}^{s}(T)},\left\|z_{3}\right\|_{X_{T}^{2 s-}}\right) .
\end{aligned}
$$

Hence, we obtain (4.4), provided that $\sigma<\frac{5}{2} s$.

Case $4\left(N_{3} \sim N_{4} \gg N_{1} \geq N_{2}\right)$.

Subcase 4.a $\left(N_{1}, N_{2} \ll N_{3}^{\frac{1}{2}}\right)$. By the bilinear Strichartz estimate, we have

LHS of (4.4) $\lesssim N_{3}^{\sigma}\left\|z_{1, N_{1}} z_{3, N_{3}}\right\|_{L_{T, x}^{2}}\left\|z_{1, N_{2}} w_{N_{4}}\right\|_{L_{T, x}^{2}}$

$$
\begin{aligned}
& \lesssim T^{0+} N_{1}^{1-s-} N_{2}^{1-s-} N_{3}^{\sigma-2 s-\frac{1}{2}+} N_{4}^{-\frac{1}{2}+} C\left(\left\|z_{1}\right\|_{A_{5}^{s}(T)},\left\|z_{3}\right\|_{X_{T}^{2 s-}}\right) \\
& \leq T^{0+} N_{1}^{\sigma-3 s+} C\left(\left\|z_{1}\right\|_{A_{5}^{s}(T)},\left\|z_{3}\right\|_{X_{T}^{2 s-}}\right) .
\end{aligned}
$$

Hence, we obtain (4.4), provided that $\sigma<3 s$. 
Subcase 4.b $\left(N_{1}, N_{2} \gtrsim N_{3}^{\frac{1}{2}}\right)$. By Hölder's inequality and Lemma 2.6, we have

$$
\begin{aligned}
\text { LHS of (4.4) } & \lesssim N_{3}^{\sigma}\left\|z_{1, N_{1}}\right\|_{L_{T, x}^{5}}\left\|z_{1, N_{2}}\right\|_{L_{T, x}^{5}}\left\|z_{3, N_{3}}\right\|_{L_{T, x}^{\frac{10}{3}}\left\|w_{N_{4}}\right\|_{L_{T, x}^{\frac{10}{3}}}} \\
& \leq T^{0+} N_{1}^{-s} N_{2}^{-s} N_{3}^{\sigma-2 s+} C\left(\left\|z_{1}\right\|_{A_{5}^{s}(T)},\left\|z_{3}\right\|_{X_{T}^{2 s-}}\right) \\
& \leq T^{0+} N_{1}^{\sigma-3 s+} C\left(\left\|z_{1}\right\|_{A_{5}^{s}(T)},\left\|z_{3}\right\|_{X_{T}^{2 s-}}\right) .
\end{aligned}
$$

Hence, we obtain (4.4), provided that $\sigma<3 s$.

Subcase 4.c $\left(N_{1} \gtrsim N_{3}^{\frac{1}{2}} \gg N_{2}\right)$. By the bilinear Strichartz estimate and Lemma 2.6. we have

$$
\begin{aligned}
\text { LHS of (4.4) } & \lesssim N_{3}^{\sigma}\left\|z_{1, N_{2}}\right\|_{L_{T, x}^{5}}\left\|z_{1, N_{2}} z_{3, N_{3}}\right\|_{L_{T, x}^{2}}\left\|w_{N_{4}}\right\|_{L_{T, x}^{\frac{10}{3}}} \\
& \lesssim T^{0+} N_{1}^{-s} N_{2}^{1-s-} N_{3}^{\sigma-2 s-\frac{1}{2}+} C\left(\left\|z_{1}\right\|_{A_{5}^{s}(T)},\left\|z_{3}\right\|_{X_{T}^{2 s-}}\right) \\
& \leq T^{0+} N_{1}^{\sigma-3 s+} C\left(\left\|z_{1}\right\|_{A_{5}^{s}(T)},\left\|z_{3}\right\|_{X_{T}^{2 s-}}\right) .
\end{aligned}
$$

Hence, we obtain (4.4), provided that $\sigma<3 s$.

Putting all the cases together, we conclude that (4.4) holds, provided that $\sigma<$ $\frac{5}{2} s$. This completes the proof of Lemma 4.1.

4.2. On the fourth order term $z_{7}$. Next, we study the following fourth order term:

$$
z_{7}=-i \sum_{\substack{j_{1}+j_{2}+j_{3}=7 \\ j_{1}, j_{2}, j_{3} \in\{1,3,5\}}} \int_{0}^{t} S\left(t-t^{\prime}\right) z_{j_{1}} \overline{z_{j_{2}}} z_{j_{3}}\left(t^{\prime}\right) d t^{\prime} .
$$

In this case, there are two possibilities for $\left(j_{1}, j_{2}, j_{3}\right)$ in (4.5): $(1,3,3)$ and $(1,1,5)$ up to permutations. We denote by $\widetilde{z}_{7}$ the contribution to $z_{7}$ from $\left(j_{1}, j_{2}, j_{3}\right)=(1,3,3)$ (up to permutations). Note that the contribution to $z_{7}$ from $\left(j_{1}, j_{2}, j_{3}\right)=(1,1,5)$ (up to permutations) corresponds to $\zeta_{7}$ defined in (1.28). Dropping the complex conjugate, we have

$$
\begin{aligned}
& \widetilde{z}_{7} \sim \int_{0}^{t} S\left(t-t^{\prime}\right) z_{1} z_{3} z_{3}\left(t^{\prime}\right) d t^{\prime}, \\
& \zeta_{7} \sim \int_{0}^{t} S\left(t-t^{\prime}\right) z_{1} z_{1} z_{5}\left(t^{\prime}\right) d t^{\prime} .
\end{aligned}
$$

The following lemma shows that $\widetilde{z}_{7}$ and $\zeta_{7}$ enjoy a further gain of derivatives as compared to $z_{1}, z_{3}$, and $z_{5}$.

Lemma 4.2. Given $\phi \in H^{s}\left(\mathbb{R}^{3}\right)$, let $\phi^{\omega}$ be the Wiener randomization of $\phi$ defined in (1.3).

(i) Let $0<s<\frac{1}{2}$. Then, given any $\sigma<3 s$, we have

$$
\widetilde{z}_{7} \in X_{\mathrm{loc}}^{\sigma}
$$

almost surely.

(ii) Let $0<s<\frac{2}{5}$. Then, given any $\sigma<\frac{11}{4} s$, we have

$$
\zeta_{7} \in X_{\mathrm{loc}}^{\sigma} \text {, }
$$

almost surely. 
Proof. (i) We first estimate $\widetilde{z}_{7}$ in (4.6). Fix $0<s<\frac{1}{2}$. We proceed as in the proofs of Proposition 1.2 and Lemma 4.1. In view of Lemmas 2.1 and 2.2 and Proposition 1.2, it suffices to prove that there exists $\theta>0$ such that

$$
\begin{aligned}
\mid \int_{0}^{T} \int_{\mathbb{R}^{3}} N_{\max }^{\sigma} \mathbf{P}_{N_{1}} z_{1} \cdot \mathbf{P}_{N_{2}} z_{3} & \cdot \mathbf{P}_{N_{3}} z_{3} \cdot \mathbf{P}_{N_{4}} w d x d t \mid \\
& \leq T^{\theta} N_{\max }^{0-} C\left(\left\|z_{1}\right\|_{A_{7}^{s}(T)},\left\|z_{3}\right\|_{X_{T}^{2 s-}}\right)
\end{aligned}
$$

for all $N_{1}, \ldots, N_{4} \in 2^{\mathbb{N}_{0}}$ and $\|w\|_{Y_{T}^{0}} \leq 1$, where $A_{7}^{s}(T)$ is given by

$$
\left\|z_{1}\right\|_{A_{7}^{s}(T)}:=\max \left(\left\|\langle\nabla\rangle^{s} z_{1}\right\|_{L_{T, x}^{5}},\left\|\langle\nabla\rangle^{s} z_{1}\right\|_{L_{T}^{10+} L_{x}^{10}},\left\|\langle\nabla\rangle^{s} z_{1}\right\|_{L_{T}^{15} L_{x}^{\frac{15}{4}}},\left\|z_{1}(0)\right\|_{H^{s}}\right) .
$$

Without loss of generality, we assume that $N_{2} \geq N_{3}$.

Case $1\left(N_{1} \sim N_{2} \sim N_{\max }\right)$.

Subcase 1.a $\left(N_{3} \ll N_{1}^{\frac{1}{2}}\right)$. By the bilinear Strichartz estimate and Lemma 2.6, we have

$$
\begin{aligned}
\text { LHS of (4.9) } & \lesssim N_{1}^{\sigma}\left\|z_{1, N_{1}}\right\|_{L_{T, x}^{5}}\left\|z_{3, N_{2}} z_{3, N_{3}}\right\|_{L_{T, x}^{2}}\left\|w_{N_{4}}\right\|_{L_{T, x}^{\frac{10}{3}}} \\
& \lesssim T^{0+} N_{1}^{\sigma-s} N_{2}^{-2 s-\frac{1}{2}+} N_{3}^{1-2 s-}\left\|\langle\nabla\rangle^{s} z_{1, N_{1}}\right\|_{L_{T, x}^{5}}\left(\prod_{j=2}^{3}\left\|z_{3, N_{j}}\right\|_{Y_{T}^{2 s-}}\right) \\
& \leq T^{0+} N_{1}^{\sigma-4 s+} C\left(\left\|z_{1}\right\|_{A_{7}^{s}(T)},\left\|z_{3}\right\|_{X_{T}^{2 s-}}\right) .
\end{aligned}
$$

Hence, we obtain (4.9), provided that $\sigma<4 s$.

Subcase 1.b $\left(N_{3} \gtrsim N_{1}^{\frac{1}{2}}\right)$. By Hölder's inequality and Lemma 2.6, we have

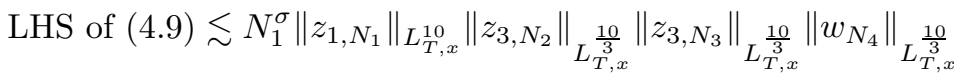

$$
\begin{aligned}
& \leq T^{0+} N_{1}^{\sigma-s} N_{2}^{-2 s+} N_{3}^{-2 s+} C\left(\left\|z_{1}\right\|_{A_{7}^{s}(T)},\left\|z_{3}\right\|_{X_{T}^{2 s-}}\right) \\
& \leq T^{0+} N_{1}^{\sigma-4 s+} C\left(\left\|z_{1}\right\|_{A_{7}^{s}(T)},\left\|z_{3}\right\|_{X_{T}^{2 s-}}\right) .
\end{aligned}
$$

Hence, we obtain (4.9), provided that $\sigma<4 s$.

Case $2\left(N_{1} \sim N_{4} \sim N_{\max } \gg N_{2} \geq N_{3}\right)$.

Subcase 2.a $\left(N_{2}, N_{3} \ll N_{1}^{\frac{1}{2}}\right)$. By the bilinear Strichartz estimate, we have

LHS of (4.9) $\lesssim N_{1}^{\sigma}\left\|z_{1, N_{1}} z_{3, N_{2}}\right\|_{L_{T, x}^{2}}\left\|z_{3, N_{3}} w_{N_{4}}\right\|_{L_{T, x}^{2}}$

$$
\begin{aligned}
& \lesssim T^{0+} N_{1}^{\sigma-s-\frac{1}{2}+} N_{2}^{1-2 s-} N_{3}^{1-2 s-} N_{4}^{-\frac{1}{2}+}\left\|\mathbf{P}_{N_{1}} \phi^{\omega}\right\|_{H^{s}}\left(\prod_{j=2}^{3}\left\|z_{3, N_{j}}\right\|_{Y_{T}^{2 s-}}\right) \\
& \leq T^{0+} N_{1}^{\sigma-3 s+} C\left(\left\|z_{1}\right\|_{A_{7}^{s}(T)},\left\|z_{3}\right\|_{X_{T}^{2 s-}}\right) .
\end{aligned}
$$

Hence, we obtain (4.9), provided that $\sigma<3 s$.

Subcase 2.b $\left(N_{2}, N_{3} \gtrsim N_{1}^{\frac{1}{2}}\right)$. By $L_{T, x}^{10}, L_{T, x}^{\frac{10}{3}}, L_{T, x}^{\frac{10}{3}}, L_{T, x}^{\frac{10}{3}}$-Hölder's inequality and Lemma 2.6. we have

$$
\begin{aligned}
\text { LHS of (4.9) } & \leq T^{0+} N_{1}^{\sigma-s} N_{2}^{-2 s+} N_{3}^{-2 s+} C\left(\left\|z_{1}\right\|_{A_{7}^{s}(T)},\left\|z_{3}\right\|_{X_{T}^{2 s-}}\right) \\
& \leq T^{0+} N_{1}^{\sigma-3 s+} C\left(\left\|z_{1}\right\|_{A_{7}^{s}(T)},\left\|z_{3}\right\|_{X_{T}^{2 s-}}\right) .
\end{aligned}
$$


Hence, we obtain (4.9), provided that $\sigma<3 s$.

Subcase 2.c $\left(N_{2} \gtrsim N_{1}^{\frac{1}{2}} \gg N_{3}\right)$. By the bilinear Strichartz estimate and Lemma 2.6. we have

$$
\begin{aligned}
\text { LHS of (4.9) } & \lesssim N_{1}^{\sigma}\left\|z_{1, N_{1}}\right\|_{L_{T, x}^{5}}\left\|z_{3, N_{2}}\right\|_{L_{T, x}^{\frac{10}{3}}}\left\|z_{3, N_{3}} w_{N_{4}}\right\|_{L_{T, x}^{2}} \\
& \leq T^{0+} N_{1}^{\sigma-s} N_{2}^{-2 s+} N_{3}^{1-2 s-} N_{4}^{-\frac{1}{2}+} C\left(\left\|z_{1}\right\|_{A_{7}^{s}(T)},\left\|z_{3}\right\|_{X_{T}^{2 s-}}\right) \\
& \leq T^{0+} N_{1}^{\sigma-3 s+} C\left(\left\|z_{1}\right\|_{A_{7}^{s}(T)},\left\|z_{3}\right\|_{X_{T}^{2 s-}}\right) .
\end{aligned}
$$

Hence, we obtain (4.9), provided that $\sigma<3 s$.

Case $3\left(N_{2} \sim N_{3} \sim N_{\max } \gg N_{1}\right)$.

Subcase 3.a $\left(N_{1} \ll N_{2}^{\frac{1}{2}}\right)$. By the bilinear Strichartz estimate and Lemma 2.6, we have

LHS of (4.9) $\lesssim N_{2}^{\sigma}\left\|z_{1, N_{1}} z_{3, N_{2}}\right\|_{L_{T, x}^{2}}\left\|z_{3, N_{3}}\right\|_{L_{T, x}^{5}}\left\|w_{N_{4}}\right\|_{L_{T, x}^{\frac{10}{3}}}$

$$
\lesssim T^{0+} N_{1}^{1-s-} N_{2}^{\sigma-2 s-\frac{1}{2}+} N_{3}^{-s}\left\|\mathbf{P}_{N_{1}} \phi^{\omega}\right\|_{H^{s}}\left\|z_{3, N_{2}}\right\|_{Y_{T}^{2 s-}}\left\|\langle\nabla\rangle^{s} z_{3, N_{3}}\right\|_{L_{T, x}^{5}} .
$$

By applying Lemma 3.3 and the fractional Leibniz rule,

$$
\begin{aligned}
& \lesssim T^{\frac{1}{10}+} N_{2}^{\sigma-\frac{7}{2} s+}\left\|\mathbf{P}_{N_{1}} \phi^{\omega}\right\|_{H^{s}}\left\|z_{3, N_{2}}\right\|_{Y_{T}^{2 s-}}\left\|\langle\nabla\rangle^{s} z_{1}\right\|_{L_{T}^{15} L_{x}^{\frac{15}{4}}}\left\|z_{1}\right\|_{L_{T}^{15} L_{x}^{\frac{15}{4}}}^{2} \\
& \leq T^{\frac{1}{10}+} N_{2}^{\sigma-\frac{7}{2} s+} C\left(\left\|z_{1}\right\|_{A_{7}^{s}(T)},\left\|z_{3}\right\|_{X_{T}^{2 s-}}\right) .
\end{aligned}
$$

Hence, we obtain (4.9), provided that $\sigma<\frac{7}{2} s$.

Subcase 3.b $\left(N_{1} \gtrsim N_{2}^{\frac{1}{2}}\right)$. By $L_{T, x}^{10}, L_{T, x}^{\frac{10}{3}}, L_{T, x}^{\frac{10}{3}}, L_{T, x}^{\frac{10}{3}}$-Hölder's inequality and Lemma 2.6, we have

$$
\begin{aligned}
\text { LHS of (4.9) } & \leq T^{0+} N_{1}^{-s} N_{2}^{\sigma-2 s+} N_{3}^{-2 s+} C\left(\left\|z_{1}\right\|_{A_{7}^{s}(T)},\left\|z_{3}\right\|_{X_{T}^{2 s-}}\right) \\
& \leq T^{0+} N_{1}^{\sigma-\frac{9}{2} s+} C\left(\left\|z_{1}\right\|_{A_{7}^{s}(T)},\left\|z_{3}\right\|_{X_{T}^{2 s-}}\right) .
\end{aligned}
$$

Hence, we obtain (4.9), provided that $\sigma<\frac{9}{2} s$.

Case $4\left(N_{2} \sim N_{4} \gg N_{1}, N_{3}\right)$.

Subcase 4.a $\left(N_{1}, N_{3} \ll N_{2}^{\frac{1}{2}}\right)$. By the bilinear Strichartz estimate, we have

LHS of (4.9) $\lesssim N_{2}^{\sigma}\left\|z_{1, N_{1}} z_{3, N_{2}}\right\|_{L_{T, x}^{2}}\left\|z_{3, N_{3}} w_{N_{4}}\right\|_{L_{T, x}^{2}}$

$$
\begin{aligned}
& \leq T^{0+} N_{1}^{1-s-} N_{2}^{\sigma-2 s-\frac{1}{2}+} N_{3}^{1-2 s-} N_{4}^{-\frac{1}{2}+} C\left(\left\|z_{1}\right\|_{A_{7}^{s}(T)},\left\|z_{3}\right\|_{X_{T}^{2 s-}}\right) \\
& \leq T^{0+} N_{1}^{\sigma-\frac{7}{2} s+} C\left(\left\|z_{1}\right\|_{A_{7}^{s}(T)},\left\|z_{3}\right\|_{X_{T}^{2 s-}}\right) .
\end{aligned}
$$

Hence, we obtain (4.9), provided that $\sigma<\frac{7}{2} s$.

Subcase 4.b $\left(N_{1}, N_{3} \gtrsim N_{2}^{\frac{1}{2}}\right)$. By $L_{T, x}^{10}, L_{T, x}^{\frac{10}{3}}, L_{T, x}^{\frac{10}{3}}, L_{T, x}^{\frac{10}{3}}$-Hölder's inequality and Lemma 2.6. we have

$$
\begin{aligned}
\text { LHS of (4.9) } & \leq T^{0+} N_{1}^{-s} N_{2}^{\sigma-2 s+} N_{3}^{-2 s+} C\left(\left\|z_{1}\right\|_{A_{7}^{s}(T)},\left\|z_{3}\right\|_{X_{T}^{2 s-}}\right) \\
& \leq T^{0+} N_{2}^{\sigma-\frac{7}{2} s+} C\left(\left\|z_{1}\right\|_{A_{7}^{s}(T)},\left\|z_{3}\right\|_{X_{T}^{2 s-}}\right) .
\end{aligned}
$$


Hence, we obtain (4.9), provided that $\sigma<\frac{7}{2} s$.

Subcase 4.c $\left(N_{1} \gtrsim N_{2}^{\frac{1}{2}} \gg N_{3}\right)$. By the bilinear Strichartz estimate and Lemma 2.6. we have

$$
\begin{aligned}
\text { LHS of (4.9) } & \lesssim N_{2}^{\sigma}\left\|z_{1, N_{1}}\right\|_{L_{T, x}^{5}}\left\|z_{3, N_{2}} z_{3, N_{3}}\right\|_{L_{T, x}^{2}}\left\|w_{N_{4}}\right\|_{L_{T, x}^{\frac{10}{3}}} \\
& \leq T^{0+} N_{1}^{-s} N_{2}^{\sigma-2 s-\frac{1}{2}+} N_{3}^{1-2 s-} C\left(\left\|z_{1}\right\|_{A_{7}^{s}(T)},\left\|z_{3}\right\|_{X_{T}^{2 s-}}\right) \\
& \leq T^{0+} N_{1}^{\sigma-\frac{7}{2} s+} C\left(\left\|z_{1}\right\|_{A_{7}^{s}(T)},\left\|z_{3}\right\|_{X_{T}^{2 s-}}\right) .
\end{aligned}
$$

Hence, we obtain (4.9), provided that $\sigma<\frac{7}{2} s$.

Subcase 4.d $\left(N_{3} \gtrsim N_{2}^{\frac{1}{2}} \gg N_{1}\right)$. By the bilinear Strichartz estimate and Lemma 2.6. we have

$$
\begin{aligned}
\text { LHS of (4.9) } & \lesssim N_{2}^{\sigma}\left\|z_{1, N_{1}} z_{3, N_{2}}\right\|_{L_{T, x}^{2}}\left\|z_{3, N_{3}}\right\|_{L_{T, x}^{5}}\left\|w_{N_{4}}\right\|_{L_{T, x}^{\frac{10}{3}}} \\
& \lesssim T^{0+} N_{1}^{1-s-} N_{2}^{\sigma-2 s-\frac{1}{2}+} N_{3}^{-s}\left\|\mathbf{P}_{N_{1}} \phi^{\omega}\right\|_{H^{s}}\left\|z_{3, N_{2}}\right\|_{Y_{T}^{2 s-}}\left\|\langle\nabla\rangle^{s} z_{3, N_{3}}\right\|_{L_{T, x}^{5}} .
\end{aligned}
$$

Proceeding as in Subcase 3.a with Lemma 3.3

$$
\leq T^{\frac{1}{10}} N_{1}^{\sigma-3 s+} C\left(\left\|z_{1}\right\|_{A_{7}^{s}(T)},\left\|z_{3}\right\|_{X_{T}^{2 s-}}\right) .
$$

Hence, we obtain (4.9), provided that $\sigma<3 s$.

Putting all the cases together, we conclude that (4.9) holds when $\sigma<3 s$.

(ii) Next, we estimate $\zeta_{7}$ in (4.7). This term has a similar structure to $z_{5}$ in (4.2); the only difference appears in the third factor. Hence, we can estimate $\zeta_{7}$ simply by replacing the regularity $2 s-$ (for $z_{3}$, see Proposition 1.2 ) with $\frac{5}{2} s-$ (for $z_{5}$, see Lemma 4.1) in the proof of Lemma 4.1. In the following, we only indicate the necessary modifications on the powers of dyadic parameters in the proof of Lemma 4.1 .

Case $1\left(N_{1} \sim N_{2} \sim N_{\max }\right)$.

Subcase 1.a $\left(N_{3} \ll N_{1}^{\frac{1}{2}}\right)$. It suffices to note that

$$
N_{1}^{\sigma-s-\frac{1}{2}+} N_{2}^{-s} N_{3}^{1-\frac{5}{2} s-} \lesssim N_{1}^{\sigma-\frac{13}{4} s+} \lesssim N_{\max }^{0-},
$$

provided that $\sigma<\frac{13}{4} s$ and $0<s<\frac{2}{5}$. The modification for Subcase 1.b is straightforward under the same regularity restriction.

Case $2\left(N_{1} \sim N_{3} \sim N_{\max } \gg N_{2}\right)$.

Subcase 2.a $\left(N_{2} \ll N_{1}^{\frac{1}{2}}\right)$. It suffices to note that

$$
N_{1}^{\sigma-s} N_{2}^{1-s-} N_{3}^{-\frac{5}{2} s-\frac{1}{2}+} \lesssim N_{1}^{\sigma-4 s+} \lesssim N_{\max }^{0-},
$$

provided that $\sigma<4 s$ and $0 \leq s<124$ The modification for Subcase 2.b is straightforward under the same regularity restriction.

Case $3\left(N_{1} \sim N_{4} \sim N_{\max } \gg N_{2}, N_{3}\right)$.

\footnotetext{
${ }^{24}$ The lower bound on $s$ is needed only for Subcase 2.b. Similar comments apply to the following cases and also to the proof of Proposition 1.7
} 
Subcase 3.a $\left(N_{2}, N_{3} \ll N_{1}^{\frac{1}{2}}\right)$. It suffices to note that

$$
N_{1}^{\sigma-s-\frac{1}{2}+} N_{2}^{1-s-} N_{3}^{1-\frac{5}{2} s-} N_{4}^{-\frac{1}{2}+} \lesssim N_{1}^{\sigma-\frac{11}{4} s+} \lesssim N_{\max }^{0-}
$$

provided that

$$
\sigma<\frac{11}{4} s \quad \text { and } \quad 0<s<\frac{2}{5} .
$$

The modifications for Subcases 3.b, 3.c, and 3.d are straightforward under the regularity restriction (4.10).

Case $4\left(N_{3} \sim N_{4} \gg N_{1} \geq N_{2}\right)$.

Subcase 4.a $\left(N_{1}, N_{2} \ll N_{3}^{\frac{1}{2}}\right)$. It suffices to note that

$$
N_{1}^{1-s-} N_{2}^{1-s-} N_{3}^{\sigma-\frac{5}{2} s-\frac{1}{2}+} N_{4}^{-\frac{1}{2}+} \lesssim N_{1}^{\sigma-\frac{7}{2} s+} \lesssim N_{\max }^{0-},
$$

provided that $\sigma<\frac{7}{2} s$ and $0 \leq s<1$. The modifications for Subcases 4.b and 4.c are straightforward under the same regularity restriction.

Putting all the cases together, we conclude that (4.8) holds under the regularity restriction (4.10). This completes the proof of Lemma 4.2 .

4.3. On the "unbalanced" higher order terms $\zeta_{2 k-1}$. In this subsection, we study the regularity properties of the unbalanced higher order terms $\zeta_{2 k-1}$ defined in (1.28). Note that $\left(j_{1}, j_{2}, j_{3}\right)=(1,1,2 k-3)$ up to permutations. Then, by dropping the complex conjugate, we have

$$
\zeta_{2 k-1} \sim \int_{0}^{t} S\left(t-t^{\prime}\right) z_{1} z_{1} \zeta_{2 k-3}\left(t^{\prime}\right) d t^{\prime}
$$

We first present the proof of Proposition 1.7

Proof of Proposition 1.7. We proceed by induction. From (1.29), we see that the recursive relation (1.30) is satisfied when $k=2,3,4$. In the following, by assuming

$$
\zeta_{2 k-3} \in X_{\mathrm{loc}}^{\sigma}
$$

for $\sigma<\alpha_{k-1} \cdot s$ almost surely, where $\alpha_{k-1}$ satisfies (1.32), we prove (1.30) and (1.31). As in the proof of Lemma 4.2(ii), the proof follows from the proof of Lemma 4.1 by replacing $z_{3}$ and $2 s-$ with $\zeta_{2 k-3}$ and $\alpha_{k-1} \cdot s-$, respectively. Therefore, we only indicate the necessary modifications on the powers of dyadic parameters in the proof of Lemma 4.1 .

Case $1\left(N_{1} \sim N_{2} \sim N_{\max }\right)$.

Subcase 1.a $\left(N_{3} \ll N_{1}^{\frac{1}{2}}\right)$. It suffices to note that

$$
N_{1}^{\sigma-s-\frac{1}{2}+} N_{2}^{-s} N_{3}^{1-\alpha_{k-1} s-} \lesssim N_{1}^{\sigma-\frac{\alpha_{k-1}+4}{2} s+} \lesssim N_{\max }^{0-},
$$

provided that

$$
\sigma<\frac{\alpha_{k-1}+4}{2} s \quad \text { and } \quad 0<s<\alpha_{k-1}^{-1} .
$$

The modification for Subcase 1.b is straightforward, giving the regularity restriction (4.12).

Case $2\left(N_{1} \sim N_{3} \sim N_{\max } \gg N_{2}\right)$. 
Subcase 2.a $\left(N_{2} \ll N_{1}^{\frac{1}{2}}\right)$. It suffices to note that

$$
N_{1}^{\sigma-s} N_{2}^{1-s-} N_{3}^{-\alpha_{k-1} s-\frac{1}{2}+} \lesssim N_{1}^{\sigma-\frac{2 \alpha_{k-1}+3}{2} s+} \lesssim N_{\max }^{0-},
$$

provided that

$$
\sigma<\frac{2 \alpha_{k-1}+3}{2} s \quad \text { and } \quad 0 \leq s<1 .
$$

The modification for Subcase 2.b is straightforward, giving the regularity restriction (4.13).

Case $3\left(N_{1} \sim N_{4} \sim N_{\max } \gg N_{2}, N_{3}\right)$.

Subcase 3.a $\left(N_{2}, N_{3} \ll N_{1}^{\frac{1}{2}}\right)$. It suffices to note that

$$
N_{1}^{\sigma-s-\frac{1}{2}+} N_{2}^{1-s-} N_{3}^{1-\alpha_{k-1} s-} N_{4}^{-\frac{1}{2}+} \lesssim N_{1}^{\sigma-\frac{\alpha_{k-1}+3}{2} s+} \lesssim N_{\max }^{0-},
$$

provided that

$$
\sigma<\frac{\alpha_{k-1}+3}{2} s \quad \text { and } \quad 0<s<\alpha_{k-1}^{-1} .
$$

The modifications for Subcases 3.b, 3.c, and 3.d are straightforward, giving the regularity restriction (4.14).

Case $4\left(N_{3} \sim N_{4} \gg N_{1} \geq N_{2}\right)$.

Subcase 4.a $\left(N_{1}, N_{2} \ll N_{3}^{\frac{1}{2}}\right)$. It suffices to note that

$$
N_{1}^{1-s-} N_{2}^{1-s-} N_{3}^{\sigma-\alpha_{k-1} s-\frac{1}{2}+} N_{4}^{-\frac{1}{2}+} \lesssim N_{1}^{\sigma-\left(\alpha_{k-1}+1\right) s+} \lesssim N_{\max }^{0-},
$$

provided that

$$
\sigma<\left(\alpha_{k-1}+1\right) s \quad \text { and } \quad 0 \leq s<1 .
$$

The modifications for Subcases 4.a and 4.b are straightforward, giving the regularity restriction (4.15).

Since $\alpha_{k-1}$ satisfies (1.32), we have $\alpha_{k-1} \geq 1$, which in turn implies that $\frac{\alpha_{k-1}+3}{2}$ $\leq \alpha_{k-1}+1$. Therefore, we conclude (1.30) and (1.31).

We conclude this section by proving a gain of space-time integrability for $\zeta_{2 k-1}$ analogous to Lemma 3.3

Lemma 4.3. Let $s \geq 0$. Given $\phi \in H^{s}\left(\mathbb{R}^{3}\right)$, let $\phi^{\omega}$ be its Wiener randomization defined in (1.3). Fix an integer $k \geq 3$. Then, for any finite $q, r>2$, there exist $\theta_{k}=\theta_{k}(q, r)>0, \delta_{k}=\delta_{k}(q, r) \in(0,1)$, and $1 \leq q_{k}<\infty$ such that

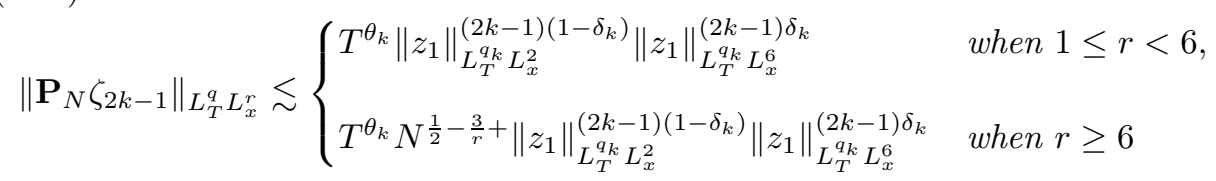

for any $T>0$ and $N \in 2^{\mathbb{N}_{0}}$. Note that the right-hand side of (4.16) is almost surely finite thanks to the probabilistic Strichartz estimate (Lemma 2.1). 
Proof. We prove (4.16) by induction. We first consider the case $r<6$. In Lemma 3.3. we proved (4.16) for $k=2$. Now, suppose that (4.16) holds for $k-1$. Then, from (4.11) and the dispersive estimate (3.10), we have

$$
\begin{aligned}
\left\|\mathbf{P}_{N} \zeta_{2 k-1}\right\|_{L_{T}^{q} L_{x}^{r}} & \leq\left\|\int_{0}^{t}\right\| \mathbf{P}_{N} S\left(t-t^{\prime}\right) z_{1}^{2} \zeta_{2 k-3}\left(t^{\prime}\right)\left\|_{L_{x}^{r}} d t^{\prime}\right\|_{L_{T}^{q}} \\
& \lesssim\left\|\int_{0}^{t} \frac{1}{\left|t-t^{\prime}\right|^{\frac{3}{2}-\frac{3}{r}}}\right\| z_{1}\left\|_{L_{x}^{3 r^{\prime}}}^{2}\right\| \zeta_{2 k-3}\left\|_{L_{x}^{3 r^{\prime}}} d t^{\prime}\right\|_{L_{T}^{q}} .
\end{aligned}
$$

Noting $3 r^{\prime}<6$ and applying the inductive hypothesis with $\theta_{k-1}=\theta_{k-1}\left(3 q, 3 r^{\prime}\right)$, $\delta_{k-1}=\delta_{k-1}\left(3 q, 3 r^{\prime}\right)$, and $q_{k-1}=q_{k-1}\left(3 q, 3 r^{\prime}\right)$,

$$
\lesssim T^{\frac{3}{r}-\frac{1}{2}+\theta_{k-1}}\left\|z_{1}\right\|_{L_{T}^{3 q} L_{x}^{3 r^{\prime}}}^{2}\left\|z_{1}\right\|_{L_{T}^{q_{k-1}} L_{x}^{2}}^{(2 k-3)\left(1-\delta_{k-1}\right)}\left\|z_{1}\right\|_{L_{T}^{q_{k-1}} L_{x}^{6}}^{(2 k-3) \delta_{k-1}} .
$$

By interpolation in $x$ and Hölder's inequality in $t$,

$$
\lesssim T^{\theta_{k}}\left\|z_{1}\right\|_{L_{T}^{q_{k}} L_{x}^{2}}^{(2 k-1)\left(1-\delta_{k}\right)}\left\|z_{1}\right\|_{L_{T}^{q_{k}} L_{x}^{6}}^{(2 k-1) \delta_{k}}
$$

When $r \geq 6$, (4.16) follows from Sobolev's inequality and (4.17) as in the proof of Lemma 3.3

\section{Proof of Theorem 1.3}

In this section, we study the fixed point problem (1.14) around the second order expansion (1.12) and present the proof of Theorem 1.3. Given $\frac{1}{2} \leq \sigma \leq 1$, let $\frac{2}{5} \sigma<s<\frac{1}{2}$. Given $\phi \in H^{s}\left(\mathbb{R}^{3}\right)$, let $\phi^{\omega}$ be its Wiener randomization defined in (1.3) and let $z_{1}$ and $z_{3}$ be as in (1.6) and (1.10). Define $\Gamma$ by

$$
\Gamma v(t)=-i \int_{0}^{t} S\left(t-t^{\prime}\right)\left\{\mathcal{N}\left(v+z_{1}+z_{3}\right)-\mathcal{N}\left(z_{1}\right)\right\}\left(t^{\prime}\right) d t^{\prime}
$$

Then, we have the following nonlinear estimates.

Proposition 5.1. Given $\frac{1}{2} \leq \sigma \leq 1$, let $\frac{2}{5} \sigma<s<\frac{1}{2}$. Then, there exist $\theta>0$, $C_{1}, C_{2}>0$, and an almost surely finite constant $R=R(\omega)>0$ such that

$$
\begin{gathered}
\|\Gamma v\|_{X^{\sigma}([0, T])} \leq C_{1}\left(\|v\|_{X^{\sigma}([0, T])}^{3}+T^{\theta} R(\omega)\right) \\
\left\|\Gamma v_{1}-\Gamma v_{2}\right\|_{X^{\sigma}([0, T])} \leq C_{2}\left(\sum_{j=1}^{2}\left\|v_{j}\right\|_{X^{\sigma}([0, T])}^{2}+T^{\theta} R(\omega)\right)\left\|v_{1}-v_{2}\right\|_{X^{\sigma}([0, T])}
\end{gathered}
$$

for all $v, v_{1}, v_{2} \in X^{\sigma}([0, T])$ and $0<T \leq 1$.

Once we prove Proposition 5.1, Theorem 1.3 immediately follows from a standard argument and thus we omit details. See Section 5 in [2].

Proof of Proposition 5.1. Let $0<T \leq 1$. We only prove (5.1) since (5.2) follows in a similar manner. Arguing as in the proof of Proposition 4.1 in [2, it suffices to perform a case-by-case analysis of expressions of the form

$$
\left|\int_{0}^{T} \int_{\mathbb{R}^{3}}\langle\nabla\rangle^{\sigma}\left(w_{1} w_{2} w_{3}\right) w d x d t\right|
$$

where $\|w\|_{Y_{T}^{0}} \leq 1$ and $w_{j}=v, z_{1}$, or $z_{3}, j=1,2,3$, but not all $z_{1}$. Note that we have dropped the complex conjugate sign on $w_{2}$ since it does not play any essential 
role. Then, we need to consider the following cases:
(A) $z_{1} z_{1} z_{3}$,
(D) $v v z_{1}$,
(G) $v z_{3} z_{3}$,
(B) $z_{1} z_{3} z_{3}$,
(E) $v v z_{3}$,
(H) $v z_{3} z_{1}$,
(C) $z_{3} z_{3} z_{3}$,
(F) $v z_{1} z_{1}$,
( I) $v v v$.

We already treated Cases (A) and (B) in Lemmas 4.1 and 4.2. In particular, Case (A) imposes the regularity restriction

$$
\sigma<\frac{5}{2} s
$$

As we see below, Cases (B) - (I) only impose a milder regularity restriction: $\sigma<3 s$.

Given $\frac{1}{2} \leq \sigma \leq 1$, let $\frac{2}{5} \sigma<s<\frac{1}{2}$. Define the $B^{s}(T)$-norm by

$$
\begin{aligned}
& \left\|z_{1}\right\|_{B^{s}(T)}:=\max \left(\left\|\langle\nabla\rangle^{s} z_{1}\right\|_{L_{T}^{5+} L_{x}^{5}},\left\|\langle\nabla\rangle^{s} z_{1}\right\|_{L_{T}^{10+} L_{x}^{10}},\right. \\
& \left.\left\|\langle\nabla\rangle^{s} z_{1}\right\|_{L_{T}^{3 q} L_{x}^{\frac{18}{5}+}},\left\|\langle\nabla\rangle^{s} z_{1}\right\|_{L_{T}^{15} L_{x}^{\frac{15}{4}}},\left\|z_{1}(0)\right\|_{H^{s}}\right),
\end{aligned}
$$

where $q \gg 1$ is defined in (5.4) below. Then, by applying the dyadic decomposition, we prove the following estimate 25

$$
\left|\int_{0}^{T} \int_{\mathbb{R}^{3}} N_{\max }^{\sigma}\left(\mathbf{P}_{N_{1}} w_{1} \mathbf{P}_{N_{2}} w_{2} \mathbf{P}_{N_{3}} w_{3}\right) \mathbf{P}_{N_{4}} w d x d t\right|
$$

$$
\leq C_{1} N_{\max }^{0-}\left(\|v\|_{X^{\sigma}([0, T])}^{3}+T^{\theta} C\left(\left\|z_{1}\right\|_{B^{s}(T)},\left\|z_{3}\right\|_{X^{2 s-}(T)}\right)\right)
$$

for all $N_{1}, \ldots, N_{4} \in 2^{\mathbb{N}_{0}}$. Once we prove (5.3), the desired estimate (5.1) follows from summing (5.3) over dyadic blocks and applying Lemmas 2.1 and 2.2 and Proposition 1.2

Case $\mathrm{C}\left(z_{3} z_{3} z_{3}\right.$ case). By symmetry, we assume $N_{1} \leq N_{2} \leq N_{3}$. Note that we have $N_{3} \sim N_{\text {max }}$.

Subcase C.1 $\left(N_{2} \sim N_{3} \sim N_{\max }\right)$. By Hölder's inequality with

$$
\left\{\begin{array}{l}
\text { time: } \frac{1}{q}+\frac{1}{2+}+\frac{1}{2+}+\frac{1}{\infty}=1 \text { for some } q \gg 1, \\
\text { space: } \frac{1}{6+}+\frac{1}{6-}+\frac{1}{6-}+\frac{1}{2}=1
\end{array}\right.
$$

such that $(2+, 6-)$ is admissible and applying Lemmas 2.6 and 3.3 , we have

$$
\begin{aligned}
& \text { LHS of }\left(\underline{5.3)} \lesssim N_{3}^{\sigma}\left\|z_{3, N_{1}}\right\|_{L_{T}^{q} L_{x}^{6+}}\left(\prod_{j=2}^{3}\left\|z_{3, N_{j}}\right\|_{L_{T}^{2+} L_{x}^{6-}}\right)\left\|w_{N_{4}}\right\|_{L_{T}^{\infty} L_{x}^{2}}\right. \\
& \qquad T^{0+} N_{1}^{-s+} N_{2}^{-2 s+} N_{3}^{\sigma-2 s+}\left\|\langle\nabla\rangle^{s} z_{1}\right\|_{L_{T}^{3 q} L_{x}^{\frac{18}{5}+}}\left\|z_{1}\right\|_{L_{T}^{3 q} L_{x}^{\frac{18}{5}+}}^{2} \\
& \quad \times\left(\prod_{j=2}^{3}\left\|z_{3, N_{j}}\right\|_{Y_{T}^{2 s-}}\right)\left\|w_{N_{4}}\right\|_{Y_{T}^{0}} \\
& \leq T^{0+} N_{3}^{\sigma-4 s+} C\left(\left\|z_{1}\right\|_{B^{s}(T)},\left\|z_{3}\right\|_{X_{T}^{2 s-}}\right) .
\end{aligned}
$$

\footnotetext{
${ }^{25}$ In Case (I), we do not perform the dyadic decomposition, and hence there is no need to have the factor $N_{\max }^{0-}$ on the right-hand side of (5.3).
} 
This yields (5.3), provided that $\sigma<4 s$ and $s>0$.

Subcase C.2 $\left(N_{3} \sim N_{4} \sim N_{\max } \gg N_{2} \geq N_{1}\right)$.

Subsubcase C.2.a $\left(N_{1}, N_{2} \ll N_{3}^{\frac{1}{2}}\right)$. By the bilinear Strichartz estimate, we have

LHS of (5.3) $\lesssim N_{3}^{\sigma}\left\|z_{3, N_{1}} z_{3, N_{3}}\right\|_{L_{T, x}^{2}}\left\|z_{3, N_{2}} w_{N_{4}}\right\|_{L_{T, x}^{2}}$

$$
\begin{aligned}
& \lesssim T^{0+} N_{1}^{1-2 s-} N_{2}^{1-2 s-} N_{3}^{\sigma-2 s-\frac{1}{2}+} N_{4}^{-\frac{1}{2}+}\left(\prod_{j=1}^{3}\left\|z_{3, N_{j}}\right\|_{Y_{T}^{2 s-}}\right) \\
& \lesssim T^{0+} N_{3}^{\sigma-4 s+} C\left(\left\|z_{3}\right\|_{X_{T}^{2 s-}}\right) .
\end{aligned}
$$

This yields (5.3), provided that $\sigma<4 s$ and $s<\frac{1}{2}$.

Subsubcase C.2.b $\left(N_{1}, N_{2} \gtrsim N_{3}^{\frac{1}{2}}\right)$. Proceeding as in Subcase (C.1), we have

$$
\begin{aligned}
& \text { LHS of (15.3) } \lesssim N_{3}^{\sigma}\left\|z_{3, N_{1}}\right\|_{L_{T}^{q} L_{x}^{6+}}\left(\prod_{j=2}^{3}\left\|z_{3, N_{2}}\right\|_{L_{T}^{2+} L_{x}^{6-}}\right)\left\|w_{N_{4}}\right\|_{L_{T}^{\infty} L_{x}^{2}} \\
& \quad \lesssim T^{0+} N_{1}^{-s+} N_{2}^{-2 s+} N_{3}^{\sigma-2 s+}\left\|\langle\nabla\rangle^{s} z_{1}\right\|_{L_{T}^{3 q} L_{x}^{\frac{18}{5}}+}\left\|z_{1}\right\|_{L_{T}^{3 q} L_{x}^{\frac{18}{5}+}}\left(\prod_{j=2}^{3}\left\|z_{3, N_{j}}\right\|_{Y_{T}^{2 s-}}\right) \\
& \quad \leq T^{0+} N_{3}^{\sigma-\frac{7}{2} s+} C\left(\left\|z_{1}\right\|_{B^{s}(T)},\left\|z_{3}\right\|_{X_{T}^{2 s-}}\right) .
\end{aligned}
$$

This yields (5.3), provided that $\sigma<\frac{7}{2} s$ and $s>0$.

Subsubcase C.2.c $\left(N_{2} \gtrsim N_{3}^{\frac{1}{2}} \gg N_{1}\right)$. By Lemmas 2.6 and 2.7 we have

LHS of (15.3) $\lesssim N_{1}^{\sigma}\left\|z_{3, N_{1}} z_{3, N_{3}}\right\|_{L_{T, x}^{2}}\left\|z_{3, N_{2}}\right\|_{L_{T, x}^{5}}\left\|w_{N_{4}}\right\|_{L_{T, x}^{\frac{10}{3}}}$

$$
\leq T^{0+} N_{1}^{1-2 s-} N_{2}^{-s} N_{3}^{\sigma-2 s-\frac{1}{2}+} C\left(\left\|z_{3}\right\|_{X_{T}^{2 s-}}\right)\left\|\langle\nabla\rangle^{s} z_{3, N_{2}}\right\|_{L_{T, x}^{5}} .
$$

By applying Lemma 3.3 .

$$
\begin{aligned}
& \leq T^{\frac{1}{10}+} N_{1}^{1-2 s-} N_{2}^{-s} N_{3}^{\sigma-2 s-\frac{1}{2}+} C\left(\left\|z_{3}\right\|_{X_{T}^{2 s-}}\right)\left\|\langle\nabla\rangle^{s} z_{1}\right\|_{L_{T}^{15} L_{x}^{\frac{15}{4}}\left\|z_{1}\right\|_{L_{T}^{15} L_{x}^{\frac{15}{4}}}^{2}} \leq T^{\frac{1}{10}+} N_{3}^{\sigma-\frac{7}{2} s+} C\left(\left\|z_{1}\right\|_{B^{s}(T)},\left\|z_{3}\right\|_{X_{T}^{2 s-}}\right) .
\end{aligned}
$$

This yields (5.3), provided that $\sigma<\frac{7}{2} s$ and $0 \leq s<\frac{1}{2}$.

Case D (vvz case). By symmetry, we assume $N_{1} \geq N_{2}$.

Subcase D.1 $\left(N_{1} \gtrsim N_{3}\right)$. In this case, we have $N_{1} \sim N_{\max }$.

Subsubcase D.1.a $\left(\max \left(N_{2}, N_{3}\right) \geq N_{1}^{\frac{1}{10}}\right)$. By Hölder's inequality and Lemma 2.6. we have

$$
\begin{aligned}
\text { LHS of (5.3) } & \lesssim N_{1}^{\sigma}\left\|v_{N_{1}}\right\|_{L_{T, x}^{\frac{10}{3}}\left\|v_{N_{2}}\right\|_{L_{T, x}^{\frac{10}{3}}}\left\|z_{1, N_{3}}\right\|_{L_{T, x}^{10}}\left\|w_{N_{4}}\right\|_{L_{T, x}^{\frac{10}{3}}}} \\
& \leq T^{0+} N_{2}^{-\sigma} N_{3}^{-s} C\left(\left\|z_{1}\right\|_{B^{s}(T)}\right)\|v\|_{X_{T}^{\sigma}}^{2} \\
& \leq T^{0+} N_{\max }^{0-} C\left(\left\|z_{1}\right\|_{B^{s}(T)}\right)\|v\|_{X_{T}^{\sigma}}^{2},
\end{aligned}
$$

provided that $\sigma>0$ and $s>0$. 
Subsubcase D.1.b $\left(\max \left(N_{2}, N_{3}\right) \ll N_{1}^{\frac{1}{10}}\right)$. In this case, we have $N_{1} \sim N_{4} \sim N_{\max }$. By Lemmas 2.6 and 2.7. we have

$$
\begin{aligned}
\text { LHS of (5.3) } & \lesssim N_{1}^{\sigma}\left\|v_{N_{1}}\right\|_{L_{T, x}^{\frac{10}{3}}}\left\|z_{1, N_{3}}\right\|_{L_{T, x}^{5}}\left\|v_{N_{2}} w_{N_{4}}\right\|_{L_{T, x}^{2}} \\
& \leq T^{0+} N_{2}^{1-\sigma-} N_{3}^{-s} N_{4}^{-\frac{1}{2}+} C\left(\left\|z_{1}\right\|_{B^{s}(T)}\right)\|v\|_{X_{T}^{\sigma}}^{2} \\
& \leq T^{0+} N_{\max }^{0-} C\left(\left\|z_{1}\right\|_{B^{s}(T)}\right)\|v\|_{X_{T}^{\sigma}}^{2},
\end{aligned}
$$

provided that $\sigma \geq 0$ and $s \geq 0$.

Subcase D.2 $\left(N_{3} \sim N_{4} \gg N_{1} \geq N_{2}\right)$.

Subsubcase D.2.a $\left(N_{1}, N_{2} \ll N_{3}^{\frac{1}{2}}\right)$. By the bilinear Strichartz estimate, we have

LHS of (5.3) $\lesssim N_{3}^{\sigma}\left\|v_{N_{1}} z_{1, N_{3}}\right\|_{L_{T, x}^{2}}\left\|v_{N_{2}} w_{N_{4}}\right\|_{L_{T, x}^{2}}$

$$
\begin{aligned}
& \lesssim T^{0+} N_{1}^{1-\sigma-} N_{2}^{1-\sigma-} N_{3}^{\sigma-s-\frac{1}{2}+} N_{4}^{-\frac{1}{2}+}\left(\prod_{j=1}^{2}\left\|v_{N_{j}}\right\|_{Y_{T}^{\sigma}}\right)\left\|\mathbf{P}_{N_{3}} \phi^{\omega}\right\|_{H^{s}} \\
& \leq T^{0+} N_{3}^{-s+} C\left(\left\|z_{1}\right\|_{B^{s}(T)}\right)\|v\|_{X_{T}^{\sigma}}^{2} \\
& \leq T^{0+} N_{\max }^{0-} C\left(\left\|z_{1}\right\|_{B^{s}(T)}\right)\|v\|_{X_{T}^{\sigma}}^{2},
\end{aligned}
$$

provided that $\sigma \leq 1$ and $s>0$.

Subsubcase D.2.b $\left(N_{1}, N_{2} \gtrsim N_{3}^{\frac{1}{2}}\right)$. Proceeding as in Subsubcase (D.1.a) with $L_{T, x}^{\frac{10}{3}}$, $L_{T, x}^{\frac{10}{3}}, L_{T, x}^{10}, L_{T, x}^{\frac{10}{3}}$-Hölder's inequality, it suffices to note that

$$
N_{1}^{-\sigma} N_{2}^{-\sigma} N_{3}^{\sigma-s} \lesssim N_{3}^{-s} \lesssim N_{\max }^{0-}
$$

provided that $\sigma \geq 0$ and $s>0$.

Subsubcase D.2.c $\left(N_{1} \gtrsim N_{3}^{\frac{1}{2}} \gg N_{2}\right)$. By Lemmas 2.6 and 2.7, we have

$$
\begin{aligned}
\text { LHS of (15.3) } & \lesssim N_{3}^{\sigma}\left\|v_{N_{1}}\right\|_{L_{T, x}^{\frac{10}{3}}\left\|z_{1, N_{3}}\right\|_{L_{T, x}^{5}}\left\|v_{N_{2}} w_{N_{4}}\right\|_{L_{T, x}^{2}}} \\
& \leq T^{0+} N_{1}^{-\sigma} N_{2}^{1-\sigma-} N_{3}^{\sigma-s} N_{4}^{-\frac{1}{2}+} C\left(\left\|z_{1}\right\|_{B^{s}(T)}\right)\|v\|_{X_{T}^{\sigma}}^{2} \\
& \leq T^{0+} N_{\max }^{0-} C\left(\left\|z_{1}\right\|_{B^{s}(T)}\right)\|v\|_{X_{T}^{\sigma}}^{2},
\end{aligned}
$$

provided that $0 \leq \sigma \leq 1$ and $s>0$.

Case E (vvz $z_{3}$ case). By symmetry, we assume $N_{1} \geq N_{2}$.

Subcase E.1 $\left(N_{1} \gtrsim N_{3}\right)$. In this case, we have $N_{1} \sim N_{\max }$.

Subsubcase E.1.a $\left(\max \left(N_{2}, N_{3}\right) \geq N_{1}^{\frac{1}{10}}\right)$. By Hölder's inequality with (5.4) as in Subcase (C.1) and Lemmas 2.6 and 3.3. we have

$$
\begin{aligned}
\text { LHS of (15.3) } & \lesssim N_{1}^{\sigma}\left(\prod_{j=1}^{2}\left\|v_{N_{j}}\right\|_{L_{T}^{2+} L_{x}^{6-}}\right)\left\|z_{3, N_{3}}\right\|_{L_{T}^{q} L_{x}^{6+}}\left\|w_{N_{4}}\right\|_{L_{T}^{\infty} L_{x}^{2}} \\
& \lesssim T^{0+} N_{2}^{-\sigma} N_{3}^{-s+}\|v\|_{X_{T}^{\sigma}}^{2}\left\|\langle\nabla\rangle^{s} z_{1}\right\|_{L_{T}^{3 q} L_{x}^{\frac{18}{5}}+\left\|z_{1}\right\|_{L_{T}^{3 q} L_{x}^{\frac{18}{5}}+}^{2}} \\
& \leq T^{0+} N_{\max }^{0-} C\left(\left\|z_{1}\right\|_{B^{s}(T)}\right)\|v\|_{X_{T}^{\sigma}}^{2},
\end{aligned}
$$

provided that $\sigma>0$ and $s>0$. 
Subsubcase E.1.b $\left(\max \left(N_{2}, N_{3}\right) \ll N_{1}^{\frac{1}{10}}\right)$. In this case, we have $N_{1} \sim N_{4} \sim N_{\max }$. By Lemmas 2.6, 2.7, and 3.3, we have

$$
\begin{aligned}
\text { LHS of (5.3) } & \lesssim N_{1}^{\sigma}\left\|v_{N_{1}}\right\|_{L_{T, x}^{\frac{10}{3}}\left\|z_{3, N_{3}}\right\|_{L_{T, x}^{5}}\left\|v_{N_{2}} w_{N_{4}}\right\|_{L_{T, x}^{2}}} \\
& \lesssim T^{\frac{1}{10}+} N_{2}^{1-\sigma-} N_{3}^{-s} N_{4}^{-\frac{1}{2}+}\|v\|_{X_{T}^{\sigma}}^{2}\left\|\langle\nabla\rangle^{s} z_{1}\right\|_{L_{T}^{15} L_{x}^{\frac{15}{4}}}\left\|z_{1}\right\|_{L_{T}^{15} L_{x}^{\frac{15}{4}}}^{2} \\
& \leq T^{\frac{1}{10}+} N_{\max }^{0-} C\left(\left\|z_{1}\right\|_{B^{s}(T)}\right)\|v\|_{X_{T}^{\sigma}}^{2},
\end{aligned}
$$

provided that $\sigma \geq 0$ and $s \geq 0$.

Subcase E.2 $\left(N_{3} \sim N_{4} \gg N_{1} \geq N_{2}\right)$.

Subsubcase E.2.a $\left(N_{1}, N_{2} \ll N_{3}^{\frac{1}{2}}\right)$. In this case, we proceed as in Subsubcase (D.2.a). It suffices to note that

$$
N_{1}^{1-\sigma-} N_{2}^{1-\sigma-} N_{3}^{\sigma-2 s-\frac{1}{2}+} N_{4}^{-\frac{1}{2}+} \lesssim N_{3}^{-2 s+} \lesssim N_{\max }^{0-},
$$

provided that $\sigma \leq 1$ and $s>0$.

Subsubcase E.2.b $\left(N_{1}, N_{2} \gtrsim N_{3}^{\frac{1}{2}}\right)$. By Hölder's inequality with (5.4) as in Subcase (C.1) and Lemmas 2.6 and 3.3, we have

$$
\begin{aligned}
& \text { LHS of }\left(\underline{5.31)} \lesssim N_{3}^{\sigma}\left(\prod_{j=1}^{2}\left\|v_{N_{j}}\right\|_{L_{T}^{2+} L_{x}^{6-}}\right)\left\|z_{3, N_{3}}\right\|_{L_{T}^{q} L_{x}^{6+}}\left\|w_{N_{4}}\right\|_{L_{T}^{\infty} L_{x}^{2}}\right.
\end{aligned}
$$

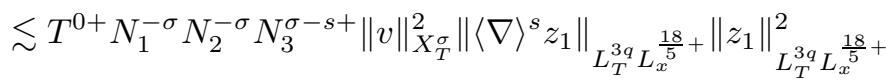

$$
\begin{aligned}
& \leq T^{0+} N_{\max }^{0-} C\left(\left\|z_{1}\right\|_{B^{s}(T)}\right)\|v\|_{X_{T}^{\sigma}}^{2},
\end{aligned}
$$

provided that $\sigma \geq 0$ and $s>0$.

Subsubcase E.2.c $\left(N_{1} \gtrsim N_{3}^{\frac{1}{2}} \gg N_{2}\right)$. By Lemmas 2.6, 2.7, and 3.3, we have

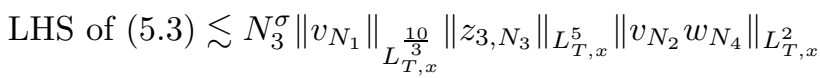

$$
\begin{aligned}
& \leq T^{\frac{1}{10}} N_{1}^{-\sigma} N_{2}^{1-\sigma-} N_{3}^{\sigma-s} N_{4}^{-\frac{1}{2}+}\|v\|_{X_{T}^{\sigma}}^{2}\left\|\langle\nabla\rangle^{s} z_{1}\right\|_{L_{T}^{15} L_{x}^{\frac{15}{4}}}\left\|z_{1}\right\|_{L_{T}^{15} \frac{15}{L_{x}^{4}}}^{2} \\
& \leq T^{\frac{1}{10}} N_{\max }^{0-} C\left(\left\|z_{1}\right\|_{B^{s}(T)}\right)\|v\|_{X_{T}^{\sigma}}^{2},
\end{aligned}
$$

provided that $0 \leq \sigma \leq 1$ and $s>0$.

Case $\mathrm{F}\left(v z_{1} z_{1}\right.$ case). By symmetry, we assume $N_{3} \geq N_{2}$.

Subcase F.1 $\left(N_{1} \gtrsim N_{3}\right)$. In this case, we have $N_{1} \sim N_{\max }$.

Subsubcase F.1.a $\left(\max \left(N_{2}, N_{3}\right) \geq N_{1}^{\frac{1}{10}}\right)$. By Lemma 2.6, we have

$$
\begin{aligned}
\text { LHS of (15.3) } & \lesssim N_{1}^{\sigma}\left\|v_{N_{1}}\right\|_{L_{T, x}^{\frac{10}{3}}\left\|z_{1, N_{2}}\right\|_{L_{T, x}^{5}}\left\|z_{1, N_{3}}\right\|_{L_{T, x}^{5}}\left\|w_{N_{4}}\right\|_{L_{T, x}^{\frac{10}{3}}}} \\
& \leq T^{0+} N_{2}^{-s} N_{3}^{-s} C\left(\left\|z_{1}\right\|_{B^{s}(T)}\right)\|v\|_{X_{T}^{\sigma}} \\
& \leq T^{0+} N_{\max }^{0-} C\left(\left\|z_{1}\right\|_{B^{s}(T)}\right)\|v\|_{X_{T}^{\sigma}},
\end{aligned}
$$

provided that $s>0$. 
Subsubcase F.1.b $\left(\max \left(N_{2}, N_{3}\right) \ll N_{1}^{\frac{1}{10}}\right)$. In this case, we proceed as in Subsubcase (D.1.b). It suffices to note that

$$
N_{2}^{1-s-} N_{3}^{-s} N_{4}^{-\frac{1}{2}+} \lesssim N_{\max }^{0-}
$$

provided that $s \geq 0$.

Subcase F.2 $\left(N_{2} \sim N_{3} \gg N_{1}\right)$.

Subsubcase F.2.a $\left(N_{1} \ll N_{3}^{\frac{1}{2}}\right)$. By Lemmas 2.6 and 2.7 we have

$$
\begin{aligned}
\text { LHS of (15.3) } & \lesssim N_{3}^{\sigma}\left\|v_{N_{1}} z_{1, N_{2}}\right\|_{L_{T, x}^{2}}\left\|z_{1, N_{3}}\right\|_{L_{T, x}^{5}}\left\|w_{N_{4}}\right\|_{L_{T, x}^{\frac{10}{3}}} \\
& \leq T^{0+} N_{1}^{1-\sigma-} N_{2}^{-s-\frac{1}{2}+} N_{3}^{\sigma-s} C\left(\left\|z_{1}\right\|_{B^{s}(T)}\right)\|v\|_{X_{T}^{\sigma}} \\
& \leq T^{0+} N_{3}^{\frac{\sigma}{2}-2 s+} C\left(\left\|z_{1}\right\|_{B^{s}(T)}\right)\|v\|_{X_{T}^{\sigma}} .
\end{aligned}
$$

This yields (5.3), provided that $\sigma \leq \min (1,4 s-)$.

Subsubcase F.2.b $\left(N_{1} \gtrsim N_{3}^{\frac{1}{2}}\right)$. In this case, we proceed with $L_{T, x}^{\frac{10}{3}}, L_{T, x}^{5}, L_{T, x}^{5}, L_{T, x^{-}}^{\frac{10}{3}}$ Hölder's inequality. It suffices to note that

$$
N_{1}^{-\sigma} N_{2}^{-s} N_{3}^{\sigma-s} \lesssim N_{3}^{\frac{\sigma}{2}-2 s} \lesssim N_{\max }^{0-}
$$

provided that $0 \leq \sigma<4 s$.

Subcase F.3 $\left(N_{3} \sim N_{4} \sim N_{\max } \gg N_{1}, N_{2}\right)$.

Subsubcase F.3.a $\left(N_{1}, N_{2} \ll N_{3}^{\frac{1}{2}}\right)$. By the bilinear Strichartz estimate, we have

LHS of (15.3) $\lesssim N_{3}^{\sigma}\left\|v_{N_{1}} z_{1, N_{3}}\right\|_{L_{T, x}^{2}}\left\|z_{1, N_{2}} w_{N_{4}}\right\|_{L_{T, x}^{2}}$

$$
\begin{aligned}
& \lesssim T^{0+} N_{1}^{1-\sigma-} N_{2}^{1-s-} N_{3}^{\sigma-s-\frac{1}{2}+} N_{4}^{-\frac{1}{2}+}\|v\|_{X_{T}^{\sigma}}\left(\prod_{j=2}^{3}\left\|\mathbf{P}_{N_{j}} \phi^{\omega}\right\|_{H^{s}}\right) \\
& \lesssim T^{0+} N_{3}^{\frac{\sigma}{2}-\frac{3}{2} s+} C\left(\left\|z_{1}\right\|_{B^{s}(T)}\right)\|v\|_{X_{T}^{\sigma}} .
\end{aligned}
$$

This yields (5.3), provided that $\sigma \leq \min (1,3 s-)$ and $s<1$.

Subsubcase F.3.b $\left(N_{1}, N_{2} \gtrsim N_{3}^{\frac{1}{2}}\right)$. In this case, we proceed with $L_{T, x}^{\frac{10}{3}}, L_{T, x}^{5}, L_{T, x}^{5}$, $L_{T, x}^{\frac{10}{3}}$-Hölder's inequality. It suffices to note that

$$
N_{1}^{-\sigma} N_{2}^{-s} N_{3}^{\sigma-s} \lesssim N_{3}^{\frac{\sigma}{2}-\frac{3}{2} s} \lesssim N_{\max }^{0-},
$$

provided that $0 \leq \sigma<3 s$.

Subsubcase F.3.c $\left(N_{1} \gtrsim N_{3}^{\frac{1}{2}} \gg N_{2}\right)$. By Lemmas 2.6 and 2.7, we have

$$
\begin{aligned}
\text { LHS of (5.3) } & \lesssim N_{3}^{\sigma}\left\|v_{N_{1}}\right\|_{L_{T, x}^{\frac{10}{3}}\left\|z_{1, N_{3}}\right\|_{L_{T, x}^{5}}\left\|z_{1, N_{2}} w_{N_{4}}\right\|_{L_{T, x}^{2}}} \\
& \leq T^{0+} N_{1}^{-\sigma} N_{2}^{1-s-} N_{3}^{\sigma-s} N_{4}^{-\frac{1}{2}+} C\left(\left\|z_{1}\right\|_{B^{s}(T)}\right)\|v\|_{X_{T}^{\sigma}} \\
& \leq T^{0+} N_{3}^{\frac{\sigma}{2}-\frac{3}{2} s+} C\left(\left\|z_{1}\right\|_{B^{s}(T)}\right)\|v\|_{X_{T}^{\sigma}} .
\end{aligned}
$$

This yields (5.3), provided that $0 \leq \sigma<3 s$ and $s<1$. 
Subsubcase F.3.d $\left(N_{2} \gtrsim N_{3}^{\frac{1}{2}} \gg N_{1}\right)$. By Lemmas 2.6 and 2.7, we have

$$
\begin{aligned}
\text { LHS of (5.3) } & \lesssim N_{3}^{\sigma}\left\|v_{N_{1}} z_{1, N_{3}}\right\|_{L_{T, x}^{2}}\left\|z_{1, N_{2}}\right\|_{L_{T, x}^{5}}\left\|w_{N_{4}}\right\|_{L_{T, x}^{\frac{10}{3}}} \\
& \lesssim T^{0+} N_{1}^{1-\sigma-} N_{2}^{-s} N_{3}^{\sigma-s-\frac{1}{2}+} C\left(\left\|z_{1}\right\|_{B^{s}(T)}\right)\|v\|_{X_{T}^{\sigma}} \\
& \leq T^{0+} N_{3}^{\frac{\sigma}{2}-\frac{3}{2} s+} C\left(\left\|z_{1}\right\|_{B^{s}(T)}\right)\|v\|_{X_{T}^{\sigma}} .
\end{aligned}
$$

This yields (5.3), provided that $\sigma \leq \min (1,3 s-)$ and $s \geq 0$.

Case G $\left(v z_{3} z_{3}\right.$ case). By symmetry, we assume that $N_{3} \geq N_{2}$.

Subcase G.1 $\left(N_{1} \gtrsim N_{3}\right)$. In this case, we have $N_{1} \sim N_{\max }$. We can proceed as in Subcase (E.1) with $v_{N_{2}}$ replaced by $z_{3, N_{2}}$. More precisely, when $\max \left(N_{2}, N_{3}\right) \geq$ $N_{1}^{\frac{1}{10}}$, we apply Hölder's inequality with (5.4) as in Subsubcase (E.1.a). It suffices to note that

$$
N_{2}^{-2 s+} N_{3}^{-s+} \lesssim N_{\max }^{0-}
$$

provided that $s>0$. When $\max \left(N_{2}, N_{3}\right) \ll N_{1}^{\frac{1}{10}}$, we proceed with Lemmas 2.6. 2.7. and 3.3 as in Subsubcase (E.1.b). In this case, it suffices to note that

$$
N_{2}^{1-2 s-} N_{3}^{-s} N_{4}^{-\frac{1}{2}+} \lesssim N_{\max }^{0-}
$$

provided that $s \geq 0$.

Subcase G.2 $\left(N_{2} \sim N_{3} \gg N_{1}\right)$.

Subsubcase G.2.a $\left(N_{1} \ll N_{3}^{\frac{1}{2}}\right)$. By Lemmas 2.6 and 2.7, we have

$$
\begin{aligned}
\text { LHS of } & \left(\overline{5.3)} \lesssim N_{3}^{\sigma}\left\|v_{N_{1}} z_{3, N_{2}}\right\|_{L_{T, x}^{2}}\left\|z_{3, N_{3}}\right\|_{L_{T, x}^{5}}\left\|w_{N_{4}}\right\|_{L_{T, x}^{\frac{10}{3}}}\right. \\
& \leq T^{\frac{1}{10}} N_{1}^{1-\sigma-} N_{2}^{-2 s-\frac{1}{2}+} N_{3}^{\sigma-s}\|v\|_{X_{T}^{\sigma}}\left\|z_{3, N_{2}}\right\|_{X_{T}^{2 s-}}\left\|\langle\nabla\rangle^{s} z_{1}\right\|_{L_{T}^{15} L_{x}^{\frac{15}{4}}}\left\|z_{1}\right\|_{L_{T}^{15} L_{x}^{\frac{15}{4}}}^{2} \\
& \leq T^{\frac{1}{10}} N_{3}^{\frac{\sigma}{2}-3 s+} C\left(\left\|z_{1}\right\|_{B^{s}(T)},\left\|z_{3}\right\|_{X_{T}^{2 s-}}\right)\|v\|_{X_{T}^{\sigma}} \\
& \leq T^{0+} N_{\max }^{0-} C\left(\left\|z_{1}\right\|_{B^{s}(T)},\left\|z_{3}\right\|_{X_{T}^{2 s-}}\right)\|v\|_{X_{T}^{\sigma}}
\end{aligned}
$$

provided that $\sigma \leq \min (1,6 s-)$.

Subsubcase G.2.b $\left(N_{1} \gtrsim N_{3}^{\frac{1}{2}}\right)$. In this case, we proceed with $L_{T, x}^{\frac{10}{3}}, L_{T, x}^{5}, L_{T, x}^{5}, L_{T, x^{-}}^{\frac{10}{3}}$ Hölder's inequality with Lemma 3.3. It suffices to note that

$$
N_{1}^{-\sigma} N_{2}^{-s} N_{3}^{\sigma-s} \lesssim N_{3}^{\frac{\sigma}{2}-2 s} \lesssim N_{\max }^{0-},
$$

provided that $0 \leq \sigma<4 s$.

Subcase G.3 $\left(N_{3} \sim N_{4} \sim N_{\max } \gg N_{1}, N_{2}\right)$.

Subsubcase G.3.a $\left(N_{1}, N_{2} \ll N_{3}^{\frac{1}{2}}\right)$. In this case, we proceed as in Subsubcase (F.3.a). It suffices to note that

$$
N_{1}^{1-\sigma-} N_{2}^{1-2 s-} N_{3}^{\sigma-2 s-\frac{1}{2}+} N_{4}^{-\frac{1}{2}+} \lesssim N_{3}^{\frac{\sigma}{2}-3 s+} \lesssim N_{\max }^{0-},
$$

provided that $\sigma \leq \min (1,6 s-)$ and $s<\frac{1}{2}$. 
Subsubcase G.3.b $\left(N_{1}, N_{2} \gtrsim N_{3}^{\frac{1}{2}}\right)$. In this case, we proceed with $L_{T, x}^{\frac{10}{3}}, L_{T, x}^{5}, L_{T, x}^{5}$, $L_{T, x}^{\frac{10}{3}}$-Hölder's inequality as in Subsubcase (F.3.b), but we apply Lemma 3.3 to estimate the $L_{T, x}^{5}$-norm of $z_{3, N_{j}}, j=2,3$. The regularity restriction is precisely the same as that in Subsubcase (F.3.b).

Subsubcase G.3.c $\left(N_{1} \gtrsim N_{3}^{\frac{1}{2}} \gg N_{2}\right)$. By Lemmas 2.6 2.7, and 3.3, we have

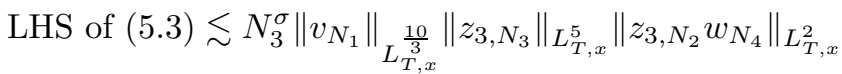

$$
\begin{aligned}
& \lesssim T^{\frac{1}{10}+} N_{1}^{-\sigma} N_{2}^{1-2 s-} N_{3}^{\sigma-s} N_{4}^{-\frac{1}{2}+}\|v\|_{X_{T}^{\sigma}}\left\|z_{3, N_{2}}\right\|_{X_{T}^{2 s-}}\left\|\langle\nabla\rangle^{s} z_{1}\right\|_{L_{T}^{15} L_{x}^{\frac{15}{4}}}\left\|z_{1}\right\|_{L_{T}^{15} L_{x}^{\frac{15}{4}}}^{2} \\
& \leq T^{\frac{1}{10}+} N_{3}^{\frac{\sigma}{2}-2 s+} C\left(\left\|z_{1}\right\|_{B^{s}(T)},\left\|z_{3}\right\|_{X_{T}^{2 s-}}\right)\|v\|_{X_{T}^{\sigma}} .
\end{aligned}
$$

This yields (5.3), provided that $0 \leq \sigma<4 s$ and $s<\frac{1}{2}$.

Subcase G.4 $\left(N_{2} \gtrsim N_{3}^{\frac{1}{2}} \gg N_{1}\right)$. By Lemmas 2.6, 2.7, and 3.3, we have

LHS of (5.3) $\lesssim N_{3}^{\sigma}\left\|v_{N_{1}} z_{3, N_{3}}\right\|_{L_{T, x}^{2}}\left\|z_{3, N_{2}}\right\|_{L_{T, x}^{5}}\left\|w_{N_{4}}\right\|_{L_{T, x}^{\frac{10}{3}}}$

$$
\begin{aligned}
& \lesssim T^{\frac{1}{10}+} N_{1}^{1-\sigma-} N_{2}^{-s} N_{3}^{\sigma-2 s-\frac{1}{2}+}\|v\|_{X_{T}^{\sigma}}\left\|\langle\nabla\rangle^{s} z_{1}\right\|_{L_{T}^{15} L_{x}^{\frac{15}{4}}}\left\|z_{1}\right\|_{L_{T}^{15} L_{x}^{\frac{15}{4}}}^{2}\left\|z_{3, N_{3}}\right\|_{X_{T}^{2 s-}} \\
& \leq T^{\frac{1}{10}+} N_{1}^{\frac{\sigma}{2}-\frac{5}{2} s+} C\left(\left\|z_{1}\right\|_{B^{s}(T)},\left\|z_{3}\right\|_{X_{T}^{2 s-}}\right)\|v\|_{X_{T}^{\sigma}} .
\end{aligned}
$$

This yields (5.3), provided that $\sigma \leq \min (1,5 s-)$ and $s \geq 0$.

Case $\mathrm{H}\left(v z_{3} z_{1}\right.$ case) .

Subcase H.1 $\left(N_{1} \sim N_{\max }\right)$. In this case, we can proceed as in Subcase (D.1) by replacing $v_{N_{2}}$ with $z_{3, N_{2}}$. When $\max \left(N_{2}, N_{3}\right) \geq N_{1}^{\frac{1}{10}}$, it suffices to note that

$$
N_{2}^{-2 s+} N_{3}^{-s} \lesssim N_{\max }^{0-}
$$

provided that $s>0$. When $\max \left(N_{2}, N_{3}\right) \ll N_{1}^{\frac{1}{10}}$, it suffices to note that

$$
N_{2}^{1-2 s-} N_{3}^{-s} N_{4}^{-\frac{1}{2}+} \lesssim N_{\max }^{0-}
$$

provided that $s \geq 0$.

Subcase H.2 $\left(N_{3} \sim N_{\max } \gg N_{1}\right)$.

Subsubcase H.2.a $\left(N_{1}, N_{2} \ll N_{3}^{\frac{1}{2}}\right)$. In this case, we have $N_{3} \sim N_{4} \sim N_{\max }$. Then, proceeding as in Subsubcase (F.3.a), it suffices to note that

$$
N_{1}^{1-\sigma-} N_{2}^{1-2 s-} N_{3}^{\sigma-s-\frac{1}{2}+} N_{4}^{-\frac{1}{2}+} \lesssim N_{3}^{\frac{\sigma}{2}-2 s} \lesssim N_{\max }^{0-},
$$

provided that $\sigma \leq \min (1,4 s-)$ and $s<\frac{1}{2}$.

Subsubcase H.2.b $\left(N_{1}, N_{2} \gtrsim N_{3}^{\frac{1}{2}}\right)$. In this case, we proceed with $L_{T, x}^{\frac{10}{3}}, L_{T, x}^{\frac{10}{3}}, L_{T, x}^{10}$, $L_{T, x}^{\frac{10}{3}}$-Hölder's inequality. It suffices to note that

$$
N_{1}^{-\sigma} N_{2}^{-2 s+} N_{3}^{\sigma-s} \lesssim N_{3}^{\frac{\sigma}{2}-2 s+} \lesssim N_{\max }^{0-}
$$

provided that $0 \leq \sigma<4 s$. 
Subsubcase H.2.c $\left(N_{1} \gtrsim N_{3}^{\frac{1}{2}} \gg N_{2}\right)$. In this case, we have $N_{3} \sim N_{4} \sim N_{\max }$. We can proceed as in Subsubcase (G.3.c) with $z_{3, N_{3}}$ replaced by $z_{1, N_{3}}$ (without applying Lemma [3.3). The regularity restriction is precisely the same as in Subsubcase (G.3.c).

Subsubcase H.2.d $\left(N_{2} \gtrsim N_{3}^{\frac{1}{2}} \gg N_{1}\right)$. We first consider the case $N_{3} \sim N_{4} \sim N_{\max }$. By Lemmas 2.6 and 2.7, we have

$$
\begin{aligned}
\text { LHS of (5.3) } & \lesssim N_{3}^{\sigma}\left\|v_{N_{1}} w_{N_{4}}\right\|_{L_{T, x}^{2}}\left\|z_{3, N_{2}}\right\|_{L_{T, x}^{\frac{10}{3}}}\left\|z_{1, N_{3}}\right\|_{L_{T, x}^{5}} \\
& \lesssim T^{0+} N_{1}^{1-\sigma-} N_{2}^{-2 s+} N_{3}^{\sigma-s} N_{4}^{-\frac{1}{2}+} C\left(\left\|z_{1}\right\|_{B^{s}(T)},\left\|z_{3}\right\|_{X_{T}^{2 s-}}\right)\|v\|_{X_{T}^{\sigma}} \\
& \leq T^{0+} N_{3}^{\frac{\sigma}{2}-2 s+} C\left(\left\|z_{1}\right\|_{B^{s}(T)},\left\|z_{3}\right\|_{X_{T}^{2 s-}}\right)\|v\|_{X_{T}^{\sigma}} .
\end{aligned}
$$

This yields (5.3), provided that $\sigma \leq \min (1,4 s-)$ and $s>0$.

When $N_{4} \ll N_{3}$, we have $N_{2} \sim N_{3} \sim N_{\max }$. In this case, we can repeat the computation above with the roles of $z_{3, N_{2}}$ and $w_{N_{4}}$ switched. Noting that

$$
N_{1}^{1-\sigma-} N_{2}^{-2 s-\frac{1}{2}+} N_{3}^{\sigma-s} \lesssim N_{3}^{\frac{\sigma}{2}-3 s+} \lesssim N_{\max }^{0-},
$$

we obtain (5.3), provided that $\sigma \leq \min (1,6 s-)$.

Subcase H.3 $\left(N_{2} \sim N_{4} \gg N_{1}, N_{3}\right)$.

Subsubcase H.3.a $\left(N_{1} \ll N_{2}^{\frac{1}{2}}\right)$. In this case, we can proceed as in Subsubcase (H.2.d). It suffices to note that

$$
N_{1}^{1-\sigma-} N_{2}^{\sigma-2 s+} N_{3}^{-s} N_{4}^{-\frac{1}{2}+} \lesssim N_{2}^{\frac{\sigma}{2}-2 s+} \lesssim N_{\max }^{0-},
$$

provided that $\sigma \leq \min (1,4 s-)$ and $s \geq 0$.

Subsubcase H.3.b $\left(N_{1} \gtrsim N_{2}^{\frac{1}{2}}\right)$. In this case, we proceed with $L_{T, x}^{\frac{10}{3}}, L_{T, x}^{\frac{10}{3}}, L_{T, x}^{10}, L_{T, x^{-}}^{\frac{10}{3}}$ Hölder's inequality. It suffices to note that

$$
N_{1}^{-\sigma} N_{2}^{\sigma-2 s+} N_{3}^{-s} \lesssim N_{2}^{\frac{\sigma}{2}-2 s+} \lesssim N_{\max }^{0-},
$$

provided that $0 \leq \sigma<4 s$.

Case I ( $v v v$ case). In this case, there is no need to perform the dyadic decomposition. By Hölder's inequality, Sobolev's inequality (2.3), and Lemma 2.6, we have

$$
\begin{aligned}
\text { LHS of (15.3) } & \lesssim\|v\|_{L_{T, x}^{5}}^{2}\left\|\langle\nabla\rangle^{\sigma} v\right\|_{L_{T, x}^{\frac{10}{3}}\|w\|_{L_{T, x}^{\frac{10}{3}}}} \\
& \lesssim\left\|\langle\nabla\rangle^{\frac{1}{2}} v\right\|_{L_{T}^{5} L_{x}^{\frac{30}{11}}}^{2}\left\|\langle\nabla\rangle^{\sigma} v\right\|_{L_{T, x}^{\frac{10}{3}}} \\
& \lesssim\|v\|_{X_{T}^{\sigma}}^{3},
\end{aligned}
$$

provided that $\sigma \geq \frac{1}{2}$.

Putting together all the cases, including Cases (A) and (B) treated in Lemmas 4.1 and 4.2 , we conclude that (5.3) holds, provided that $\frac{1}{2} \leq \sigma \leq 1$ and $\frac{2}{5} \sigma<s<\frac{1}{2}$. This completes the proof of Proposition 5.1 


\section{Proof of Theorem 1.8}

In this section, we briefly discuss the proof of Theorem 1.8. Given $\frac{1}{6}<s<\frac{1}{2}$, let $k \in \mathbb{N}$ such that $\frac{1}{2 \alpha_{k+1}}<s \leq \frac{1}{2 \alpha_{k}}$, where $\alpha_{k}$ is defined in (1.32). Our main goal is to study the fixed point problem (1.34). Define $\widetilde{\Gamma}$ by

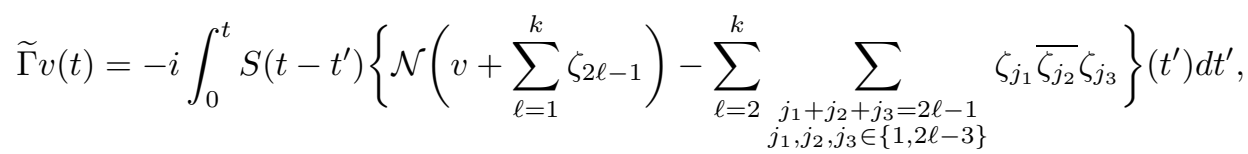

where $\zeta_{2 \ell-1}, \ell=1, \ldots, k$, is as in (1.28). Theorem 1.8 follows from a standard fixed point argument once we prove the following proposition.

Proposition 6.1. Given $\frac{1}{6}<s<\frac{1}{2}$, let $k \in \mathbb{N}$ such that $\frac{1}{2 \alpha_{k+1}}<s<\frac{1}{\alpha_{k}}$. Then, there exist $\theta>0, C_{1}, C_{2}>0$, and an almost surely finite constant $R=R(\omega)>0$ such that

$$
\begin{gathered}
\|\widetilde{\Gamma} v\|_{X^{\frac{1}{2}([0, T])}} \leq C_{1}\left(\|v\|_{X^{\frac{1}{2}([0, T])}}^{3}+T^{\theta} R(\omega)\right) \\
\left\|\widetilde{\Gamma} v_{1}-\widetilde{\Gamma} v_{2}\right\|_{X^{\frac{1}{2}([0, T])}} \leq C_{2}\left(\sum_{j=1}^{2}\left\|v_{j}\right\|_{X^{\frac{1}{2}([0, T])}}^{2}+T^{\theta} R(\omega)\right)\left\|v_{1}-v_{2}\right\|_{X^{\frac{1}{2}}([0, T])}
\end{gathered}
$$

for all $v, v_{1}, v_{2} \in X^{\frac{1}{2}}([0, T])$ and $0<T \leq 1$.

On the one hand, the upper bound $\frac{1}{\alpha_{k}}$ on the range of $s$ in Proposition 6.1 comes from the restriction in Proposition 1.7. On the other hand, given $\frac{1}{6}<s<\frac{1}{2}$, we fix $k \in \mathbb{N}$ such that $\frac{1}{2 \alpha_{k+1}}<s \leq \frac{1}{2 \alpha_{k}}$ and apply Proposition 6.1. Namely, the upper bound on $s$ in Proposition 6.1 does not cause any problem in proving Theorem 1.8 .

Proof. We only discuss the proof of (6.1) since (6.2) follows in a similar manner. As in the proof of Proposition 5.1, it suffices to perform a case-by-case analysis of expressions of the form

$$
\left|\int_{0}^{T} \int_{\mathbb{R}^{3}}\langle\nabla\rangle^{\frac{1}{2}}\left(w_{1} w_{2} w_{3}\right) w d x d t\right|
$$

where $\|w\|_{Y_{T}^{0}} \leq 1$ and $w_{j}=v, \zeta_{2 \ell-1}, \ell=1, \ldots, k$, but not of the form $\zeta_{1} \zeta_{1} \zeta_{2 \ell-3}$ for $\ell \in\{2,3, \ldots, k\}$. Here, we have dropped the complex conjugate sign on $w_{2}$ since it does not play any role in our analysis. More concretely, we need to consider the following cases:
(A) $\zeta_{1} \zeta_{1} \zeta_{2 k-1}$,
(D) $v v \zeta_{1}$,
(G) $v \zeta_{j_{2}} \zeta_{j_{3}}$,
(B) $\zeta_{1} \zeta_{j_{2}} \zeta_{j_{3}}$,
(E) $v v \zeta_{j_{3}}$,
(H) $v \zeta_{j_{2}} \zeta_{1}$,
(C) $\zeta_{j_{1}} \zeta_{j_{2}} \zeta_{j_{3}}$,
(F) $v \zeta_{1} \zeta_{1}$,
( I) $v v v$,

where $j_{1}, j_{2}, j_{3}$ can take any value in $\{3,5, \ldots, 2 k-1\}$. As we see below, the worst interaction appears in Case (A), and all the other cases can be handled as in the proof of Proposition 5.1 .

We first point out that, in the proof of Proposition 5.1 the regularity restriction coming from Cases (B) - (I) (with $\sigma=\frac{1}{2}$ ) is $s>\frac{1}{6}$. Moreover, by comparing Proposition 1.2 and Lemma 3.3 with Proposition 1.7 and Lemma 4.3 , we see that the unbalanced higher order term $\zeta_{2 \ell-1}$ for $\ell \in\{2,3, \ldots, k\}$ enjoys (at least) the 
same regularity properties as $z_{3}=\zeta_{3}$ both in terms of differentiability and spacetime integrability. Therefore, we can simply apply the estimates in the proofs of Lemma 4.2(i) for Case (B) and Proposition 5.1 for Cases (C) - (I) and conclude that the contributions from Cases (B) - (I) can be bounded, provided $s>\frac{1}{6}$.

It remains to consider Case (A). In view of (1.27), the contribution from Case (A) is nothing but $\zeta_{2 k+1}$. Hence, by applying Proposition 1.7 with $\sigma=\frac{1}{2}$, we conclude that the contribution in Case (A) can be estimated by $T^{\theta} R(\omega)$, provided that $\frac{1}{2 \alpha_{k+1}}<s<\frac{1}{\alpha_{k}}$. This completes the proof of Proposition 6.1

\section{Appendix A. On the Deterministic non-Smoothing of the Duhamel INTEGRAL OPERATOR}

In this appendix, we show that there is no deterministic smoothing on the Duhamel integral operator $\mathcal{I}$ defined in (1.17) when $s \leq \frac{1}{2}$.

Lemma A.1. Let $\sigma>3 s-1$. Then, the estimate (1.18) does not hold. In particular, there is no deterministic nonlinear smoothing when $s \leq \frac{1}{2}$.

Proof. Given $N \geq L \geq \ell \gg 1$, consider $u_{j}=S(t) \phi_{j}, j=1,2,3$, such that $\widehat{\phi}_{j}(\xi) \sim$ $\mathbf{1}_{A_{j}}(\xi)$, where

$$
A_{1}=L e_{1}+\ell Q, \quad A_{2}=\ell Q, \quad \text { and } \quad A_{3}=N e_{2}+\ell Q .
$$

Then, we have

$$
\prod_{j=1}^{3}\left\|u_{j}(0)\right\|_{H^{s}} \sim \ell^{\frac{9}{2}+s} L^{s} N^{s} .
$$

Let $A=L e_{1}+N e_{2}+\ell Q$. By writing

$$
\mathbf{1}_{A}(\xi) \mathcal{F}_{x}\left[\mathcal{I}\left(u_{1}, u_{2}, u_{3}\right)\right](t, \xi)
$$

$$
=-i \mathbf{1}_{A}(\xi) e^{-i t|\xi|^{2}} \int_{0}^{t} \int_{\xi=\xi_{1}-\xi_{2}+\xi_{3}} e^{i t^{\prime} \Phi(\bar{\xi})} \prod_{j=1}^{3} \mathbf{1}_{A_{j}}\left(\xi_{j}\right) d \xi_{1} d \xi_{2} d t^{\prime},
$$

we see that the non-trivial contribution to (A.2) comes from $\xi_{j} \in A_{j}, j=1,2,3$. Moreover, in this case, $\Phi(\bar{\xi})$, defined in (3.7), satisfies $|\Phi(\bar{\xi})| \lesssim N L$. In particular, by choosing

$$
t_{*} \sim \frac{1}{N L}
$$

such that $t_{*}|\Phi(\bar{\xi})| \ll 1$, we have (3.9) for all $t^{\prime} \in\left[0, t_{*}\right]$. Hence, by Lemma 3.1 with (A.3), we obtain

(A.4) $\left\|\mathcal{I}\left(u_{1}, u_{2}, u_{3}\right)\right\|_{X^{\sigma}([0,1])} \gtrsim\left\|\mathcal{I}\left(u_{1}, u_{2}, u_{3}\right)\left(t_{*}\right)\right\|_{H^{\sigma}} \gtrsim t_{*} \ell^{\frac{15}{2}} N^{\sigma} \sim \ell^{\frac{15}{2}} L^{-1} N^{\sigma-1}$.

Therefore, it follows from (A.1) and (A.4) that by choosing $\ell \sim L \sim N$, we have

$$
\frac{\left\|\mathcal{I}\left(u_{1}, u_{2}, u_{3}\right)\right\|_{X^{\sigma}([0,1])}}{\prod_{j=1}^{3}\left\|u_{j}(0)\right\|_{H^{s}}} \gtrsim \ell^{3-s} L^{-1-s} N^{\sigma-1-s} \sim N^{\sigma-3 s+1} \longrightarrow \infty,
$$

as $N \rightarrow \infty$, provided that $\sigma>3 s-1$. This shows that the estimate (1.18) cannot hold when $\sigma>3 s-1$. 
Define the quintilinear operator $\mathcal{I}^{(2)}$ by

$$
\mathcal{I}^{(2)}\left(u_{1}, \ldots, u_{5}\right)(t):=-i \sum_{\ell=1}^{3} \int_{0}^{t} S\left(t-t^{\prime}\right) v_{j_{1}} \overline{v_{j_{2}}} v_{j_{3}}\left(t^{\prime}\right) d t^{\prime},
$$

where $v_{j_{i}}=u_{i}$ for $i \neq \ell$ and $v_{j_{\ell}}=\mathcal{I}\left(u_{\ell}, u_{4}, u_{5}\right)$. This basically corresponds to the second order term appearing in the power series expansion for (1.1). In particular, we have that $z_{5}=\mathcal{I}^{(2)}\left(z_{1}, \ldots, z_{1}\right)$.

By a computation similar to the proof of Lemma A.1 we can prove the following deterministic non-smoothing on the second order term. Let $\sigma>5 s-2$. Then, there is no finite constant $C>0$ such that

$$
\left\|\mathcal{I}^{(2)}\left(S(t) \phi_{1}, \ldots, S(t) \phi_{5}\right)\right\|_{X^{\sigma}([0,1])} \leq C \prod_{j=1}^{5}\left\|\phi_{j}\right\|_{H^{s}}
$$

for all $\phi_{j} \in H^{s}\left(\mathbb{R}^{3}\right)$. In particular, this shows that when $s \leq \frac{1}{2}$, there is no deterministic smoothing on the second order term $\mathcal{I}^{(2)}$. A similar comment applies to the higher order terms.

\section{REFERENCES}

[1] Árpád Bényi, Tadahiro Oh, and Oana Pocovnicu, Wiener randomization on unbounded domains and an application to almost sure well-posedness of NLS, Excursions in harmonic analysis. Vol. 4, Appl. Numer. Harmon. Anal., Birkhäuser/Springer, Cham, 2015, pp. 3-25. MR 3411090

[2] Árpád Bényi, Tadahiro Oh, and Oana Pocovnicu, On the probabilistic Cauchy theory of the cubic nonlinear Schrödinger equation on $\mathbb{R}^{d}, d \geq 3$, Trans. Amer. Math. Soc. Ser. B 2 (2015), 1-50, DOI 10.1090/btran/6. MR.3350022

[3] Á. Bényi, T. Oh, and O. Pocovnicu, On the probabilistic Cauchy theory for nonlinear dispersive PDEs, Landscapes of Time-Frequency Analysis, 1-32, Appl. Numer. Harmon. Anal., Birkhäuser/Springer, 2019.

[4] J. Bourgain, Fourier transform restriction phenomena for certain lattice subsets and applications to nonlinear evolution equations. I. Schrödinger equations, Geom. Funct. Anal. 3 (1993), no. 2, 107-156, DOI 10.1007/BF01896020. MR1209299

[5] Jean Bourgain, Invariant measures for the 2D-defocusing nonlinear Schrödinger equation, Comm. Math. Phys. 176 (1996), no. 2, 421-445. MR1374420

[6] J. Bourgain, Refinements of Strichartz' inequality and applications to $2 D-N L S$ with critical nonlinearity, Internat. Math. Res. Notices 5 (1998), 253-283, DOI 10.1155/S1073792898000191. MR1616917

[7] J. Brereton, Almost sure local well-posedness for the supercritical quintic NLS, Tunisian J. Math. 1 (2019), no. 3, 427-453.

[8] Nicolas Burq and Nikolay Tzvetkov, Random data Cauchy theory for supercritical wave equations. I. Local theory, Invent. Math. 173 (2008), no. 3, 449-475, DOI 10.1007/s00222-0080124-z. MR2425133

[9] Nicolas Burq and Nikolay Tzvetkov, Probabilistic well-posedness for the cubic wave equation, J. Eur. Math. Soc. (JEMS) 16 (2014), no. 1, 1-30, DOI 10.4171/JEMS/426. MR3141727

[10] Thierry Cazenave and Fred B. Weissler, Some remarks on the nonlinear Schrödinger equation in the critical case, Nonlinear semigroups, partial differential equations and attractors (Washington, DC, 1987), Lecture Notes in Math., vol. 1394, Springer, Berlin, 1989, pp. 18-29, DOI 10.1007/BFb0086749. MR.1021011

[11] M. Christ, Power series solution of a nonlinear Schrödinger equation, Mathematical aspects of nonlinear dispersive equations, Ann. of Math. Stud., vol. 163, Princeton Univ. Press, Princeton, NJ, 2007, pp. 131-155. MR2333210

[12] M. Christ, J. Colliander, and T. Tao, Ill-posedness for nonlinear Schrödinger and wave equations, arXiv:math/0311048 [math.AP]. 
[13] J. Colliander, M. Keel, G. Staffilani, H. Takaoka, and T. Tao, Global existence and scattering for rough solutions of a nonlinear Schrödinger equation on $\mathbb{R}^{3}$, Comm. Pure Appl. Math. 57 (2004), no. 8, 987-1014, DOI 10.1002/cpa.20029. MR2053757

[14] J. Colliander, M. Keel, G. Staffilani, H. Takaoka, and T. Tao, Global well-posedness and scattering for the energy-critical nonlinear Schrödinger equation in $\mathbb{R}^{3}$, Ann. of Math. (2) 167 (2008), no. 3, 767-865, DOI 10.4007/annals.2008.167.767. MR2415387

[15] James Colliander and Tadahiro Oh, Almost sure well-posedness of the cubic nonlinear Schrödinger equation below $L^{2}(\mathbb{T})$, Duke Math. J. 161 (2012), no. 3, 367-414, DOI 10.1215/00127094-1507400. MR2881226

[16] Giuseppe Da Prato and Arnaud Debussche, Two-dimensional Navier-Stokes equations driven by a space-time white noise, J. Funct. Anal. 196 (2002), no. 1, 180-210, DOI 10.1006/jfan.2002.3919. MR.1941997

[17] Giuseppe Da Prato and Arnaud Debussche, Strong solutions to the stochastic quantization equations, Ann. Probab. 31 (2003), no. 4, 1900-1916, DOI 10.1214/aop/1068646370. MR2016604

[18] Benjamin Dodson, Global well-posedness and scattering for the defocusing, cubic nonlinear Schrödinger equation when $n=3$ via a linear-nonlinear decomposition, Discrete Contin. Dyn. Syst. 33 (2013), no. 5, 1905-1926, DOI 10.3934/dcds.2013.33.1905. MR.3002734

[19] Peter K. Friz and Martin Hairer, A course on rough paths: With an introduction to regularity structures, Universitext, Springer, Cham, 2014. MR.3289027

[20] J. Ginibre and G. Velo, Smoothing properties and retarded estimates for some dispersive evolution equations, Comm. Math. Phys. 144 (1992), no. 1, 163-188. MR.1151250

[21] Massimiliano Gubinelli and Nicolas Perkowski, Lectures on singular stochastic PDEs, Ensaios Matemáticos [Mathematical Surveys], vol. 29, Sociedade Brasileira de Matemática, Rio de Janeiro, 2015. MR 3445609

[22] M. Hadac, S. Herr, H. Koch, Well-posedness and scattering for the KP-II equation in a critical space, Ann. Inst. H. Poincaré Anal. Non Linéaire 26 (2009), no. 3, 917-941. Erratum to "Well-posedness and scattering for the KP-II equation in a critical space", Ann. Inst. H. Poincaré Anal. Non Linéaire 27 (2010), no. 3, 971-972. MR2629889

[23] Martin Hairer, Introduction to regularity structures, Braz. J. Probab. Stat. 29 (2015), no. 2, 175-210, DOI 10.1214/14-BJPS241. MR3336866

[24] Sebastian Herr, Daniel Tataru, and Nikolay Tzvetkov, Global well-posedness of the energycritical nonlinear Schrödinger equation with small initial data in $H^{1}\left(\mathbb{T}^{3}\right)$, Duke Math. J. 159 (2011), no. 2, 329-349, DOI 10.1215/00127094-1415889. MR2824485

[25] Hiroyuki Hirayama and Mamoru Okamoto, Random data Cauchy problem for the nonlinear Schrödinger equation with derivative nonlinearity, Discrete Contin. Dyn. Syst. 36 (2016), no. 12, 6943-6974, DOI 10.3934/dcds.2016102. MR3567827

[26] Justin Holmer and Svetlana Roudenko, A sharp condition for scattering of the radial $3 D$ cubic nonlinear Schrödinger equation, Comm. Math. Phys. 282 (2008), no. 2, 435-467, DOI 10.1007/s00220-008-0529-y. MR2421484

[27] Jean-Pierre Kahane, Some random series of functions, 2nd ed., Cambridge Studies in Advanced Mathematics, vol. 5, Cambridge University Press, Cambridge, 1985. MR.833073

[28] Markus Keel and Terence Tao, Endpoint Strichartz estimates, Amer. J. Math. 120 (1998), no. 5, 955-980. MR 1646048

[29] Carlos E. Kenig and Frank Merle, Scattering for $\dot{H}^{1 / 2}$ bounded solutions to the cubic, defocusing NLS in 3 dimensions, Trans. Amer. Math. Soc. 362 (2010), no. 4, 1937-1962, DOI 10.1090/S0002-9947-09-04722-9. MR2574882

[30] R. Killip, J. Murphy, and M. Visan, Almost sure scattering for the energy-critical NLS with radial data below $H^{1}\left(\mathbb{R}^{4}\right)$, arXiv:1707.09051 [math.AP], 2017.

[31] Herbert Koch and Daniel Tataru, A priori bounds for the $1 D$ cubic NLS in negative Sobolev spaces, Int. Math. Res. Not. IMRN 16 (2007), Art. ID rnm053, 36, DOI 10.1093/imrn/rnm053. MR2353092

[32] Jonas Lührmann and Dana Mendelson, Random data Cauchy theory for nonlinear wave equations of power-type on $\mathbb{R}^{3}$, Comm. Partial Differential Equations 39 (2014), no. 12, 2262-2283, DOI 10.1080/03605302.2014.933239. MR3259556

[33] Jonas Lührmann and Dana Mendelson, On the almost sure global well-posedness of energy sub-critical nonlinear wave equations on $\mathbb{R}^{3}$, New York J. Math. 22 (2016), 209-227. MR 3484682 
[34] H. P. McKean, Statistical mechanics of nonlinear wave equations. IV. Cubic Schrödinger, Comm. Math. Phys. 168 (1995), no. 3, 479-491. MR1328250

[35] Andrea R. Nahmod and Gigliola Staffilani, Almost sure well-posedness for the periodic 3D quintic nonlinear Schrödinger equation below the energy space, J. Eur. Math. Soc. (JEMS) 17 (2015), no. 7, 1687-1759, DOI 10.4171/JEMS/543. MR3361727

[36] Tadahiro Oh, A remark on norm inflation with general initial data for the cubic nonlinear Schrödinger equations in negative Sobolev spaces, Funkcial. Ekvac. 60 (2017), no. 2, 259-277. MR.3702002

[37] T. Oh, M. Okamoto, and O. Pocovnicu, On the probabilistic well-posedness of the nonlinear Schrödinger equations with non-algebraic nonlinearities, arXiv:1708.01568 [math.AP], Discrete Contin. Dyn. Syst. A., to appear.

[38] Tadahiro Oh and Oana Pocovnicu, Probabilistic global well-posedness of the energycritical defocusing quintic nonlinear wave equation on $\mathbb{R}^{3}$ (English, with English and French summaries), J. Math. Pures Appl. (9) 105 (2016), no. 3, 342-366, DOI 10.1016/j.matpur.2015.11.003. MR3465807

[39] T. Oh, O. Pocovnicu, and Y. Wang, On the stochastic nonlinear Schrödinger equations with non-smooth additive noise, arXiv:1805.08412 [math.AP], Kyoto J. Math, to appear.

[40] T. Oh, N. Tzvetkov, and Y. Wang, Solving the $4 N L S$ with white noise initial data, preprint.

[41] T. Ozawa and Y. Tsutsumi, Space-time estimates for null gauge forms and nonlinear Schrödinger equations, Differential Integral Equations 11 (1998), no. 2, 201-222. MR1741843

[42] R. E. A. C. Paley and A. Zygmund, On some series of functions (1), (2), (3), Proc. Camb. Philos. Soc. 26 (1930), 337-357, 458-474; 28 (1932), 190-205.

[43] Oana Pocovnicu, Almost sure global well-posedness for the energy-critical defocusing nonlinear wave equation on $\mathbb{R}^{d}, d=4$ and 5, J. Eur. Math. Soc. (JEMS) 19 (2017), no. 8, 2521-2575, DOI 10.4171/JEMS/723. MR 3668066

[44] Oana Pocovnicu and Yuzhao Wang, An $L^{p}$-theory for almost sure local well-posedness of the nonlinear Schrödinger equations (English, with English and French summaries), C. R. Math. Acad. Sci. Paris 356 (2018), no. 6, 637-643, DOI 10.1016/j.crma.2018.04.009. MR3806892

[45] Aurélien Poiret, Didier Robert, and Laurent Thomann, Probabilistic global well-posedness for the supercritical nonlinear harmonic oscillator, Anal. PDE 7 (2014), no. 4, 997-1026, DOI 10.2140/apde.2014.7.997. MR3254351

[46] Robert S. Strichartz, Restrictions of Fourier transforms to quadratic surfaces and decay of solutions of wave equations, Duke Math. J. 44 (1977), no. 3, 705-714. MR0512086

[47] Laurent Thomann, Random data Cauchy problem for supercritical Schrödinger equations (English, with English and French summaries), Ann. Inst. H. Poincaré Anal. Non Linéaire 26 (2009), no. 6, 2385-2402, DOI 10.1016/j.anihpc.2009.06.001. MR2569900

[48] Norbert Wiener, Tauberian theorems, Ann. of Math. (2) 33 (1932), no. 1, 1-100, DOI 10.2307/1968102. MR.1503035

[49] Kenji Yajima, Existence of solutions for Schrödinger evolution equations, Comm. Math. Phys. 110 (1987), no. 3, 415-426. MR891945

[50] Ting Zhang and Daoyuan Fang, Random data Cauchy theory for the generalized incompressible Navier-Stokes equations, J. Math. Fluid Mech. 14 (2012), no. 2, 311-324, DOI 10.1007/s00021-011-0069-7. MR2925111

Department of Mathematics, Western Washington University, 516 High Street, BelLINGHAM, WASHINGTON 98225

Email address: arpad.benyi@wwu.edu

School of Mathematics, The University of Edinburgh, and The Maxwell Institute for the Mathematical Sciences, James Clerk Maxwell Building, The King's Buildings, Peter Guthrie Tait Road, Edinburgh, EH9 3FD, United Kingdom

Email address: hiro.oh@ed.ac.uk

Department of Mathematics, Heriot-Watt University, and The Maxwell Institute For the Mathematical Sciences, Edinburgh, EH14 4AS, United Kingdom

Email address: o.pocovnicu@hw.ac.uk 